\title{
Empfehlungen für
}

\section{integrationspolitische Best-Practice-}

\section{Strategien und -Maßnahmen}

Basierend auf der Studie LODA: LoslassenDurchstehen-Ankommen: Eine transdisziplinäre Studie zur rezenten Situation Geflüchteter in Österreich

Sabine Bauer-Amin, Josef Kohlbacher, Marie Lehner, Gabriele RasulyPaleczek, Leonardo Schiocchet, Maria Six-Hohenbalken (Hg.) 
Copyrighted Material

Empfehlungen für integrationspolitische Best-Practice-Strategien und -Maßnahmen

Copyright (C 2020 by Sabine Bauer-Amin, Josef Kohlbacher, Marie Lehner, Gabriele Rasuly-Paleczek, Leonardo Schiocchet, and MariaAnna Six-Hohenbalken / ROR-n Plattform / ROR-n. All Rights Reserved.

\section{D.O.I. https://doi.org/10.1553/RoR-n Plattform Vol 01(1)}

ISSN: 2707-8760 (online) ; 2707-8752 (print)

No part of this publication may be reproduced, stored in a retrieval system or transmitted in any form or by any means, electronic, mechanical, photocopying, recording or otherwise, without prior permission of the copyright holder, except for the inclusion of quotations properly cited.

Published in Vienna, Austria by ROR-n, with support from: Institut für Stadt- und Regionalforschung (ÖAW); Institut für Sozialanthropologie (ÖAW); in cooperation with the Institut für Kultur-und Sozialanthropologie, Universität Wien.

Financed by the Innovationsfonds, ÖAW

\section{\begin{tabular}{l|l} 
Refugee Outreach & $\mathrm{R} O \mathrm{R}-\boldsymbol{n}$
\end{tabular}}

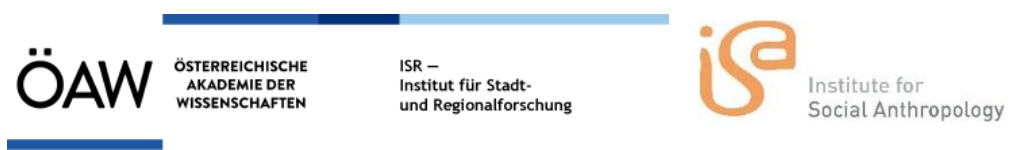


Index

Einleitende Bemerkungen

Josef Kohlbacher.

5

Empfehlungen basierend auf der Teilstudie mit

Respondent/inn/en aus Afghanistan

Josef Kohlbacher

Empfehlungen basierend auf der Teilstudie mit Respondent/inn/en aus Syrien und dem Irak in Wien

Sabine Bauer-Amin................................................. 76

Wellbeing and Psychological Distress among Refugees of the Syrian and Iraqi Conflicts

Leonardo Schiocchet. 105

Integration von Geflüchteten in der Gemeinde St. AndräWördern (NÖ)

Andreas Schwarzbauer.

Integration von Geflüchteten in der Gemeinde und im Bezirk Oberwart (B)

Hannah Lichtenwagner.

Integration von Asylwerber/inne/ $\mathrm{n}$ und Asylberechtigten in den Arbeitsmarkt. Politik und Maßnahmen in Oberösterreich 
Integration von Geflüchteten in den Bildungssektor

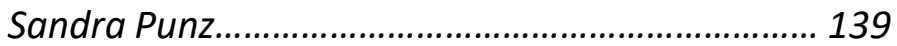

Diasporische Gemeinschaften und ihr zivilgesellschaftliches Engagement

Maria Six-Hohenbalken...................................... 148

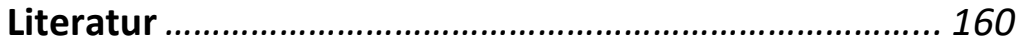





\section{Einleitende Bemerkungen}

\section{Josef Kohlbacher}

Die Flucht- und Flüchtlingsforschung ist ausgeprägt interdisziplinär und benutzt eine Vielfalt an methodischen und theoretischen Zugängen. Des Weiteren hat sie sich in den vergangenen Jahren auch immer stärker ausdifferenziert, wendet sich grundsätzlich gegen Kategorisierungen und hebt die Individualität und Heterogenität der Geflüchteten hervor. Diese unterscheiden sich wesentlich von Arbeitsmigrant/inn/en, da ihre Mobilität auf keiner freien Entscheidung basiert und sie sich erst um die Wiederherstellung sozialer und ökonomischer Rahmenbedingungen bemühen müssen. Geflüchtete Menschen aus den drei Herkunftsstaaten Afghanistan ${ }^{1}$, Syrien und Irak, deren Erfahrungen, Ziele, Strategien und Anliegen, vor allem aber deren soziale und Systemintegration werden von uns als Ausgangsbasis für die vorgeschlagenen Best-Practice-Maßnahmen im Rahmen der folgenden Zusammenstellung in den Fokus gestellt.

Ein wesentliches Ziel des Projekts LODA (Loslassen Durchstehen - Ankommen) lag neben der Akquisition neuer wissenschaftlicher Daten über die nach Österreich Geflüchteten in der handlungsrelevanten Umsetzung der Resultate und in weiterer Folge in der Formulierung

${ }^{1}$ Ein Teil der befragten Afghan/inn/en stammte aus dem Iran bzw. aus Pakistan, wohin entweder sie selbst oder ihre Familien im Verlauf der vergangenen Jahrzehnte geflüchtet waren. 
konkreter Handlungsempfehlungen. Um die nach wie vor bestehenden Practice-Defizite auszugleichen und die vielfältigen Potenziale der geflüchteten Menschen besser erschließen zu können, sind ungeachtet der seit Projektbeginn erheblich zurückgegangenen Zahlen an Asylwerber/inne/n weiterhin gezielte Integrationsmaßnahmen vonnöten.

Im Rahmen des Forschungsdesigns wurde ein Bottom-up-Ansatz gewählt, sodass die unmittelbar Betroffenen, also Expert/inn/en aus der Praxis der Flüchtlingsarbeit und vor allem die Geflüchteten selbst, nach ihren Vorschlägen für Verbesserungen hinsichtlich der Entscheidungsgrundlagen und der Performance der politischen und praktischen Maßnahmen im Umgang mit der Integration der Geflüchteten gefragt wurden. Deutlich spiegelten sich in unserem Interviewsample die Komplexität der Hintergründe, Rahmenbedingungen und Auswirkungen von Fluchtbewegungen sowie die vielfältigen daraus resultierenden integrationspolitischen Konsequenzen wider. Konsens besteht seitens der Autor/inn/en darüber, dass es sinnvoll ist, bei allen Geflüchteten Integrationsbemühungen so früh wie möglich zu setzen, also bereits bei Asylwerber/inne/n im laufenden Verfahren, wenngleich bei diesen noch nicht entschieden ist, ob der betreffenden Person dauerhaft Aufenthalt in Österreich zuerkannt wird.

Die Thematik der Flucht berührt eine breite Palette an Politikfeldern und Systemen, die räumlich von der kommunalen (munizipalen) über die föderale und bis zur nationalen Ebene reichen, auf sehr divergente Art und Weise. Die äußerst vielschichtige Thematik wird allerdings hinsichtlich der politischen Entscheidungsfindungsprozesse, aber auch der 
institutionellen Maßnahmenkonzipierung in den Medien sowie im politischen Diskurs häufig unausgewogen und in unzureichend reflektierter Weise aufgegriffen. Daraus resultieren nicht selten diskriminierende Zuschreibungen und vorurteilsbehaftete Stereotypisierungen, von denen in unseren Interviews nicht selten berichtet wurde, und gegen die wir mit einigen unseren Best-PracticeVorschläge auch Stellung beziehen möchten.

Die zum Zeitpunkt unserer Erhebungen dominierenden Rahmenbedingungen ließen Defizite in den auf unterschiedlichste Aspekte der Integration bezogenen Maßnahmen erkennen. Es bestand also dringender Handlungsbedarf, auf welchen die befragten Expert/inn/en mit zahlreichen Empfehlungen an das Forscher/innenteam hingewiesen haben.

Die Datenakquise war infolge des Sampleumfangs, der muttersprachlichen Interviews, der erforderlichen Übersetzungen sowie Transkripte des narrativen Materials für die Forscher/innen ein überaus zeitaufwendiger Prozess, was auch eine Projektlaufzeit von zwei Jahren unbedingt nötig machte. Seit der Projektantragstellung bzw. dem Start der empirischen Erhebungen zum Projekt LODA haben sowohl auf der Bundes- als auch den Länderebenen in Österreich grundlegende politische Umstrukturierungen und Paradigmenwechsel sowie Reformen vieler integrationsbezogener Maßnahmen stattgefunden, die auch zu Veränderungen im Umgang mit den Geflüchteten sowie in den institutionellen Abläufen führten. Einige der seitens der Expert/inn/en sowie der Geflüchteten klassifizierte Problemkonstellationen haben sich seit der "refugee crisis" sowie innerhalb der zweijährigen Projektlaufzeit deutlich modifiziert: 
Manche haben sich schon allein infolge der Rückgänge bei den Asylanträgen abgemildert (z.B. die vielfach kritisierte Unterbringung in Massenquartieren), dazu kamen die professionellere Organisation und Adaption der Inhalte der Kompetenzchecks, Veränderungen der Beratungsinfrastruktur des AMS sowie bei den Deutschkursen. Andere Herausforderungen sind im Rahmen der nun dauerhaften Integration der Geflüchteten auf den regionalen Arbeits- und Wohnungsmärkten hinzugekommen oder wurden verstärkt (z.B. Probleme der Wohnintegration infolge einer allgemeinen Verknappung von Wohnraum in den Metropolen, Konzentrationen arbeitssuchender Asylberechtigter in den urbanen Räumen). Auch der politische und mediale Diskurs hat sich nicht nur in Österreich, sondern in allen EU-Staaten seit 2015/16 fundamental verändert, was sich auch in den Alltagserfahrungen vieler Geflüchteter widerspiegelte.

Das Zusammenspiel all dieser Prozesse hatte zur Folge, dass das empirische Material aus der Befragung nun als zum Teil etwas veraltet zu bezeichnen ist. Das Autor/inn/enteam war daher für die Erstellung dieses Papers mit einer in den Sozialwissenschaften eher seltenen Herausforderung konfrontiert, dass nämlich die empirischen Daten im historischen Kontext des Erhebungszeitraums zu interpretieren sind. Aufgrund der geänderten Konstellationen haben wir innerhalb des Projektteams auf konsensuale Weise auch den Entschluss gefasst, den im Projektantrag als „Policy Recommendations" bezeichneten handlungsrelevanten Output durch Vorschläge und Konzepte für konstruktive Best-Practice-Empfehlungen zu ersetzen.

Die Grundstruktur des vorliegenden Best-PracticePapers folgt der Aufgliederung nach relevanten 
Intergrationsfeldern - sowohl der Systemintegration wie auch der Sozialintegration (vgl. Lockwood 1964, Esser 2001), darüber hinaus finden Querschnittsmaterien, wie etwa die Wichtigkeit realistischer Perspektiven, Antidiskriminierungsstrategien, Vermittlung von Systemwissen, die Bedeutung vielfältiger aufeinander abgestimmter Kooperationen zwischen relevanten Organisationen und Entscheidungsinstanzen, die Bedeutung des Engagements von Ehrenamtlichen etc., entsprechend Berücksichtigung. Im Folgenden werden nach Integrationsbereichen untergliedert und basierend auf den empirischen Resultaten - die strategischen und Handlungsempfehlungen für die bestmögliche Fortsetzung bzw. Modifikation bestehender und die Installierung neuer Maßnahmen zur Integrationsförderung in den relevanten Feldern vorgestellt. Die empirische Basis bilden 14 leitfadengestützte Expert/inn/en- und in summa 91 Respondent/inn/eninterviews (46 mit Geflüchteten aus Syrien, 11 mit Iraker/inne/n und 34 mit geflüchteten Afghan/inn/en).

Im Rahmen der kompilierten Best-PracticeEmpfehlungen stehen bestimmte Felder (vor allem der Systemintegration) im Vordergrund, während andere Integrationsbereiche weniger Berücksichtigung finden. Wir haben uns diesbezüglich an unserem empirischen Material orientiert, in dem klare Schwerpunkte erkennbar waren, während andere Integrationsbereiche seitens der Befragten weit seltener Erwähnung fanden. Zahlenmäßig im Vordergrund standen Empfehlungen in Bezug auf das Asylverfahren, die berufliche Integration, den Zugang zu Bildung und damit im Zusammenhang stehende Aspekte, des Weiteren die Wohnintegration 
und Erfahrungen im Gesundheitswesen. In vielen Zusammenhängen kamen die negativen Auswirkungen der langen Dauer der Asylanerkennungsverfahren zur Sprache. Weitere in unterschiedlichen Kontexten angesprochene Aspekte bezogen sich auf Verbesserungen bei der Vernetzung von Institutionen und Organisationen, die Informationsbereitstellung für unterschiedliche Rezipient/inn/engruppen, Defizite in der Informationsdiffusion, Hürden im Zugang zu relevanten Informationen, den Bedarf an Antidiskriminierungsmaßnahmen sowie eine generelle Objektivierung der fluchtbezogenen Diskurse.

Sehr zahlreich waren die Empfehlungen im Zusammenhang mit dem Spracherwerb, z.B. bezogen auf möglichst frühzeitige, intensive Unterstützung und die Gewährung von Folgekursen, den Zugang zu weiterführender Schulbildung, berufliche Höherqualifizierung etc. Des Weiteren wurde auch eine stärker auf individuelle Bedürfnisse abgestimmte Zuerkennung von Bildungs-, Arbeits- und Mobilitätsrechten gefordert.

Abschließend ist festzustellen, dass es weiterer empirischer Analysen zu Entscheidungsprozessen und zur Implementierung von Maßnahmen im internationalen Flüchtlingsregime bedarf. Dabei sollten verstärkt Non-Governmental-Akteur/inn/e/n, vor allem die Geflüchteten selbst, die ethnischen Communities und diverse privat Engagierte, wie etwa Ehrenamtliche, berücksichtigt werden.

Wir verwenden im Folgenden den im wissenschaftlichen Diskurs häufig kritisierten Terminus der „Integration“, obwohl wir uns dessen Problematik und der teils sehr unterschiedlichen Definitionen völlig bewusst sind. „Integration“ und "Inklusion" sind zwei 
Begriffe, die im Zusammenhang mit Migration häufig als Auslöser von ideologisch fundierten, kontroversiellen Debatten fungieren. Nicht selten werden sie synonym benutzt, viele Wissenschaftler/innen wie auch Praktiker/innen orten allerdings erhebliche Differenzen. Da es sich bei dem vorliegenden Papier aber um eine praxisorientierte Kompilation von Handlungsempfehlungen handelt, die ihrer Grundstruktur nach den Feldern der System- und Sozialintegration folgt, halten wir die Verwendung eines weit gefassten und kritisch angewendeten Integrationsbegriffs hier für vertretbar. 


\title{
Empfehlungen basierend auf der Teilstudie mit Respondent/inn/en aus Afghanistan
}

\author{
Josef Kohlbacher
}

\section{A INTEGRATIONSBEREICHEÜBERGREIFENDE BEST- PRACTICE-VORSCHLÄGE}

Die im Folgenden präsentierten Vorschläge beziehen sich nicht exklusiv auf einen bestimmten Integrationsbereich, sondern repräsentieren jeweils übergreifende Handlungsempfehlungen.

Integration als idealtypische Querschnittsmaterie betrifft alle Ebenen gesellschaftlichen Zusammenlebens, wie Arbeiten, Wohnen, Bildung, soziale Interaktionen, interethnisches Zusammenleben, Konfliktprävention usw.

- Geflüchtete sind Menschen mit vielfältigen Wünschen, Bedürfnissen und Fähigkeiten und daher wird von Expert/inn/enseite für eine stärkere Individualisierung bestehender sowie die Installierung neuer Maßnahmen plädiert, welche ein spezifischeres Eingehen auf das jeweilige Individuum und dessen Bedürfnisse ermöglichen sollen. Wir halten es primär für wichtig, die Eigenverantwortlichkeit von geflüchteten Männern und Frauen zu stärken. Zur Erreichung dieses Ziels sollten Rechte, Wünsche und Fähigkeiten der Menschen bei der Formulierung und Gestaltung von 
Integrationsmaßnahmen generell stärker berücksichtigt werden. Die Forcierung vorhandener persönlicher Potentiale sowie die Berücksichtigung von individuellen Präferenzen stärkt die Eigenverantwortung, macht unabhängiger von Zuwendungen der Aufnahmegesellschaft (ein in der öffentlichen Diskussion häufig geäußerter Vorwurf) und führt insgesamt zu mehr Empowerment. Hierbei ist insbesondere auch auf das Empowerment von geflüchteten Frauen Augenmerk zu legen.

- Zusätzlich zu diesem Individualansatz erscheint uns aber auch wichtig, einen möglichst ganzheitlichen Blick auf die Anforderungen für Geflüchtete in den Bereichen der Sozial- und Systemintegration und vor allem auf deren vielfältige Wechselwirkungen zu gewinnen.

- Hervorzuheben ist auch, dass die Schaffung und Gewährleistung von dauerhaften und realistischen Perspektiven von eminenter Wichtigkeit für den individuellen Integrationsverlauf ist. Aufenthaltsperspektiven sind eine Voraussetzung zum Beispiel auch für die Orientierung an und das Erreichen von Bildungszielen. Bei älteren Geflüchteten, aber nicht selten auch bei interviewten Frauen sind die Möglichkeiten infolge von arbeitsmarktabhängigen oder familiären Rahmenbedingungen stärker eingeengt. Diesem Personenkreis kann eine Perspektivenverlagerung in die nächste Generation, in Bildungs- und Aufstiegschancen für die Kinder, helfen, eine gewisse 
Zukunftsorientierung und Motivationen zu sichern.

- Generell fehlt, wie einige Expert/inn/en anmerkten, gesichertes, mit qualitativen Methoden erhobenes empirisches Datenmaterial darüber, wie die Angebote in den diversen Integrationsbereichen bei den Geflüchteten tatsächlich ankommen, wie sie von diesen rezipiert werden und inwieweit sie daraus für ihre eigene Bildungs- und Berufsbiographie konkreten Mehrwert ziehen können. In diesem Bereich der längerfristigen Wirkungsforschung orten wir ein wichtiges Forschungsdesiderat für die nahe und mittelfristige Zukunft der interdisziplinären sozialwissenschaftlichen Forschung zur Integration von Geflüchteten.

- Zweifellos ist ein erhebliches Mehr an Realitätsbezug hinsichtlich der Erwartungen (auf beiden Seiten) vonnöten. Geflüchtete können nicht mit den Maßstäben von Arbeitsmigrant/inn/en gemessen werden, die sich bewusst und in geordneten Rahmenbedingungen für Mobilität entschieden haben, um ihre Kompetenzen auf dem Arbeitsmarkt des Ziellandes einzusetzen. Nicht wenige Menschen mit Fluchterfahrungen müssen Traumata verarbeiten, sie sind mit dem eigenen unsicheren Status oder dem ihrer Familie konfrontiert, müssen sich erst die im Aufnahmeland umsetzbaren Qualifikationen erwerben und sind folglich nur mittelfristig als vollwertige Arbeitskräfte einsetzbar.

- Grundsätzlich spiegelt unser Material die institutionell-legistisch bedingten Zäsuren bzw. 
Übergänge und die damit zusammenhängenden Hürden in der Individualbiographie als besonders herausfordernd wider. Ein markantes Beispiel ist die viermonatige Phase $\mathrm{NACH}$ Erhalt des Asylbescheids binnen welcher der Auszug aus dem Erstversorgungsquartier zu erfolgen hat. Ein weiteres Beispiel für eine schwierige Übergangsphase ist jene von UMFs mit Eintritt der Volljährigkeit. Zwar standen UMFs nicht im Fokus unserer Erhebung, die Notwendigkeit zusätzlicher Beratungs- und Hilfeleistungen im Übergang zum selbständigen Leben als Erwachsener wurde von Expert/inn/enseite aber mehrmals problematisiert.

- Ein durchgängig wichtiger Aspekt scheint uns in der Hervorhebung von positiven Role Models erfolgreicher Personen mit Fluchtbiographien als Orientierungsinstanzen zu liegen.

- Von Expert/inn/enseite hervorgehoben wurde auch die immense Wichtigkeit von Mentor/inn/en bzw. Buddies als Informant/inn/en und Begleiter/innen in allen integrationsrelevanten Bereichen, vor allem aber im Feld der sozialen Integration (vgl. entsprechendes Subkapitel).

- Eine weitere Querschnittsmaterie bezieht sich auf die Vermittlung von Systemwissen, welches den Rahmen der aktuellen Curricula der Wertekurse sprengen würde. Die grundlegenden Systeme Arbeits- und Wohnungsmarkt, Bildung, Gesundheit, Recht etc. sind hinsichtlich ihrer Funktionen, interner Abläufe und Entscheidungsprozesse für viele Geflüchtete nur schwer verständlich, unterscheiden sie sich doch 
erheblich von jenen in den Herkunftsländern. Hier wäre die Installierung systematisierter Maßnahmen zur Information und Explikation der wichtigsten Strukturen, Funktionsmechanismen und Abläufe der relevanten Systeme in Österreich für ein besseres Verständnis ratsam. Die Wertekurse leisten hierzu einen konstruktiven Beitrag, können angesichts ihres beschränkten zeitlichen Rahmens aber nicht alle Informationsdefizite abdecken.

- Viele Expert/inn/en plädieren zur Vermeidung von Ineffizienzen für eine Verbesserung organisatorischer Abläufe sowie für ein Mehr an Koordination innerhalb und zwischen den in die Arbeit mit Geflüchteten involvierten Organisationen und Institutionen. Damit in einem Konnex steht auch die Empfehlung, bestehende Kommunikationsdefizite zu minimieren und auf institutioneller Ebene möglichst eng zusammenzuarbeiten.

- Sowohl seitens der Expert/inn/en als auch vieler Geflüchteter wird eine Versachlichung und Entemotionalisierung der Diskurse und stigmatisierenden Medienberichterstattung im Sinne der Reduktion bzw. Verhinderung von Vorurteilen gefordert. Eine Verstärkung des antirassistischen Diskurses sowie übergreifende mediale Strategien gegen Vorurteile wären in diesem Zusammenhang als Ziele zu formulieren. In diese Richtung geht auch die Empfehlung der Versachlichung und Entemotionalisierung der Diskurse über Geflüchtete generell.

- Etliche Befragte haben diskriminierende Erfahrungen im Umgang mit Ämtern und 
Behörden oder in Deutschkursen machen müssen, wurden ihrer mangelnden Sprachkenntnisse wegen kritisiert. Im Zusammenhang damit werden zusätzliche Antidiskriminierungsmaßnahmen und -gesetze, Schulungen der Mitarbeiter/innen etwa bei Behörden empfohlen. Auch das Tragen des Kopftuchs wird als eine Ursache für vielfältige Alltagsdiskriminierungen erlebt und in diesem Zusammenhang haben die betroffenen Respondentinnen Erwartungen verbalisiert, dass die Politik hier Ausgleich schaffen sollte.

- Die Schaffung eines generell integrationsfreundlichen Klimas würde wesentlich zum individuellen Wohlbefinden der Geflüchteten beitragen. Voraussetzungen hierfür sind u.a die Förderung von interkulturellem Verständnis und die Reduktion von Vorurteilen.

- Eine weitere generelle Forderung bezieht sich auf die verstärkte Involvierung von Fachleuten und Wissenschaftler/inne/n in Entscheidungsfindungsprozesse und in die Konzipierung von Maßnahmen. Auch Expert/inn/en aus den ethnischen Communities sollten in Beratungs- und Organisationsprozesse involviert werden, um aus ihrem Kontextwissen entsprechenden Mehrwert zu ziehen. Allerdings ist anzumerken, dass Angehörige der Communities nicht quasi automatisch Expert/inn/enstatus besitzen. Nicht wenige fühlen sich durch die an sie herangetragenen, heterogenen Erwartungen (einerseits der Geflüchteten aus den eigenen Communities und 
andererseits der österreichischen Aufnahmegesellschaft und deren Institutionen) überfordert. Regelmäßiges Coaching - dies gilt auch für Mitarbeiter/innen von NGOs und in der Flüchtlingshilfe generell - muss dringend empfohlen werden.

Eine breite Palette von Desideraten sowohl von Expert/inn/en als auch der Geflüchteten bezog sich auf bestehende Informationsdefizite und den Bedarf an Informationsangeboten jeglicher Form in den verschiedensten Integrationsfeldern. Hier stellt sich als generelle Frage, wie man den Transfer und die Kommunikation des nun bereits viel reichlicher als zum Zeitpunkt der Projektantragstellung vorhandenen Informationsmaterials (z.B. Broschüren, Apps) zu den Geflüchteten als dessen Rezipient/inn/en bewerkstelligen kann. Aus kulturanthropologischer Sicht ist wichtig anzumerken, dass viele Geflüchtete aus bildungsfernen Schichten und somit aus keiner Lesekultur stammen. Vor allem in Afghanistan kommt oraler Informationsvermittlung bis dato noch immer ein hoher Stellenwert zu.

Handlungs- und maßnahmenbezogene Empfehlungen

- beziehen sich auf eine Anhebung und Verbesserung des Informationsstandes der Geflüchteten sowie der österreichischen Bevölkerung über die Situation und die Strukturen in den Herkunftsländern,

- ein möglichst breites und diversifiziertes Informationsangebot sowie Informationsveranstaltungen besonders im ländlichen Raum, in den kleineren Gemeinden, aber 
auch für die Mitarbeiter/innen in der Arbeit mit Geflüchteten,

- die Informationsdiffusion durch Verstärkung von Medienarbeit,

- gebündelte und diversifizierte Informationsangebote, welche Möglichkeiten Geflüchteten in den jeweiligen Phasen, in denen sie sich gerade befinden, offenstehen,

- die Ausweitung des spezifischen Informationsangebots für Frauen zur Wahrnehmung ihrer Rechte.

- sowie die Verbesserung des Informations- und Wissenstransfers zwischen bereits bestehenden Systemen und Praktiken.

- Wir schlagen generell vor, weniger schriftliches Informationsmaterial zum Einsatz zu bringen als vielmehr z.B. YouTube-Filme oder diverses Videomaterial.

\section{Beschreibung der InformantInnen - Abkürzungen}

Im Zeitraum vom 04.08.2017 bis 15.01.2018 wurde ein Sample von 14 Expert/inn/eninterviews akkumuliert. Bei einem Teil davon handelte es sich um Teilnehmer/innen der LODA-Kick-off-Konferenz im Mai 2017. Sie deckten ein äußerst breites Spektrum an Organisationen und Institutionen und somit vielfältige Expertisen zu verschiedensten Bereichen der Arbeit mit und der Integration von Geflüchteten ab. Unter anderem waren die folgenden Organisationen vertreten: Wirtschaftskammer Österreich (WKO), Österreichischer Integrationsfonds (ÖIF), Arbeitsmarktservice Wien (AMS), Magistrat der Stadt Wien - MA 17, Fonds Soziales Wien, Bildungsdirektion für Wien 
(ehem. Stadtschulrat), Diakonie Flüchtlingsdienst, Frauenberatung Wien - Diakonie, Integrationshaus, Studentpoint - Uni Wien, Flüchtlinge Willkommen u.a. NGOs. Nahezu alle Expert/inn/en haben sich die Anonymisierung ihrer Stellungnahmen ausbedungen, weshalb im Folgenden mittels des Kürzels "EXP" Interviews mit Expert/inn/en ohne Namensnennung explizit ausgewiesen werden. Die Interviews mit den afghanischen Respondent/inn/en werden mittels des Kürzels "RESP" kenntlich gemacht.

\section{Zitate aus den Interviews:}

„R: Und da find'ich den Punkt voll wichtig, dass man z.B. in den Gemeinden so Infoveranstaltungen macht. Wo kommen die Leute her? Wie schaut es da aus? Wie sind die aufgewachsen? Wie und wo sind die in die Schule gegangen? Was haben die gelernt, wo sie aufgewachsen sind? Damit man sich einfach besser vorstellen kann, warum die so handeln. ... Das ist aber auch echt so, weil die wirklich dörflichen Bewohner dort, z.B. in der Gemeinde, sich einfach überhaupt nicht vorstellen können wie ein Leben in Afghanistan z.B. ist. Die haben keinen Plan, erstens wie es dort ausschaut. Ich glaub', die könnten es auf der Landkarte nicht einmal ganz genau zeigen alle. Die haben kein Gespür dafür, wie so soziale und kulturelle Strukturen ablaufen. Dass da auch wirklich ein Unterschied ist. Das muss man einfach ganz klar so sagen. Und das können die sich nicht vorstellen, und da tun sie sich auch schwer sich da reinzudenken, weil die Info kriegen sie von nirgends. ... Am Land auch. Und ich glaub' z.B., da würden auch die klassischen FPÖ-Wähler 
hingehen. Weil die interessiert es ja auch voll. 'Wo kommen die her? Und wie leben die?' Das soll ja eigentlich eine Info für alle sein. ... Ganz wichtig finde ich auch diese Länder- bzw. Kulturinfos. Dann find ich einfach ganz wichtig, dass man wegkommt von diesem Anpassungsgedanken, sondern sich überlegt: Was ist Sozialisierung?" (EXP MR6)

„R: ... Oder das Thema Mülltrennung. Das war ganz schwierig und ein Riesenchaos, weil sie den Müll nicht getrennt haben. Da waren Riesenhausstreit. ... Dann hab'ich ihnen einfach irgendwann ein System auf's Aug gedrückt und gesagt: 'So machen wir es jetzt!' und einfach auch eine gewisse Strenge an den Tag gelegt. ... Das hat aber dann funktioniert, weil es den Konflikt gelöst hat." (EXP MR6)

„R: Ich glaub' eine ganz große Herausforderung ist, dass es eigentlich kein gebündeltes Informationsangebot gibt. ... Und das sehen wir als extreme Schwachstelle und mit der Fragestellung beschäftigen wir uns gerade intensiv. Es gibt unterschiedlichste Angebote, sehr viel Gott sei Dank, aber es gibt kein Angebot, wenn man jetzt mal den Perspektivenwechsel betreibt: 'Ich komme nach Österreich, habe einen positiven Asylbescheid und wie geht es jetzt weiter? Ich hab'vielleicht schon ein bisschen was im Herkunftsland gemacht, kann ich mir da Qualifikationen anrechnen lassen? Wie schaut es aus mit Lehrabschluss? Oder in Richtung Schule, Universität?' Also es gibt keinen Verlauf, dass man sagt: 'Okay, ich bin jetzt in der Phase. Welche Möglichkeiten hab'ich? Welches Unterstützungsangebot gibt es?' das fehlt aus meiner Sicht." (EXP MR7) 
„R: Es ist viel zu wenig Unterstützung und hat null Komma null funktioniert. Wie gesagt, eine Stunde pro Woche für 25 Personen. Teilweise Falschinformationen von den NGO-Betreuer/inne/n an die Flüchtlinge. Wirklich null Unterstützung. Also wenn ich jetzt nicht gewesen wäre und andere freiwillige Helfer, dann wäre das im absoluten Chaos ausgeartet. Das ist echt richtig arg, aber da muss Unterstützung vom Land oder vom Staat her. Ich weiß jetzt nicht genau, wer das zahlt, aber ich glaub' das Land Oberösterreich. Das ist wirklich unvorstellbar, wie schlecht das organisiert ist und wie viel Geld die dafür kriegen für das, dass sie nichts machen und total unqualifizierte Leute haben. Also, das hat zweimal gewechselt, zwei verschiedene Personen. Beide Male extrem schlecht. Also, die haben wirklich ein Zehntel von dem gewusst, was ich gewusst hab'. Ich hab' den Leuten echt viel mehr Info geben können. Und das kann's eigentlich nicht sein. ... die nächsten Anlaufstellen wären vermutlich in Linz und die Leute kommen nicht vom Dorf nach Linz. Die Infos müssen vor Ort sein." (EXP MR6)

„R: Meine einzige Erwartung ist, dass den Flüchtlingen am Anfang mehr Information zur Verfügung gestellt wird, in Bezug auf die Gesetze und Regeln, Frauen-, und Männerrechte, Demokratie in diesem Land." (RESP A_m_21_MT 1_1)

" $R$ : Und weiters würde ich empfehlen, wie ich schon gesagt habe, dass man die Flüchtlinge bei der Hand nimmt. Man soll den Frauen helfen und man soll Ihnen sagen, welche Rechte sie haben. Man soll Ihnen sagen, was passiert, wenn sie sich von ihrem Mann scheiden lassen wollen. Wenn sie Probleme haben, wo sie hingehen können. Man soll sie über ihre Rechte 
aufklären. Wenn sie geschlagen werden, dass sie wissen, wohin sie gehen können. Wenn sie belästigt werden, dass sie diese Möglichkeiten haben. Nicht, dass man einfach alle Türen schließt und den Flüchtlingen ... Weil wir kommen ja frisch hierher und können auch nicht die sprache." (RESP A_w_32_ZW_1)

„R: Und sie sollten eine Einrichtung schaffen, wo sie den Menschen, die zum Beispiel aus dem Land kommen oder - keine Ahnung - aus Syrien oder so, wo man diese Menschen über ihre Rechte und Pflichten aufklären sollte. Man sollte Klassen schaffen, wo man über die Gesetze lernt. Damit sie es lernen, dass sie hier diese und jene Dinge nicht machen dürfen. Ich glaube, dass das sehr gut wäre. Sie sollten diesen sagen, dass diese Sachen nicht gehen." (RESP A_w_22_ZW_10))

„R: Diese Menschen sollten ihren Weg wieder weitergehen können und nicht wieder von null anfangen müssen. Und dass die Menschen ein Verständnis dafür aufbringen sollten, wie ich schon gesagt habe, dass die Flüchtlinge aus einer anderen Kultur, einem anderen Sprachraum kommen. Dass sogar die Gesetzeslage in unseren Ländern eine andere ist. Sie sollten wissen, dass die Gesellschaft, die Art der Gesellschaft anders ist. Manche Menschen wissen nicht wie eine bestimmte Arbeiten ausgeführt werden sollte oder wie sie nicht ausgeführt werden sollte. Sie haben diese Arbeit in ihrem Land anders gemacht. Das führt eben zu Problemen. Deshalb ist viel Aufklärungsarbeit nötig. Sie sollten Klassen schaffen, wo sie über bestimmte Themen sprechen. Vor allem für Frauen. Sie sollten es schaffen, dass die Frauen nicht wie in unseren Heimatländern zu Hause bleiben müssen. Dass sie nur Kinder kriegen müssen und solche Sachen. Oder dass sie zumindest den 
jungen Menschen helfen, sich der Gesellschaft anzupassen. Weil wenn die Eltern es nicht schaffen sich zu ändern, die Jungen können das. Diesen muss geholfen werden." (RESP A_w_19_ZW_2)

„R: Man könnte ja auch Audios, also kleine Filme im Internet zeigen, die meisten haben ja jetzt Smartphones, wo das in den Herkunftssprachen wäre, zum Beispiel. ... $J a$, vielleicht auch so mit so einem kleinen Video. Wo man dort natürlich nicht alles auf Persisch geschrieben hat. Oder in der eigenen Sprache, in vielen verschiedenen Sprachen geschrieben hat, sondern [dass es] gesprochen wird." (RESP A_w_29_GR_1)

\section{B) SYSTEMINTEGRATION}

\section{WOHNINTEGRATION}

Einig waren sich Expert/inn/en und Geflüchtete darüber, dass die Wohnintegration eine beträchtliche Herausforderung darstellt, wobei sich Wohnraumknappheit, Mietzinsanstiege in allen urbanen Agglomerationen, Vorurteile vieler Vermieter/innen sowie finanzielle Hürden zu einer Gemengelage akkumulieren, die es Geflüchteten in europäischen Städten erschwert, auf dem Wohnsektor Fuß zu fassen (vgl. Aigner 2016, 2018; EUROCITIES 2016). Die Wohnsituation repräsentiert aber eine wichtige Determinante nicht nur der System-, sondern auch der Sozialintegration und beeinflusst auch die Umsetzung von Maßnahmen in anderen Integrationsbereichen. Die frühzeitige Möglichkeit, in reguläre Wohnsituationen integriert $\mathrm{zu}$ werden, besitzt 
positive Auswirkungen hinsichtlich der sozialen Integration, des Erwerbs von Sprachkenntnissen und einer raschen Integration in den Arbeitsmarkt. In Österreich ist auf dem Wohnungsmarkt der Spielraum für integrationspolitische Eingriffe auf nationaler Ebene sehr begrenzt. Der Bund besitzt keinen Wohnungsbestand, der spezifisch für Geflüchtete zur Verfügung gestellt werden könnte, größere Gemeinden verfügen über einen Stock an Kommunalwohnungen, wobei der Zugang zu diesen in der Regel an bestimmte Aufenthaltsdauern bzw. die Wohnsitzmeldung in der Gemeinde gebunden ist. Es wären aber grundsätzlich mehr Initiativen seitens der Gebietskörperschaften wünschenswert, um Geflüchteten leichter Zugang zu Wohnraum zu verschaffen (vgl. Frey 2011).

Handlungs- und maßnahmenbezogene Empfehlungen

- Bezüglich der Unterbringung in Asylquartieren haben viele Befragte von negativen Erfahrungen berichtet. Basierend auf Kritik sowohl seitens der Expert/inn/en als auch der Geflüchteten ist kleineren Quartieren der Vorzug zu geben und ist zu gewährleisten, dass strikte Qualitätsstandards eingehalten und regelmäßig überprüft werden.

- Im Zusammenhang mit einer dezentralisierten Unterbringung von Geflüchteten ist die Berücksichtigung der Erreichbarkeit von Sprachkursen, Bildungsinstitutionen, Gesundheitsversorgung und der Anbindung an den öffentlichen Verkehr vonnöten. Es ist möglichst frühzeitig eine Wohnsituation anzustreben, die auch einer langfristigen Aufenthaltsperspektive Rechnung 
trägt. Die Expert/inn/en plädieren als integrationsfördernde Maßnahme und zwecks Förderung des individuellen „well-beings“ vor allem für die Möglichkeit der Schaffung „eines eigenen Zuhauses". Bei der Unterbringung sollen die Bleibeperspektive, Familienkonstellationen sowie ethnische und religiöse Faktoren Berücksichtigung finden.

- Das Finden von leistbarem Wohnraum wurde durchwegs als eine sehr schwierige Herausforderung beschrieben. Dennoch wurden von den Geflüchteten nur wenige praxisrelevante Empfehlungen verbalisiert. Vor allem seitens der Expert/inn/en wurde die Forderung nach der Entwicklung von innovativen und umsetzungsorientierten Modellen zur Förderung von bezahlbarem Wohnraum geäußert.

- Der hohe Finanzierungsaufwand für eine Wohnung in städtischen Ballungsgebieten wird als die größte Hürde klassifiziert. Diese kann Weiterbildung und Qualifizierung erschweren und sogar verhindern, obwohl sich daraus für die Geflüchteten sowie aus volkswirtschaftlicher Perspektive langfristige integrationsbezogene Nachteile auf dem Arbeitsmarkt ergeben. Gegensteuernde Maßnahmen, wie finanzielle Unterstützungsleistungen für die Wohnintegration, wären zur Verhinderung zu empfehlen.

- Da dies zum Erhebungszeitpunkt nicht der Fall war, kam und kommt vor allem den NGOs bei der Unterstützung wohnungssuchender Geflüchteter eine wichtige Rolle zu. Einerseits wird empfohlen, durch finanzielle Subventionen den Spielraum der NGOs zu sichern, andererseits wären langfristige, 
nachhaltige, ineinanderübergreifende Maßnahmenpakete von Bund, Ländern und Gemeinden sinnvoll. Für den Zugang zum kommunalen Wohnbau kann eine Verkürzung der vorgeschriebenen Hauptwohnsitzmeldedauer am jeweiligen Ort eine Erleichterung des Zugangs für Geflüchtete bringen.

- Nachhaltige Integrationsmaßnahmen vor Ort können dem Trend zur Binnenmigration in die urbanen Zentren entgegensteuern._Durch ein gezieltes Quartiersmanagement kann etwa die Einbindung in eine funktionierende Nachbarschaft und somit die Sozialintegration gefördert werden. Die Implementierung eines gemeindeübergreifenden Wohnraummanagements durch die Länder könnte die Gemeinden entlasten und zur Koordination und besseren Abstimmung von Angebot und Nachfrage auf dem Wohnungsmarkt beitragen.

\section{Zitate aus den Interviews:}

" $R$ : Unsere Systeme sind einfach nicht darauf ausgerichtet. Dann Benachteiligungen am Wohnungsmarkt. Also Wohnen ist eine große Hürde am Anfang. ...." (EXP MR1)

„R: Also es ist auf jeden Fall wichtig, deswegen haben wir auch diese Initiative, dass man einfach Kontakt hat zur Mehrheitsgesellschaft, dass man nicht in so Massen-WGs untergebracht ist, wo man keinen Kontakt hat zu den Österreichern. Und dass man 
auch ein Zuhause hat und ein bisschen Ruhe hat, sein eigenes Leben so zu gestalten und nicht nur auf das Überleben aus ist. Das sind halt viele Leute, die in diesen Massen-WGs sind, die kommen einfach nicht dazu." (EXP MR3)

„R: ... ich glaub' bei der Wohnungssuche sind für alle sehr, sehr viele Hürden da. Die müssen jetzt nicht auf Papier festgeschrieben sein. Man merkt einfach ständig, dass man nicht die gleichen Chancen hat und dieselben Möglichkeiten bekommt. Das sind, glaub' ich, die größten Hürden, dieser Alltagsrassismus oder diese Vorurteile, denen man überall begegnet .... Es ist schwierig, eine Wohnung am freien Markt zu finden oder andere Nachbetreuungseinrichtungen. Da hat sich auch viel getan in den letzten Jahren, aber trotzdem ist es schwierig einen guten Platz zu finden. Eine Wohnung am freien Markt zu finden ist für unser Klientel eigentlich überhaupt keine Option. (EXP MR2)

„R: Ja, natürlich wäre es gut, wenn es einfach Wohnraum gibt, der zur Verfügung steht geförderter Wohnraum. Auch dass wir hier mehr finanziert werden. Das wäre natürlich auch schön. Dieses Wohnungsproblem war ja schon vorher eine Sache, dass einfach Wohnraum immer teurer wird und es einen Mangel an Wohnraum gibt. Es gibt halt immer wieder so Initiativen oder auch diese "Urbanize Messe", da sind auch immer diese Vernetzungstreffen einmal im Jahr. Und das ist schon immer recht spannend, wie versucht wird, Wohnraum zu schaffen. Aber das ist dann halt auch immer so wenig, dass der Bedarf immer viel höher ist als da entsteht. Also, wenn sich jetzt irgendwer 
überlegt, ein Zinshaus zu kaufen und irgendetwas Cooles zu machen, ist die Warteliste an Leuten, die einziehen wollen, eh schon so lang, dass man da eigentlich nichts machen kann." (EXP MR3)

"R: Sie wissen im Vorfeld nicht, was passiert. Sie sind nicht informiert. Wissen zwar, dass sie [UMF] ausziehen müssen, wenn sie 18 sind, aber sie werden darauf nicht vorbereitet. Sie wissen nicht wohin, das kann überall sein." (EXP JJ)

„R:... Erfahrungen okay, ... negative gibt's auch, ..., weil momentan als ich von der Grundversorgung dann wegziehen musste, finanzielle Probleme hatte ich schon, damit ja, weil Wohnungen Kaution Provision, ... damit habe ich Schwierigkeiten gehabt, ..." (RESP GS8)

„I: Und du bist dann vom Burgenland gleich nach Wien gekommen?

$R$ : Ja, dann hab' ich den Status bekommen und bin nach Wien. Ich habe eine Wohnung gefunden. Die Caritas hat mir geholfen mit Kaution und Provision. Ich habe ja für die Caritas in der Brunnenpassage am Anfang freiwillig gearbeitet. Ich hatte ja kein Geld, aber habe dann die Wohnung bekommen, im 21. Bezirk ..." (RESP MR 1)

„R: Der Sprachkurs war ein Problem, und dass wir eine Wohnung finden. Meine Frau war schwanger und hat zwei Mal ein Kind verloren, wie wir noch im Heim gelebt haben. Das Leben im Heim war sehr schwierig. Es waren sehr viele Ratten dort. Wir waren 13 Monate lang im Heim. Es waren insgesamt 50 Menschen, die in den Containern gewohnt haben. 
Dann haben wir diese Garage gefunden. Die Hauseigentümerin hat uns sehr viel geholfen, um diese Garage in eine Wohnung umzugestalten. Wir haben jede Woche, Samstag und Sonntag daran gearbeitet. Meine Frau und ich haben beide dran gearbeitet." (RESP MRMT3)

\section{ARBEITSMARKTINTEGRATION}

\subsection{BERUFLICHE INTEGRATION}

Die berufliche Integration repräsentiert, neben der Integration durch Bildung und dem Erwerb der Landessprache, womit enge Zusammenhänge bestehen, das zentrale Handlungsfeld im Kontext der Systemintegration. Die Integration in den Arbeitsmarkt impliziert aber auch vielfältige Herausforderungen, da Geflüchtete oft nicht die Anforderungen des österreichischen Arbeitsmarktes erfüllen und vor Aufnahme einer Erwerbstätigkeit einen längeren Trainingsprozess absolvieren müssen. Die Wichtigkeit adäquater integrationspolitischer, auf den Arbeitsmarkt fokussierter Maßnahmen ist vor allem angesichts zahlreicher Benachteiligungen, mit denen sich Geflüchtete konfrontiert sehen (vgl. Fetz 2011), hervorzuheben.

Kann eine Integration in den Arbeitsmarkt rasch erfolgen, so erleichtert diese den Aufbau sozialer Netzwerke auf dem Arbeitsplatz und als „learning by doing" die Internalisierung westlicher Wertsysteme (Cheung und Phillimore 2013). Folglich kann das Spektrum an Fördermaßnahmen im Feld der beruflichen Integration nicht breit genug sein. Die prioritäre Wichtigkeit von Optionen für die möglichst frühe 
Aufnahme einer Erwerbstätigkeit wurde in den Interviews durchgehend hervorgehoben.

Bei den Afghan/inn/en verteilen sich bei den in den vergangenen Jahren Angekommenen, zum Unterschied von den während der 1980er- und 1990er-Jahren Geflüchteten, die zum Großteil höhere Bildungsabschlüsse besaßen, die Bildungsqualifikationen bimodal ${ }^{2}$ (vgl. Buber-Ennser et al. 2016; Wiedner et al. 2018). In Anbetracht des heterogenen Qualifikationsniveaus (vgl. AMS 12-012016), des Faktums, dass die Afghan/inn/en das niedrigste Bildungsniveau mitbringen (50\% kein oder nur Grundschulabschluss) sowie des häufig mangelnden "Systemwissens" über Österreich und dessen institutionelle Strukturen, ist davon auszugehen, dass deren Erwerbsintegration mehr Zeit als bei anderen Herkunftsgruppen in Anspruch nehmen wird.

Die Berufsqualifikation im Herkunftsland (bei einem hohen Prozentsatz nicht Afghanistan, sondern der Iran oder Pakistan) wurde bei den meisten durch „on-thejob-training“ erworben. Seit 2016 eingeleitete Bestrebungen zur Ergänzung informell erworbener Qualifikationen durch Angebote formaler Zusatzausbildung in Österreich zeigen eine raschere Verwertbarkeit derselben auf dem Arbeitsmarkt (Hosner et al. 2017). Neben mangelnden Qualifikationen ist besonders die Aufenthaltsunsicherheit (viele Afghan/inn/en erhalten

\footnotetext{
${ }^{2}$ Einem relativ hohen Anteil von Personen ohne jegliche formale Bildung steht ein ebenfalls beträchtlicher Anteil höher gebildeter Personen gegenüber.
} 
nur subsidiären Schutz) ein Grund dafür, dass Unternehmen geflüchtete Afghan/inn/en nur zögerlich einstellen. Dabei wären in kleineren und mittelständischen Unternehmen vielfach sogar geeignetere Möglichkeiten der beruflichen Integration gegeben als in Großbetrieben. Der Arbeitgeber/innenperspektive wurde jedoch in wissenschaftlichen Analysen bislang eher wenig Beachtung geschenkt (vgl. WKO 21-03-2017). Die während der Projektlaufzeit nach und nach eingeführten Integrationsmaßnahmen haben die Arbeitsmarktchancen von Geflüchteten erheblich erhöht (arbeitplus 02-10-2017; Kurier 15-03-2018).

Der Mismatch zwischen Qualifikationen aus dem Herkunftsland (oder den in Pakistan/Iran erworbenen) und den faktischen Erwerbsmöglichkeiten in Österreich ist ausgeprägt. Die LODA-Befragung bestätigt, dass asylberechtigte oder subsidiär schutzberechtigte Afghan/inn/en weitgehend im niedrigqualifizierten Sektor beruflich tätig sind. Dies gilt oft auch bei guten, im Heimatland erworbenen Qualifikationen. Allerdings betonen die Befragten durchgehend, dass sie unbedingt und vor allem rasch arbeiten wollen und sie darüber hinaus durchaus bereit sind, auch dequalifizierende Tätigkeiten auszuüben.

Positiv zu vermerken ist, dass sich nach der „refugee crisis" ein breit angelegtes Engagement durch Bündelung von Maßnahmen unterschiedlicher Träger (AMS, WK, AK, WIFI, Schulen und Schulverwaltung) sowie durch Kooperationen mit engagierten Unternehmer/inne/n, den Sozialpartner/inne/n und Ehrenamtlichen herausgebildet hat. Auch unsere Daten bestätigen internationale Forschungsbefunde (z.B. Ryan 2011; Fóti und Fromm 2016), dass nämlich soziale 
Netzwerke für Geflüchtete oft entscheidend bei der Suche nach einer Beschäftigung sind. Einige Befunde deuten darauf hin, dass durch „social ties" mit Inländer/in/nen gefundene Jobs den über Anzeigen oder Vermittlung des AMS gefundenen sogar überlegen sind, was Entlohnung und Qualitätsparameter angeht (Cheung und Phillimore 2013). Allerdings besteht das Risiko, dass bei der Jobsuche nur über ethnische Netzwerke der Communities primär Positionen am unteren Ende der Beschäftigungshierarchie vermittelt werden.

Mit dem Ansteigen der Zahlen an Geflüchteten wurden immer öfter Vorschläge in Richtung einer forcierten Unterstützung von Start-ups verbalisiert. Dies wurde als ein Ausweg aus der oft schwierigen Anerkennung von Qualifikationen für Angestelltenpositionen propagiert und sollte der heimischen Wirtschaft neue Ideen und Stimuli bringen. Allerdings hat sich laut AMS-Vorstand Kopf die Vermutung, dass die Geflüchteten eine hohe Neigung zur Selbständigkeit mitbrächten, nicht bestätigt. Das AMS hat ein Gründerprogramm aufgelegt, 2016 lag bei diesem die Zahl der Absolvent/inn/en bei 22 Personen, mit Ende 2018 werden es rund 50 sein. Das von der Stadt Wien geförderte Start-up-Programm wurde bis 2018 von 230 Interessierten in Anspruch genommen. ${ }^{3}$

3 Regina Bruckner: 40 Prozent der Wiener Unternehmer haben Migrationshintergrund - Der STANDARD, 13. Oktober 2018, S. 28. [derstandard.at/2000089238859/40-Prozent-der-Wiener-

Unternehmer-haben-Migrationshintergrund]. 
Handlungs- und maßnahmenbezogene Empfehlungen zur Förderung der Arbeitsmarktintegration

- Durchwegs wurde von Expert/inn/en und Geflüchteten die Erleichterung eines raschen Zugangs zum Arbeitsmarkt auch für Asylwerber/innen, vor allem aber für Asylberechtigte und subsidiär Schutzberechtigte, als erstrebenswertestes Ziel im Kontext der auf den Arbeitsmarkt bezogenen Maßnahmen angesprochen. Die Arbeitsmarktintegration sollte idealerweise auch den Kriterien der Nachhaltigkeit und Zielgerichtetheit entsprechen sowie optimal an die mitgebrachten Voraussetzungen und Rahmenbedingungen angepasst sein. Hier wurde von Expert/inn/en auch eine realitätsbezogene Neudefinition der Kategorie „Arbeitsfähigkeit“ angeregt.

- Der raschere Arbeitsmarktzugang steht auch in einem engen Zusammenhang mit der Empfehlung nach einer Beschleunigung der Asylverfahren. Eine wichtige Voraussetzung bildet die Anerkennung von mitgebrachten Qualifikationen, bei der seitens der Expert/inn/en ebenfalls die Beschleunigung und Vereinfachung der Verfahren empfohlen wird.

- Geflüchteten ist nach Möglichkeit eine fundierte Berufsausbildung zu ermöglichen. Dies zeitigt längerfristig für die Betroffenen wie für die Wirtschaft positivere Effekte als eine rasche Integration in ungelernte Hilfstätigkeiten mit geringen Weiterbildungs- und Aufstiegschancen. Als wichtiges Desiderat sollten Ausbildungschancen auch für Ältere geboten werden. 
- In den Interviews kam vor allem die hohe Motivation der Geflüchteten zur Aufnahme einer Erwerbstätigkeit zum Ausdruck. Diese sollte nach Möglichkeit sinnvoll genutzt werden und in diesem Zusammenhang kann durch passende Maßnahmen auch die erforderliche (aber nicht immer im Vordergrund stehende) persönliche Bereitschaft zur (Weiter-) Qualifizierung gefördert werden.

- Die Arbeitssuche ist mit zahlreichen Hürden und erheblichen Frustrationen verbunden. Arbeitslosigkeit stellt eine häufige Erfahrung vieler Respondent/inn/en dar. Die Wichtigkeit der Unterstützung seitens NGOs und engagierter Freiwilliger wird seitens der Expert/inn/en betont. Gerade im Sinne der beruflichen Integration ist ein möglichst differenzierter Förderkatalog für berufsspezifische Aus- und Weiterbildungsmaßnahmen zu empfehlen.

- Aus den Interviews geht hervor, dass viele Geflüchtete die Bildungs- und beruflichen Möglichkeiten in Österreich erheblich überschätzen. Der durch formale Qualifikationsnachweise stark reglementierte Arbeitsmarkt und das damit eng verzahnte Bildungssystem sind für viele (vor allem geringer gebildete) Befragte nur schwer nachzuvollziehen. Die Information über die real offenstehenden Möglichkeiten auf dem Arbeitsmarkt (aber auch in anderen Bereichen der Systemintegration) sollte daher so systematisch wie möglich und zu einem möglichst frühen Zeitpunkt des Aufenthaltes erfolgen. Dies erleichtert den Betroffenen die zeitgerechte Adaption von zu optimistischen individuellen Vorstellungen 
hinsichtlich des Erwerbs höherer Bildung und beruflicher Perspektiven.

- Gerade auch für Personen mit beträchtlichen Qualifizierungsmängeln (v.a. in der afghanischen Herkunftsgruppe) sollten Möglichkeiten zum frühen Kennenlernen beruflicher Praxis in Österreich organisiert werden, auch wenn noch keine rasche Integration in "echte“ Beschäftigungsverhältnisse möglich ist.

- Drop-out-Risiken und Ausbildungszeiten könnten durch eine ausgeprägtere modulare Aufgliederung der Berufsausbildung und Möglichkeiten, mehrere Ausbildungsmodule zu kombinieren, positiv beeinflusst werden. Dazu zählt auch eine möglichst breite Palette an raschen und langfristigen Möglichkeiten zur Inklusion von Geflüchteten in Unternehmen.

- In Bezug auf den Kompetenzcheck stehen vorwiegend positive Beurteilungen seitens der Expert/inn/en kritischen Meinungen einiger Geflüchteter gegenüber. In diesem Feld hat sich seit der Befragung aber vieles verändert, sodass unsere Daten nicht mehr den aktuellen Status quo widerspiegeln können. Hierzu eine Empfehlung abzugeben, würde daher weitere Recherchen zum Status quo erfordern, welche aus zeitlichen und organisatorischen Gründen nicht realisierbar sind.

- Zu den sinnvollen Maßnahmen zählt des Weiteren die Erleichterung von Unternehmensgründungen. Start-ups und die Migrant Entrepreneurship sollten also weiterhin umfassend gefördert werden. Überzogene Erwartungen hinsichtlich der quantitativen Dimensionen der damit einhergehenden Beschäftigungseffekte sind laut den 
Expert/inn/en aber nicht gerechtfertigt. Die qualifikatorischen, legistischen und finanziellen Hürden auf dem Weg zur selbstständigen Erwerbstätigkeit sind im Vergleich zu den Herkunftsgesellschaften in Österreich sehr hoch.

- Die Arbeitsmarktchancen im urbanen bzw. ländlichen Raum sind abhängig von der mitgebrachten Qualifikation sowie den angestrebten Tätigkeiten. Ein Trend zur Binnenmigration in die Städte, v.a. nach Wien, nach Erhalt des Asylbescheids lässt sich aus den Interviews klar herauslesen. Dieser ist nicht selten die Folge häufig unrealistischer Hoffnungen hinsichtlich eines leichteren Wohnungs- und Arbeitsmarktzugangs. Eine realitätsnahe Einschätzung ihrer faktischen Arbeitsmarktchancen ist den Interviewten in Unkenntnis der Rahmenbedingungen aber vielfach nicht möglich. Allerdings wurden in einigen der Expert/inn/eninterviews, aber nicht seitens der Respondent/inn/en Politikempfehlungen im Sinne einer Gegensteuerung der Land-StadtBinnenmigration abgegeben. Räumliche Dekonzentration kann als Mittel zur Förderung der regionalen Arbeitsmarktintegration eingesetzt werden. Mit fundierten Informationen über die faktischen Chancen und Möglichkeiten kann der Binnenmigration nach Wien wahrscheinlich am effizientesten entgegengesteuert werden.

- Eine (zeitlich befristete) Residenzpflicht, wie etwa in einigen skandinavischen Staaten (ehemals bzw. bis jetzt) gebräuchlich, wird unterschiedlich bewertet. Positive Auswirkungen in Richtung einer Reduktion der Binnenmigration wären von fixen 
„Andockpunkten“ für Geflüchtete auch außerhalb Wiens zu erwarten. Vor dem Hintergrund der Expertise des ISR sowie der medialen und politischen Diskussion ist zu sagen: Obwohl die Gründe für diese stadtzentrierte Migration individuell stark divergieren, wären hier zusätzliche Maßnahmen der Gegensteuerung wichtig und werden auch seitens einiger Expert/inn/en befürwortet. Diese können sich etwa in Form einer Ausweitung der systematischen Informationsarbeit des AMS, der WKO und der NGOs zu den faktischen Chancen auf den regionalen Arbeitsmärkten manifestieren. Vor allem in Bezug auf Mangelberufe und Lehrstellen in solchen wird hier bereits seit längerem Informationsarbeit betrieben, auch zusätzliche Anreize (z.B. finanziell oder wohnintegrationsbezogen) für die Annahme von offenen Stellen in ländlichen Räumen wären sinnvoll. Die Wirtschaftskammern und das AMS sowie engagierte Unternehmer/innen haben in punkto regionaler Abstimmung von Arbeitskräftenachfrage und -angebot bereits viel erreicht - dieser erfolgreiche Weg sollte unbedingt fortgesetzt werden. Last but not least, muss die Arbeitsmarktintegration in Kleingemeinden auf dem Land Hand in Hand mit systematischen Maßnahmen der sozialen Integration gehen, um die Geflüchteten nachhaltig in die lokale Gemeinschaft einzubinden. Hier kommt den engagierten Ehrenamtlichen und lokalen Vereinen eine eminente Vermittlungs- und Betreuungsrolle zu.

- Trotz der im Vergleich zu den beiden anderen Herkunftsgruppen höheren Anteile an Personen mit geringen beruflichen Qualifikationen verläuft die 
Arbeitsmarktintegration der Afghan/inn/en nicht zwingend schlecht. Sie sind sogar eher als andere Gruppen von Geflüchteten bereit, in Hilfsberufen tätig zu werden und können auf diese Weise in der Gastronomie bzw. im Fremdenverkehr (besonders in den westlichen Bundesländern) sogar leichter als manche Hochqualifizierte Anstellungen finden.

- In Bezug auf die Arbeitsmarktintegration ist nach den Grundsätzen der Nachhaltigkeit und Langfristigkeit zu handeln. Durch krasse Dequalifizierung im Rahmen rascher Erwerbsintegration gehen aus längerfristiger Perspektive wertvolle qualifikatorische Potentiale für die österreichische Wirtschaft verloren. Segmentation und ethnische Segregation ist dem Arbeitsmarkt inhärent und kann realistischerweise nicht gänzlich vermieden werden. Dennoch sollte entgegengesteuert werden.

\section{Zitate aus den Interviews:}

„R: Möglichst rasch zum Arbeitsmarkt zugelassen zu werden und eine leistbare Wohnung finden. Der Rest findet sich dann schon, spätestens über den Arbeitsplatz. Ich habe auch bei der gemeinnützigen Beschäftigung gemerkt, dass Integration super über den Arbeitsplatz funktioniert. Auch dort wird Kultur vermittelt, weil sie einfach von den Kolleg/inn/en vorgelebt wird. Syrische und irakische Männer waren nie ein Problem bei der gemeinnützigen Beschäftigung im Umgang mit Frauen, anders ist es im Privatbereich .... EXP: Mein Wunsch an die Bundesregierung: Deutschkurse und andere Maßnahmen ab dem 1. Tag, nicht erst ab Asylrecht. 
Zugang zum Arbeitsmarkt sofort, die Leute wollen arbeiten, sie sind fleißig, geben wir ihnen doch rechtlich die Möglichkeit." (EXP RS)

„R: Diese Ungewissheit haben wir gemerkt, die nagt an den Flüchtlingen. Dieses Nichtwissen, wie wird über meinen Fall entschieden, darf ich hierbleiben, muss ich zurück, macht es überhaupt Sinn noch weiter einen Deutschkurs zu besuchen, weil ich vielleicht eh abgeschoben werde, ist doch eh alles sinnlos, warum lassen sie mich nicht arbeiten, in meiner Heimat war ich auch fleißig und selbständig, konnte für mich selber sorgen. Warum darf ich hier nicht arbeiten? Die Menschen fühlen sich gekränkt, auch in ihrer Würde, weil sie sagen: ,Ich bin ein anständiger Mensch immer gewesen, ich möchte auch hier anständig sein und etwas arbeiten für mein Geld. Ich will nicht alles geschenkt bekommen von Euch, bitte lasst mich arbeiten.' Es ist also sehr traurig, wenn ich den Leuten sagen muss: ,Ihr dürft aber nicht arbeiten, das ist leider nicht erlaubt in Österreich.' Wenn ich mir etwas wünschen dürfte, dann eine ganz rasche Änderung der Rechtslage, das wäre sehr wichtig. Jetzt versuche ich halt, rechtliche Schlupflöcher auszunutzen, die mir der Gesetzgeber freundlicherweise gelassen hat, wie z.B. über gemeinnützige Hilfstätigkeiten .... Also einfache Tätigkeiten, das können auch Menschen machen, die kein hohes Bildungsniveau haben". (EXP RS)

„R: Also, es wäre wie gesagt super, wenn man gleich regional etwas besser zuordnet, gleich nach der Einreise. Dann so rasch wie möglich mit Deutschkursen beginnt. Auch während des Asylverfahrens. Da ist jetzt eh einiges schon verbessert worden. Dann idealerweise, wenn es nicht gelingt, das Asylverfahren innerhalb von sechs 
Monaten abzuschließen, nach sechs Monaten einen Arbeitsmarktzugang mit Beschäftigungsbewilligung ermöglicht. Für die jugendlichen Asylwerber mit hoher Anerkennungswahrscheinlichkeit generell die Lehre in allen Berufen zu ermöglichen. Ich glaub', es braucht eine transparente Arbeitsmarktinformation: Wo gibt es die Jobchancen? [Man braucht eine ] gute Berufsinformation. Sehr rasche gute Kompetenzerfassung. Und da muss man halt die Zielgruppen anschauen. Wenn es z.B. um das Thema Pflichtschulabschluss geht, also quasi man muss halt wirklich nach Zielgruppen schauen. Was bringt jemand mit? Und wie kann ich diese Person so rasch wie möglich in Richtung österreichischen Arbeitsmarkt, österreichische Gesellschaft bringen? Der zweite Bereich ist aus meiner Sicht das Thema Asylverfahren, Arbeitsmarktzugang für Asy/werber. ... Was jetzt die Jugendlichen betrifft. Also die Möglichkeiten, eine Lehre absolvieren zu können. Das ist ein wichtiger Punkt." (EXP MR7)

„R: Also so: ,Okay, jetzt habe ich einen super Hauptschulabschluss gemacht, jetzt geht es an die Lehrstellensuche. Ich habe nur diesen Status, Das heißt, ich darf nur aus diesen Berufen auswählen und hab nicht den freien Zugang.' ... ,Ich bin auf der Lehrstellensuche und weiß, mit meinem Nachnamen wird es vermutlich auch schwieriger.' Oder Bewerbungen sind wahrscheinlich auch deprimierender. Man kriegt wahrscheinlich noch mehr Absagen, wie jemand, der hier geboren ist. ... Arbeitsmarkt, da haben sie gar keine Chance. Es gibt Mangelberufe, wo es dann heißt, ohne Hauptschulabschluss würde es auch gehen, aber irgendwie hat es bis jetzt keiner geschafft." (EXP JJ) 
„R: Arbeitsmarktintegration ist [wichtig] für ein selbstbestimmtes Leben, das heißt ja auch unabhängig von Sozialleistungen, von Transferleistungen. Okay, da kann man drüber streiten, aber ja natürlich bringt es auch einen Grad an Selbstständigkeit mit. ... Und da müsste man sich anschauen, wo sind diese Lücken und wo braucht es da Regelsysteme und wo müssen Regelsysteme adaptiert werden?" (EXP MR1)

„I: Welche Aspekte sind für Integration wichtig? R: Zugang zu Deutschkursen. Also dass man Deutsch lernen kann. Arbeitsmöglichkeiten RR: Genau! Ich find'auch, es gibt halt sehr viele administrative, bürokratische Schranken und wenn du einfach den Zugang hast zu einer weiteren Ausbildung oder zum Arbeitsmarkt. Also viele Leute treten auf der Stelle ... weil sie nicht arbeiten können, weil sie nicht studieren können, und dann ist es einfach schwierig." (EXP MR3)

„R: Wir beobachten, dass es für die Unternehmen mittlerweile, wenn man es zugespitzt formuliert, eigentlich aus ihrer Sicht eine Loose-loose-Situation geworden ist. Wenn eine große Handelskette 50 Asylwerbern Lehrstellen gibt, dann sind leider Gottes die Zeiten vorbei, wo man da auf Facebook viele positive Rückmeldungen bekommt. Das hat sich schon stark geändert. Die Unternehmen, die mit uns zusammenarbeiten, sind da vorsichtiger geworden. Ich kann jetzt nicht mehr so leicht über die Social Responsibility da jetzt für mich als Unternehmer einen Mehrwert lukrieren. Am Anfang sind viele Unternehmen gekommen und wollten unterstützen, das ist auch weiterhin so, aber es ist nicht mehr so leicht für größere Unternehmen, dafür in der Öffentlichkeit einen Bonus zu kriegen. Das hat sich schon ein bisschen geändert. Ich 
weiß es selbst, wenn da irgendwas online gegangen ist, wenn man die Postings liest, [die] sind jetzt nicht alle die besten. Das ist jetzt nicht mehr so. Der erste Punkt ist, dass es für die Unternehmen - natürlich jetzt rein wirtschaftlich gesehen - ein Mehraufwand ist. Da ist jemand, mit dem muss man wahrscheinlich noch Deutsch lernen, da wünscht man sich auch eine Unterstützungsstruktur. Dass da vielleicht auch jemand ist, der vermittelt. Es ist sicherlich ein bissl mehr Arbeit, und es ist gleichzeitig nicht mehr so, dass es in allen Fällen publik gemacht wird. Das macht es für die Unternehmen nicht einfacher. ... Der Abschluss einer gelungenen Arbeitsmarktintegration ist, wenn die Menschen auch wo arbeiten." (EXP KT)

„R: Dass es schneller geht mit der Anerkennung von Berufsausbildungen. Ich weiß nicht, wie viele syrische Ärzte hier noch rumsitzen und nicht arbeiten dürfen. Also das wär'schon wichtig und die Angebote und die Kurse. Auch die Ausbildungsmöglichkeiten auch für Ältere zu öffnen. Ich glaub', man muss sich grad um die Jungen kümmern. Die mit 50, 60 sind, glaub' ich, schwierig zu integrieren. Die Jungen könnte man noch einsetzen und ausbilden in den Mangelberufen, die es in Österreich gibt und da könnte man schon ein bisschen schneller sein." (EXP MR8)

„R: Es müssten auch zahlreiche bürokratische Hürden abgebaut werden, z.B. eine Beschleunigung der Nostrifizierungsverfahren und generell in der Berufsanerkennung. Den Geflüchteten diesbezüglich das Leben leichter machen [und] würde die Integration fördern. Natürlich muss die Qualität der Arbeit erhalten bleiben. Wir haben Leute im Land, die sind jetzt da. Tun 
wir etwas für sie und mit ihnen. Geben wir ihnen die Chance, etwas Sinnvolles zu machen." (EXP RS)

"R: Wie haben bei den Personen vom Bildungsniveau her eine extreme Spreizung, da gibt es sehr viel mehr mit einer Matura, es gibt aber auch sehr viel mehr mit maximal Pflichtschulabschluss. Weil eben dieses Zwischensegment einfach fehlt, was bei uns diese duale Ausbildung ist. Da arbeiten wir schon viel daran, Aufklärungsarbeit zu machen: Was ist denn das? Warum ist das sinnvoll? Dann auch noch das ganze Drumherum. Es gibt natürlich aus diesen Ländern sehr viel mehr Personen, die sich gern selbständig machen würden. Und das machen wir aber von Anfang an, in den Kompetenzchecks; in dem einen Kurs kommt alle zwei Monate eine Steuerberaterin und erklärt einmal sozusagen was es heißt, wenn ich mich in Österreich selbständig mache. Dann haben wir noch ein nachgelagertes System, das Step-to-Business, auch in den Muttersprachen, wo dann noch einmal geschaut wird, kann man da überhaupt einen Businessplan machen, ja oder nein? Wenn das positiv ist, dann gehen sie in das Unternehmensgründungsprogramm vom AMS. Da bleiben nicht viele übrig. Das Ergebnis ist aber für beide Seiten positiv, weil die, die es dann schaffen, werden es wahrscheinlich auch schaffen, und die, die es nicht schaffen, denen wurde dann auch wirklich dreimal gesagt, das ist einfach unrealistisch und dann kann man das vielleicht auch irgendwie abhaken und zum nächsten Punkt übergehen. In den Kompetenzchecks machen wir auch sehr viele Workshops: österreichischer Arbeitsmarkt; Wie stelle ich mich vor?." (EXP KT)

„R: ... unser Zugang, dass man sehr, sehr rasch bestehende Kompetenzen und Berufserfahrungen schon 
nach der Einreise nach Österreich erheben sollte und das dann eben auch bei der geographischen Zuteilung berücksichtigt. Wenn jemand z.B. Erfahrung hat im Tourismus, in der Gastronomie, Hotellerie im Herkunftsland, dann wär' es doch sinnvoll, diese Person eher in Richtung Salzburg und Tirol zu transferieren und versuchen sie dort zu halten. Und wenn jemand [Erfahrung hat] im technischen Bereich, dann in Oberösterreich. Also, dass man darauf auch Rücksicht nimmt und dann sukzessive auch die Asylwerber auch schon auf eine Tätigkeit in der Region vorbereitet. Vor allem dann, wenn sie eine hohe Anerkennungswahrscheinlichkeit haben. Das wäre extrem wichtig. Und dann kann man natürlich auch über Dinge wie Residenzpflicht etc. nachdenken. Einfach um das besser auszugleichen, weil ich glaub' das führt in keine gute Richtung - diese unterschiedlichen Konzentrationen." (EXP MR7)

„R: Und ich glaub", da wär ${ }^{\prime}$ es in zweierlei Hinsicht wichtig, ein bisschen ein transparenteres Bild zu geben, welche Möglichkeiten man hat. Natürlich sind es nicht nur die Jobchancen, die eine Sogwirkung von Wien aus in Richtung der Bundesländer ausstrahlen. Aber es geht auch um Angebote von Deutschkursen und vielleicht gewisse Unterstützungsleistungen, die es in der Vergangenheit noch nicht so gegeben hat, die jetzt aber sukzessive aufgebaut werden. Und ich würd' mir wünschen und hoffen, dass das dazu beiträgt, dass nicht so viele Asylberechtigte und subsidiär Schutzberechtigte dann nach Wien gehen, weil die Jobchancen sind nicht unbedingt gegeben. Also die sind viel mehr gegeben, das wissen wir, weil wir arbeitsmarktpolitisch den Überblick haben, dass es viel besser wäre in Oberösterreich, Tirol, 
Salzburg zu bleiben, weil da gibt es wirklich Jobmöglichkeiten." (EXP MR7)

„R: $(m):$... dass wir arbeiten dürfen. Wenn die Menschen nicht arbeiten dürfen und keinen Sprachkurs haben, dann werden sie sich anders beschäftigen. Entweder werden sie schlechte Taten begehen oder andere Dinge. Sie werden depressiv und gewöhnen sich an die staatliche Unterstützung. Wenn man keine Beschäftigung hat und keine Arbeitsstelle, ist es sehr schwierig. Ich kenne Leute, die nur schlafen. R: (w): Ich habe die gleiche Meinung. Sie sollen uns erlauben, dass wir hier wachsen und weiterkommen, uns weiterentwickeln. Die Menschen, die kommen, sind sehr talentiert und können vieles bewegen. Aber anstatt, dass wir uns hier weiterentwickeln können, entwickeln wir uns zurück. Die sollen mit uns nicht wie im Iran umgehen. Wir würden sehr gerne ganz normal leben hier. Wir flüchten, weil wir es mussten und nicht, weil wir es wollten." (A_m+w_26_27_MRMT3)

\subsection{BESCHLEUNIGUNG UND STEIGERUNG DER TRANSPARENZ DER ASYLVERFAHREN}

Die lange Dauer der Asylverfahren wirkt sich auf viele Bereiche der System- und Sozialintegration negativ aus. Abgesehen von der psychischen Belastung besteht gemäß der Einschätzung der befragten Expert/inn/en Einhelligkeit darüber, dass die lange Dauer der Asylverfahren besonders der raschen Arbeitsintegration sowie der Weiterbildung und längerfristigen Höherqualifizierung abträglich ist. 
Allerdings haben die seit dem „Rückstau“ von 2016/17 behördlicherseits gesetzten Bemühungen in Richtung einer Abkürzung der Asylverfahren und somit der Reduktion der Wartezeiten auf einen Job in der Befragung bereits sehr positiv Erwähnung gefunden. Lange Verfahrenszeiten betreffen nach Aussage der Befragten nach wie vor allem die Afghan/inn/en, von denen viele ohnehin nur subsidiären Schutz zuerkannt erhalten. Dies gilt weitgehend auch für die Iraker/innen. Die syrische Herkunftsgruppe ist davon viel weniger betroffen.

Handlungs- und maßnahmenbezogene Empfehlungen zur Förderung der Wohnintegration

- Durch eine weitere Beschleunigung der Asylverfahren ist ein zusätzlicher Positiveffekt auf die berufliche und soziale Integration zu erwarten.

- Ein aus der Ungleichbehandlung der Herkunftsgruppen resultierendes "Zweiklassensystem" im Asylverfahren ist hinsichtlich seiner Auswirkungen auf die Integrationsverläufe der davon Betroffenen als ungünstig zu bewerten.

- Unsere Daten belegen, dass besonders „kritische Passagen" in der Integrationsbiographie Geflüchteter existieren. Dies gilt in erster Linie für den herausfordernden Übergang zwischen dem Status des/r Asylwerber/s/in und des/r Asylberechtigten. Für die bessere Bewältigung dieser spezifischen Phase werden eine intensivere, gezieltere Beratung und Begleitung empfohlen. Sollte mit den Potentialen der 
existierenden Beratungsstellen nicht das Auslangen gefunden werden, so wäre deren Aufstockung anzuraten.

- Die mit der Dauer der Verfahren und der Unsicherheit des Ausgangs verbundenen psychischen Belastungen sowie das Nichtvorhandensein eines selbst erarbeiteten Einkommens bedingen neben einer Ressourcenvergeudung auch psychische Erkrankungen und können bei einigen Betroffenen sogar zu erhöhter Devianzneigung führen. Hier wird angeraten, gezielt mittels Maßnahmen gegenzusteuern.

\section{Zitate aus den Interviews:}

"R: Natürlich ist es schwierig für die Asylsuchenden. Die Asylverfahren dauern sehr, sehr, sehr lange, im Durchschnitt mittlerweile glaube ich so zwischen zwei und drei Jahre wartet man auf einen Bescheid in der ersten Instanz. Das ist eine sehr lange Zeit, und in der Zeit darf man nicht arbeiten, man hat keinen Zugang zum Arbeitsmarkt." (EXP RS)

„R: Ich glaub", dass unsere Angebote viel zu langsam anrollen, dass es zu wenig Sprachkurse gibt. Diese ganzen Behördengänge bis jemand Asyl bekommt, das dauert alles so lange. Da ist es schon unglaublich, wie langsam das funktioniert. Und wie lange diese Leute herumsitzen und nichts tun dürfen und halb durchdrehen dabei. Mein Mann ist in der Perchtoldsdorfer Flüchtlingshilfe sehr engagiert. Der sagt: ,Das ist unglaublich. Die werden wahnsinnig. Die sitzen da, seit 
dieser großen Welle seit zwei Jahre. Und haben keinen Bescheid, dürfen nicht arbeiten, dürfen kein Geld verdienen. Furchtbar, ja!"“ (EXP MR8)

„R: ... mit der jetzigen politischen Situation ist das ein bisschen schwierig, dass man da zu irgendetwas motiviert. ... weil man einfach das Gefühl hat, dass diese Thematik nur mit Problemen in Verbindung gebracht wird. Auch in den Medien einfach sehr wenig Positives berichtet wird, und einfach geschaut wird, dass nicht noch mehr Leute kommen, auf einer europäischen Ebene ... Weil jetzt auch einfach mit den vielen Abschiebungen auch Leute abgeschoben werden, die schon ewig lange in Österreich sind, oder wir Leute kennen, die seit Jahren im Asylverfahren sind, schon super Deutsch können und immer noch nicht wissen, ob sie hierbleiben können. Ich glaube, das ist psychisch auch sehr schwer, wenn du nicht weißt was passiert. Da versuchst du dich $z u$ integrieren, indem du Deutsch lernst und dich ehrenamtlich engagierst und Kontakte knüpfst usw. und dann wirst du abgeschoben. Also das ist einfach, grad wenn die Regierung ja fordert, dass man sich integriert und dann macht man das und dann wird man abgeschoben, das ist einfach kontraproduktiv." (EXP MR3) 


\subsection{ARBEITSMARKTSERVICE}

Die Bewertungen des Kursangebotes sowie der Interaktionen mit dem AMS fallen äußerst kontroversiell aus. Sowohl Expert/inn/en als auch Geflüchtete kritisieren mehrfach die aus ihrer Perspektive mangelnde Unterstützung durch Berater/innen des AMS, z.B. den Zugang zu weiterführenden Deutschkursen oder anderen Ausbildungen, die mangelnde Kontinuität der Kurse, das geforderte Niveau, welches für Personen mit geringer Schulbildung zu hoch angesetzt sei, Inhalte und didaktische Vermittlungskonzepte usw. Auch die Umgangsformen und Interaktionsmodi, ein Mangel an Empathie oder die Unfreundlichkeit mancher Betreuer/innen wurden seitens der Geflüchteten wiederholt beschrieben. Hohe Arbeitsbelastung und knappe für die einzelnen Klient/inn/en zur Verfügung stehende Zeitressourcen spielen hier unzweifelhaft eine Rolle. Dem stehen allerdings auch sehr positive Bewertungen bezüglich der Betreuer/innen sowie des Niveaus und der Inhalte der angebotenen Kurse gegenüber. In einigen Fällen berichten Geflüchtete, dass sie beobachtet hätten, dass der Umgang mit inländischen KlientInnen „anders" sei. Im Sinne der Ausgewogenheit in den Bewertungen ist festzustellen, dass vor allem im AMS auf Basis der im Rahmen der "refugee crisis" akkumulierten Erfahrungen Lernprozesse eingesetzt haben, die Beratungen wesentlich erweitert und zahlreiche Bemühungen in Richtung einer Verbesserung des Angebotes gestartet wurden (vgl. Gatterbauer 2019).

Handlungs- und maßnahmenbezogene Empfehlungen 
- Ungeachtet einer Reihe von Verbesserungen bezieht sich eine Empfehlung auf das kontinuierliche Monitoring des Beratungsangebots und der Art und Weise der sozialen Interaktionen zwischen Berater/inne/ $\mathrm{n}$ und Klient/inn/en. Ohne stressbedingte Interaktions- und sprachliche Verständigungsprobleme jemals völlig ausschalten zu können, wäre seitens des AMS eine neutralere Kommunikation mit den Klient/inn/en, klarere Begründungen, z.B. bei Nichtgewährung eines Kurses, anzustreben, da damit viele Missverständnisse im Vorhinein vermieden werden könnten.

\section{Zitate aus den Interviews:}

„R: Zum AMS gehen die Jungs erst, wenn sie subsidiären Schutz bekommen haben. Als Asylwerber haben sie mit dem AMS nichts zu tun. Nachdem sie subsidiären Schutz bekommen haben gibt es Maßnahmen wie "Pole Position". Es gibt große Beschwerden über den Ablauf. Es ist auch schwierig, wenn sie in NÖ angemeldet sind, nach Wien zu kommen, was eigentlich ihr Ziel wäre. Das funktioniert aber nicht so einfach." (EXP JJ)

„I: [Welche]Rückmeldungen [gibt es] von den Verantwortlichen in den Kursen des AMS? R: Eigentlich auch nicht andere als in anderen Kursen. Die teilweisen Befürchtungen, dass es dort mehr Probleme gibt aus den unterschiedlichsten Gründen, haben sich so nicht eingestellt. Das ist wie in anderen Kursen auch. Es hängt schon sehr viel zusammen mit den Sprachkenntnissen. 
Sobald wer besser Deutsch spricht, ist alles einfacher, dann kommt es auch nicht so leicht $z u$ Missverständnissen. ... Wir haben muttersprachliche Personen gesucht, die dorthin gehen und Aufklärungsarbeit machen, die auch in jede neue Gruppe hineingehen und in der Muttersprache die generellen Regeln besprechen. Und dann auch in Situationen, in denen es Diskussionsbedarf gegeben hat, vermittelt haben. Das hat mehr Druck rausgenommen und war im Endeffekt sicher auch der bessere Zugang. Das haben wir jetzt auch aufgestockt. Wirklich einfach Personen, die in den Bereichen, wo die Menschen einfach noch zu schlecht Deutsch sprechen und auch vielleicht noch nicht so lange in Österreich sind, so ein Verbindungsglied, die vermitteln können, aufklären können und einfach auch klarer dezidiert sagen können, was man macht und was man nicht macht." (EXP KT)

„R: $A 1, A 2$ und $B 2$ habe ich schon absolviert und derzeit bin ich beim AMS und möchte eine Ausbildung machen, damit ich einen Beruf erlernen kann und dann in dem Bereich arbeiten kann. ... Ja, ich war sehr zufrieden, die Kurse waren ganz gut. ... Positiv war, dass ich die Sprache gelernt habe und Negatives gab es eigentlich nicht, es war alles gut, die Kurse sind sehr gut, aber das einzig Negative ist, dass AMS sich sehr viel Zeit lässt bei der Zuteilung weiterer Kurse. Zirka ein Jahr habe ich einen A2-Kurs gemacht und drei Jahre hatte ich nichts zu tun, dann habe ich wieder einen Kurs bekommen. Wenn man durchgehend einen Deutschkurs besucht, dann lernt man die Sprache auch schnell und ist dann schnell vorbereitet für die Arbeit. ... Trainer waren Österreicher, alle waren sehr nett, haben gut unterrichtet, das 
bestätige ich, dass sie sehr gut unterrichtet haben." (A$\mathrm{m}-38, \mathrm{GS} 5-2$ )

"R: Solche Dinge habe ich oft bei manchen Organen [Anm: Organisationen] gesehen. Zum Beispiel beim AMS. Manche Berater beim AMS, die Deutsch sprechen. Ich habe zum Glück ein paar Berater gesehen, die Persisch sprechen beim AMS, die sehr nett sind. Bei den deutschsprachigen Beratern habe ich ... ein wenig das Gefühl, ... dass sie denken, dass man nichts weiß. Sie haben irgendwie keinen Respekt oder vieles ist ihnen egal. Aber ich bin mir sicher, dass wenn ein Deutschsprachiger mit ihnen spricht, dass sie sich sicher anders verhalten würden." (A_w_19_ZW_2)

„R: Es wäre schöner, wenn das AMS und die Berater ein bisschen mitfühlender und empathischer wären. Oft war es so, dass wenn mein Vater einen Termin beim AMS hatte, und er kann nicht gut Deutsch, dass sein Berater auch deutschsprachig war. Mein Vater konnte sich nicht viel verständigen und sein Gegenüber auch nicht. Sie geben Ihnen einfach Kurse, mehr überprüfen sie nicht. Meine Mutter hat noch schlechtere Deutschkenntnisse als mein Vater. Sie wollten meine Mutter in einen Kurs für B1 hinschicken. Ich bin dann mit ihr hingegangen und man hat sie abgeprüft und sie hat die Prüfung nicht bestanden. Sie machen manchmal so komische Sachen, die ich nicht verstehe. Wieso prüfen sie nicht wirklich nach, was diese Person kann und was nicht? Ich finde, dass man genauer nachschauen und nachprüfen sollte, was ein Mensch für Qualitäten hat, was er kann." (A_w_19_ZW_2)

„R: Ja, ich habe viele Deutschkurse vom AMS besucht. ... $J a$, ich war zufrieden, da ich regelmäßig einen 
Deutschkurs vom AMS bekam und habe viele Deutschkurse besucht, jedoch die Sprache, da ich viel älter gewesen bin, konnte ich nicht viel sprechen. Die Deutschkurse waren temporär, das war ein Fehler von AMS. Sie hätten uns langandauernde Deutschkurse geben müssen. Sie schickten uns zu einem Deutschkurs für vier Monate, danach waren wir neun Monate arbeitslos, dann haben wir wieder einen Deutschkurs bekommen und alles, was wir in der Vergangenheit gelernt haben, haben wir wieder vergessen. Das ist der Fehler gewesen, wenn wir einen Kurs nach dem anderen hintereinander bekommen hätten, hätten wir die Sprache gelernt, so war das aber nicht ..." (A_m_66_GS3)

„R: Ich erwarte oder wünsche mir, dass man eventuell eine Organisation schafft, die hilft Ausbildungen zu finden oder bei der Arbeitssuche hilft. Das AMS ist eine Art Kontrollorgan, das nur prüft, ob man eine Arbeit gefunden hat oder nicht. ... Ja, bis jetzt war es so. Und man sollte Berater einstellen, die helfen sollen, dass man seine Ausbildungspläne realisieren kann. Ich hatte beispielsweise vor kurzem einen Termin beim AMS und das AMS wusste Bescheid, dass ich mir Mühe gegeben hatte einen Job zu finden. Ja, ich habe zwar keine Ausbildung oder keine Arbeit gefunden, aber ich war nicht untätig. Ich habe gesucht. Ich habe nicht den ganzen Tag geschlafen oder nur Freizeitaktivitäten gehabt. Ich habe meinen Tag in Kursen verbracht und damit eine Ausbildung oder eine Arbeit zu finden. Mein Berater hat mich aber versucht zu überreden, zum McDonalds zu gehen und dort als Putzfrau zu arbeiten. Welchen Beitrag soll ich denn leisten, wenn ich als Putzfrau beim McDonalds arbeite? ... Ich habe ihm dann gesagt, dass ich nicht putzen möchte. Er soll mir bitte 
helfen eine Ausbildung zu finden. Wenn er mir eine Ausbildung finden würde, würde ich am nächsten Tag sofort hingehen. I: Wie war für Sie dann der Umgang des Beraters mit Ihnen? B: Sehr schlecht." (A_W_21_ZW_61)

\section{BILDUNGSSYSTEM}

Der Zugang zu Bildung ist eine fundamentale Voraussetzung für die Integration in das Erwerbsleben und den sozioökonomischen Aufstieg. Der Bildungssektor schafft wie die Arbeitsintegration die Grundlagen für eine nachhaltige Systemintegration sowie damit in einem Konnex stehend die Integration in die Aufnahmegesellschaft (vgl. Titelbach et al. 2013). Das Erlernen der jeweiligen Landessprache repräsentiert eine wesentliche Voraussetzung für die Teilhabe am gesellschaftlichen Leben (vgl. Chiswick und Miller 2015; Dustmann und Glitz 2011). Da die unterschiedlichen Dimensionen der Integration in einer Wechselwirkung zueinander stehen, kann ohne Beherrschung der Landessprache kaum eine erfolgreiche Platzierung auf dem Arbeitsmarkt und ein sozialer Aufstieg erfolgen (Alam 2008: 72).

Mit dem Ende der starken Flüchtlingsimmigration der Jahre 2015/16 hat sich der Fokus der öffentlichen Diskussion von der Unterbringung und Versorgung hin zur dauerhaften Integration verlagert. Während in der Frühphase des Aufenthaltes vor allem der rasche Erwerb von Grundkenntnissen der deutschen Sprache im Fokus stand, haben mit der Zeit längerfristige Bildungsangebote und berufliche Qualifikationen als wichtige Maßnahmen zur Integration an Gewicht 
gewonnen (Wößmann 2016). Hervorzuheben ist, dass die interviewten Geflüchteten durchwegs eine hohe Bildungsbereitschaft mitbringen, sehr bestrebt sind Deutsch zu lernen und auf dem Arbeitsmarkt Fuß zu fassen (vgl. auch Buber-Ennser et al. 2016; Kohlbacher et al. 2017).

Eine Voraussetzung für die Bildungsintegration stellt die Anerkennung im Ausland erworbener Qualifikationen und Kompetenzen dar (Biffl 2012). Die komplexen Probleme, wie die Einhaltung von Fristen, zeitaufwendige bürokratische Procederes, finanzielle Aufwendungen, die Beschaffung in Verlust geratener Zeugnisse etc., wurden in den LODA-Interviews häufig erwähnt.

Im Bereich der Bildung wurde seit dem Projektstart einiges bewegt. Bildungseinrichtungen auf allen Ebenen und im gesamten Bundesgebiet haben zahlreiche neue Angebote geschaffen. Auch die Anerkennung der Bildungsabschlüsse aus den Herkunftsländern konnte beschleunigt werden.

Bezogen auf den Bildungssektor waren es in unserer Erhebung primär die Deutschkurse, die im Rahmen politischer Empfehlungen seitens der Expert/inn/en, aber auch der Geflüchteten angesprochen wurden. Der Altersstruktur der Respondent/inn/en sowie den diversen Backgrounds der Expert/inn/en entsprechend konnten nur sehr wenige Best-Practice-Vorschläge zu Bedarfen und Herausforderungen im Regelschulwesen gesammelt werden.

Handlungs- und maßnahmenbezogene Empfehlungen zur Förderung der Bildungsintegration 
- Während der langen Asylverfahren besteht auch kein offizieller Zugang zum Bildungssystem. Dies führt in vielen Fällen dazu, dass die Zeit förmlich „davonläuft" und die Möglichkeit des Nachholens von Bildungsabschlüssen schon allein bedingt durch den Zeitfaktor immer unwahrscheinlicher wird. Im Interesse der Arbeitsmarkt- und Bildungsintegration ist eine Verfahrensbeschleunigung anzustreben.

- Ein bereits in der ersten Projektfokusgruppendiskussion und in etlichen Expert/inn/eninterviews angesprochener Bedarf manifestiert sich in der raschen Anerkennung/Nostrifikation von Qualifikationen aus dem Herkunftsland sowie der Vereinfachung des Nachholens von Bildungsabschlüssen.

- Anhand des vorliegenden empirischen Materials sehen wir eine noch stärkere Vernetzung der verschiedenen relevanten Organisationen und Akteur/inn/e/n im schulischen und beruflichen Ausbildungsbereich als ein erstrebenswertes Ziel im Interesse der Geflüchteten an.

- Ein Mangel an relevanten Informationen wurde sowohl von den Expert/inn/en als auch den Geflüchteten wiederholt verbalisiert. Daraus resultiert die Empfehlung eines breiten und detaillierten Informationsangebots über die Strukturen und Möglichkeiten des Bildungssystems sowie personenbezogene, individualisierte Unterstützung und Beratung bei der Wahl geeigneter und realisierbarer Ausbildungs- und Berufspfade.

- Anknüpfend an die Interviews wird die Entwicklung eines adäquaten Instrumentariums angeraten, um das Erlernen der deutschen Sprache stärker mit 
praxisorientierten, berufsrelevanten Elementen anzureichern bzw. beide Sphären (Spracherwerb sowie berufliche Umsetzung) zielorientiert miteinander zu verknüpfen. Augenmerk ist des Weiteren auf die Kontinuierlichkeit des Angebots, aber auch des Besuchs von Deutschkursen durch die Geflüchteten zu legen.

- Die Ausweitung des Wiener Modells („Deutsch ab dem ersten Tag") auf alle Bundesländer kann gestützt auf Statements der Expert/inn/en ebenfalls empfohlen werden. Der Zeitraum des Asylverfahrens sollte also bereits sinnvoll zum Deutschlernen genutzt werden. Allerdings stellt sich basierend auf Erfahrungen aus der Praxis angesichts der Unsicherheit des Verfahrensausgangs auch das Problem der kontinuierlichen Aufrechterhaltung der Lernmotivation. Dieses harrt einer Lösung.

- Die Berufstätigkeit von Frauen wird durchwegs befürwortet und meist ökonomisch begründet, vor allem höher gebildete Respondentinnen sehen darin auch einen Schritt in Richtung ihres Empowerments und gesellschaftlicher Teilhabe. Allerdings werden Frauen schon beim Deutschlernen mit dem Problem konfrontiert, dass zu wenige Deutschkurse in Kombination mit Kinderbetreuungsmöglichkeiten existieren. Frauenspezifische Bildungsangebote in Kombination mit Kinderbetreuungsmöglichkeiten sind also vonnöten. Ihr Spektrum hat sich im Vergleich zu früheren Fluchtwellen (z.B. jener aus Bosnien-Herzegowina) zwar erheblich erweitert, dennoch besteht primär außerhalb der urbanen Räume nach wie vor ein gravierender Mangel. Kindergärten schließen in kleineren Gemeinden oft bereits um 13:00 Uhr. 
- Basierend auf Kritik seitens der Geflüchteten ist eine noch gezieltere Diversifizierung des Deutschkursangebotes nach Aufnahme- und Leistungsfähigkeit, Bildungshintergrund und Altersgruppen, Fremdsprachenkenntnissen im Interesse einer Optimierung des Outputs der Sprachkurse zu empfehlen.

- Im Zusammenhang mit den Deutschkursen ist auch eine Systematisierung externer Inhalts- und Qualitätsevaluierungen anzuraten. Dem Erhebungszeitraum mit seinen hohen Zahlen an Geflüchteten entsprechend wurden längerfristige und quantitativ ausreichende Angebote an Deutschkursen auch in ländlichen Räumen sowie alternative Lernangebote (z.B. E-learning-Angebote) gefordert. Diese Empfehlung müsste aktuell angesichts stark gesunkener Bedarfszahlen noch speziellen räumlichen Evaluierungen unterzogen werden.

- In Übereinstimmung mit wissenschaftlichen Erkenntnissen und Expert/inn/enstatements sollte Mehrsprachigkeit expliziter auch als Gewinn und Zusatzqualifikation angesehen werden, denn in Österreich wird zu einseitig auf den Erwerb der deutschen Sprache fokussiert. Es mangelt an einer positiven Bewertung von Mehrsprachigkeit.

- Wir sehen auch die Notwendigkeit professionellen psychologischen Supports bei bildungsbezogenen (Motivations)problemen, als Hilfe zum Durchhalten auf dem Bildungsweg, zum Kompensieren von Enttäuschungen bei erfolglosen Bewerbungen oder wenn sich angestrebte Berufslaufbahnen als nicht realisierbar herausstellen. 


\section{Zitate aus den Interviews:}

"R: Das ist eine große Hürde. Warum ist es bei unseren Burschen so, die wirklich viel investieren und einen großen Teil in ihre Bildung stecken, um hier weiterzukommen und dann stehen sie einfach immer wieder vor Hürden. Also so: ,Okay, jetzt habe ich einen super Hauptschulabschluss gemacht, jetzt geht es an die Lehrstellensuche. Ich habe nur diesen Status, das heißt, ich darf nur aus diesen Berufen auswählen und hab' nicht den freien Zugang.'" (EXP MR2)

„R: ..... das Deutschlernen war für die Leute wirklich immer so eine wahnsinnige Hürde. $\mathrm{Zu}$ einem Deutschkurs zu kommen und das, also finde ich, ist in Wien also wirklich, wirklich toll. Deutsch von Anfang an, dass die Leute auch eine Fahrkarte bekommen, also zumindest am Anfang, wenn sie Deutschkurse gemacht haben. Also das hat es wirklich wahnsinnig erleichtert, weil sich die Leute dadurch automatisch ein bisschen willkommener geheißen, äh, gefühlt haben. Also das war wirklich, finde ich, also das war so eine sicher teure Maßnahme, aber das war irgendwie so eine Maßnahme, die sehr gut das Klima irgendwie [zum Positiven verändert hat] (...) ,Jetzt müssen wir schon warten, also jetzt kann man zumindest die Zeit nutzen', das war, das war wichtig, finde ich." (EXP MR 10)

„R: Deutschkurse sind also einmal ganz wichtig für die Integration ab dem ersten Tag. ... Eben Integration $a b$ dem ersten Tag mit Fokus auf Arbeitsmarktvorbereitung ..." (EXP RS) 
„R: Oder es ist auch schon schwierig, Deutschkurse mit Kinderbetreuung anzubieten. Das ist wirklich eine Herausforderung, wenn ich sage: Ich habe Mütter, die jetzt vielleicht auch nachkommen, weil der Mann war die Vorhut und hat das Asylrecht bekommen, jetzt kommt die Frau mit den drei Kindern nach. Dass wir diese Frau dann in einen Deutschkurs schicken. Weil der Mann hat im günstigsten Fall schon Arbeit gefunden, ist berufstätig und kann sich daher nicht um die Kinder kümmern, und wir wollen, dass die Frau einen Deutschkurs besucht, dann muss ich aber ihre Kinderbetreuung sicherstellen. Und dann brauche ich, wenn die Kinder schon größer sind, am Nachmittag einen Hortplatz. Das sind alles Logistikprobleme: Hortplätze, Kinderbetreuungsplätze sind eine große Herausforderung. (EXP RS)

„R: ... weil offiziell ist dann alles super aber praktisch funktioniert es noch immer nicht. Sowas, also ja. Also die Kommunikation immer zu Lasten der Asy/werber/innen. Ich denke mal, es gibt Länder, wo diese ... Mehrsprachigkeit irgendwie auch mehr verankert ist ... (EXP MR10)

„R: Dann Deutschkurse. Die sind am Anfang über freiwillige Helfer gelaufen. Das war aber ein höchst kompliziertes Thema. Ich hab' mich da dann irgendwann ausgeklinkt. Mein Bruder hat das gemacht. Freiwillige Helfer haben das angeboten und das dann zu koordinieren war schwierig, weil es waren auch ganz viele Analphabeten dabei. Dann war eine dabei, die fließend Englisch konnte. Mit der war es überhaupt leicht, weil die hat schon mal eine andere Sprache gelernt. Es war einfach voll das Chaos. Das ist einfach ähnlich wie bei der Volkshilfe. Da braucht es 
Organisation vom Staat. Das kann man nicht organisieren." (EXP BL)

„R: Beim Sprachkurs würde ich sagen, die Art und Weise, wie der Lehrer unterrichtet hat. Und auch die Art und Weise, wie er mit uns umgegangen ist. Er hat nämlich auf die Menschen, die sich schwer getan haben beim Lernen. Klar, er hat diese Personen gekannt, mit diesen hat er schon zwei Kurse gemacht, aber wenn diese einmal nicht vorbereitet waren, dann hat er immer wieder erwähnt, ob diese Personen wissen, wie viel Geld für sie schon ausgegeben wurde für die Kurse. Seine Art war sehr frech. Und andere, die besser waren, die hat er dann sehr gut behandelt. Zu mir war er sehr nett. Aber andere, die sogar älter waren als der Lehrer selbst, die hat er auch respektlos behandelt. Diese hat er sehr schlecht behandelt. Diese Personen konnten es einfach nicht. Ich verstehe nicht einmal, wieso sie einen B2-Kurs gemacht haben. Sie konnten nicht einmal viele A2Sachen. Aber sie waren im B2-Kurs. Und das ist meiner Meinung nach etwas ganz Schwieriges. Dann habe ich eines Tages gefragt, ob die Personen nach einer abgelegten Prüfung den B2-Kurs machen dürfen oder wie das genau war. Und es war einer im Kurs, den ich kannte. Dieser hatte den B1-Test zweimal abgelegt, aber nicht geschafft und waren trotzdem im B2-Kurs. Und deswegen hat er nichts gelernt. Wenn er B1 nicht konnte, dann konnte er auch beim B2-Kurs nicht viel lernen. Ich finde dieses System nicht gut. Aber der Kompetenzcheck ... Das, was ich vom Kompetenzcheck nicht erwartet habe, war, ... Weil ich dachte, dass die Klassen nach dem Alter aufgeteilt sind und ältere Damen zusammen in einem Kurs sitzen, während jüngere eine andere Klasse besuchen. Aber dann habe ich gesehen, dass ich 18 bin 
und im Kurs aber 50-Jährige, 60-Jährige gesessen sind und solche Personen. Und dass es zwei Klassen gleichzeitig gab. Aus diesem Grund konnte ich auch insgesamt dann 4 Stunden beim Kompetenzcheck nicht teilnehmen, weil ich gleichzeitig meinen Deutschkurs hatte. Und die Stundenpläne waren sehr schlecht." (A_w_19_ZW_2)

„R: Über die Methoden der Deutschkurse sind wir zufrieden gewesen, aber nicht so sehr, die Fehler der Deutschkurse bestanden darin, dass die Deutschkurse [mit Unterbrechung] stattfanden. Man hat uns kurze Zeiten mit Deutschkursen beschäftigt, dann hatten wir lange Zeiten Pausen, obwohl wir weitermachen wollten. Das war eine Fehlfunktion des Systems, obwohl sie viel investiert haben, sind diese Investitionen ziellos und fehlerhaft gewesen. Hätten wir zwei oder drei Jahre durchgehend Sprachkurse wie in einer Schule gehabt, dann wären wir sicherlich weitergekommen. Denn wir wären dann all die Zeit durchgehend in Kontakt mit Menschen und dem Lernstoff gewesen. Dies wäre sicherlich ein sehr positiver Anhaltspunkt. Anstatt Deutschkurse mit Unterbrechungen zu geben, wäre es viel besser gewesen, gezielte langanhaltende Deutschkurszeiten zu haben. Dies war ein großer Fehler des Systems. Ich habe ein Ansuchen, nämlich dass man genauso diese Fehler künftig bei Flüchtlingen nicht mehr macht." (A_w_52_GS_9)

„R: Also in Österreich einzuleben ist das Wichtigste. Dass man als Flüchtling in Österreich was beiträgt, damit man sich auch zufrieden ist mit sich selbst und dadurch auch in einem sozialen Austausch kommen kann und dementsprechend auch die Sprachkenntnisse verbessern kann. Die Sprache spielt für mich eine sehr, sehr wichtige 
Rolle, aber ich kann mich auch, ich kann mir auch vorstellen, dass nicht jeder Flüchtling imstande ist, die Sprache so schnell als möglich zu erwerben, weil es ist individuell und von Situation zu Situation, die sie erlebt haben anders. [Durch die Erfahrungen in der] Vergangenheit, ist deren Habitus [geprägt], was sie dann in weiteren Entwicklungsjahren, in einer neuen Stadt hindert,... also es ist sehr wichtig, dass sie die Sprache an erster Stelle lernen, an der zweiten Stelle, an dem sie arbeiten müssen, ist die Arbeit". (A_w_26_GS 2)

"R: Ich erwarte mir von ihnen, dass sie uns helfen eine Arbeit zu finden damit ... Den A2-Kurs haben wir. Und wenn man dann den B2-Kurs machen sollte, dann sollten sie ... bei dieser Prüfung wird wenig Zeit gegeben. Es gab viele Hindernisse und unser Gehirn funktioniert nicht so wie das eines Jugendlichen. Man kann nicht schnell etwas lernen ... und schnell und perfekt etwas lernen. Entweder man gibt einem bei der Prüfung mehr Zeit oder es sollte auf die kleineren Sachen nicht viel Wert gelegt werden. Diese Dinge sind sehr schwer. Ich habe die A2Prüfung abgelegt und alles war so klein geschrieben. Und ich werde älter und meine Augen sind nicht mehr so gut. Ich habe nur schwarze Pünktchen gesehen. Und dass sie zum Beispiel bei den Prüfungen nicht so streng sein sollen und dass sie mehr Zeit geben sollen und dass sie nicht viel Wert auf Kleinigkeiten legen. Aber Gott sei Dank habe ich meine Prüfung geschafft. Ich kann es immer noch nicht glauben, weil die Zeit war sehr knapp ... in kurzer Zeit musste man zwei Seiten schnell ausfüllen und schnell erledigen. Innerhalb einer halben Stunde ... und wenn wir nicht fertig wurden, dann haben sie einfach die Blätter abgeräumt. Die jungen Menschen, die Englisch können und zur Schule gegangen sind, die 
können das. Aber wir, die nicht zur Schule gegangen sind, wir können das nicht. Weil wir danach ... Sie sollten nicht Jung und Alt gleich prüfen. Es ist sehr schwierig. Wenn Sie die Vorschriften ein bisschen ändern könnten und anstatt einer halben Stunde eine Stunde prüfen und keinen Wert auf Kleinigkeiten legen, dann wäre es viel einfacher. Ansonsten ist es sehr schwierig. Ich habe den $B 1$ nicht gemacht, weil er sehr schwer ist. Schwer für einen und man leidet darunter. Wie soll man das schaffen, wie kann man das lernen? Man kann nachts nicht einschlafen, weil man sich dauernd fragt, wie man die Prüfung ablegen soll und wie man das durchstehen soll. Ich bin zum Beispiel 52 Jahre alt, mein Sohn ist auch krank und ich habe hundert andere Probleme." (A_w_51_ZW_4)

\section{GESUNDHEITSWESEN}

Hinsichtlich der Zahl und der Breite der Palette an Handlungsempfehlungen rangierte das Gesundheitswesen weit hinter den anderen Feldern der Systemintegration. Hinsichtlich der Qualität der medizinischen Versorgung in Österreich wurde so gut wie keine Kritik verbalisiert. In erster Linie in Bezug auf das Gesundheitswesen genannt, aber auch die anderen relevanten Systeme in Österreich betreffend, wurde die Installierung von Maßnahmen zur Information und Explikation der Funktionsmechanismen und Strukturen, der Zugangsvoraussetzungen und der Interaktionen zwischen Repräsentant/inn/en dieser Systeme und den Bürger/inne/n als sehr wichtig erwähnt. Dieses institutionsbezogene Wissen erleichtert den Geflüchteten wesentlich den Umgang mit 
Krankenhäusern, Ambulanzen, den Mitarbeiter/inne/n der Krankenkassen, Ärzt/inne/n, Therapeut/inn/en etc. Erwartungshaltungen können auf diese Weise auch beeinflusst und überzogene in realistische Bahnen gelenkt werden.

Die Sprachbarriere spielt im Gesundheitswesen eine die generelle Kommunikation, Diagnostik und Therapie ganz besonders beeinflussende und vielfach einschränkende Rolle, damit hängt auch eine gewisse Ungeduld mancher Ärzt/inn/en bei zeitaufwendigen Gesprächen mit der Notwendigkeit von Übersetzungshilfe zusammen. Darüber hinaus wurden in den Interviews kaum Beschwerden über essentielle Mängel geäußert; am ehesten noch über den erlebten Zeitmangel bei niedergelassenen Ärzt/inn/en. Durchwegs positiv wurde sowohl von Expert/inn/en aus den NGOs als auch von den Geflüchteten die prompte und effiziente Behandlung der Patient/inn/en im österreichischen Gesundheitswesen hervorgehoben. Analog zu den in den vorangegangenen Abschnitten bereits behandelten Systemen wird Informationsmangel als grundlegendes Negativum konstatiert.

Handlungs- und maßnahmenbezogene Empfehlungen zur Förderung der Integration im Sektor des Gesundheitswesens

- Zur Kompensation des bestehenden Informationsdefizits werden muttersprachliche Infokampagnen über Struktur und Funktionsweisen des österreichischen Gesundheitssystems empfohlen.

- Der dringlichste Bedarf im Gesundheitswesen besteht an muttersprachlichen Übersetzer/inne/n 
und Dolmetscher/inne/n. Dies betrifft das Arzt/Ärztin-Patient/inn/en-Gespräch, den stationären Krankenhausaufenthalt, genauso aber das Verstehen schriftlich ergehender Befunde und das Lesen der Beipackzettel von Medikamenten. Von Expert/inn/en angeraten wird die intensivere Nutzung von bereits seit 2013 (damals noch im Pilotversuch) installierten digitalen Möglichkeiten der Involvierung von Video-Dolmetscher/inne $/ \mathrm{n}^{4}$.

- Im engen Konnex zu Maßnahmenempfehlungen im Bildungssystem steht die schnellere Nostrifikation von im Ausland abgeschlossenen Medizinstudien. Gerade in Syrien entspricht das Niveau der Ausbildung hohen Standards und syrische Ärzt/inn/e/n könnten zum Beispiel den Mangel an Allgemeinmediziner/inne/ $\mathrm{n}$ in vielen ländlichen Räumen Österreichs kompensieren helfen.

- Hinsichtlich der psychologischen Betreuung wäre ein höheres Maß an „trauma awareness“ zu empfehlen. Dieser Aspekt wäre generell in allen Integrationsbereichen stärker zu berücksichtigen. Personen, die Traumata zu verarbeiten haben, agieren selbst in Alltagssituationen mitunter anders als Nichttraumatisierte. So viel als möglich an professioneller Unterstützung ist hier unsere dringende Empfehlung.

Zitate aus den Interviews:

${ }^{4}$ Vgl. Käfer (22-08-2016). 
„R: Da gibt es viele Herausforderungen. Erstens einmal kommen die Leute aus anderen Gesundheitssystemen. Die kennen unseren Zugang nicht. Das ist der Grund, warum im AKH in den Ambulanzen Migranten in großer Zahl sitzen. Weil sie unser System nicht kennen. Unser System des niedergelassenen Arztes ist in anderen Ländern nicht gang und gäbe. ... Die zweite große Herausforderung ist sicher das Kommunikationsproblem. Einerseits sprachlich, aber auch von der Bedeutung. Es gibt so Dinge, die kann man zwar sprachlich klären und trotzdem versteht man sich nicht." (EXP MR8)

„R: Es war aber ganz wichtig, dass am Anfang immer jemand von uns dabei war. Also von den freiwilligen Helfern. Also ich oder der Rest von meiner Familie und die anderen freiwilligen Helfer. Sonst wär' es ganz schwierig geworden, weil die Verständigung einfach null war, gar keine sprachliche Verständigung da war. Ich hab' es vorher zwei Stunden ausgedeutscht, was das Problem war, bin dann hingegangen und hab' es übersetzt. War dann dabei beim Arztbesuch. Ich hab' dann schon ein bisschen rauslesen können, was die Leute meinen, auch wenn ich die Sprache nicht verstanden habe. Das war ganz wichtig. Also ohne da mitzugehen, artet es im Chaos aus und das ist das, was die Ärzte dann so nervt. Was ich voll verstehe. Die waren natürlich zum Teil auch ein bisschen unfreundlich, weil einfach alles so lang dauert, bis da mal was rauskommt. Das geht natürlich viel schneller mit jemandem, der einfach Deutsch spricht." (EXP MR6)

„R: Also es müssen prinzipiell die Leute immer ihre Dolmetscher mitbringen. Also ganz oft in Krankenhäusern, da versuchen wir jetzt grad sehr viel, 
weil in den Wiener Krankenhäusern gibt es jetzt VideoDolmetsch, und da gibt es ein System und die Ärzte sind meiner Meinung nach nur zu faul, um das anzuwenden. Das gibt es und die Klientinnen werden trotzdem weggeschickt oder es erklärt ihnen die Freundin, die natürlich nicht medizinisch ausgebildet ist." (EXP MR10)

„R: Das ganze Gesundheitswesen funktioniert in Afghanistan anders. Da ist die Selbstversorgung in erster Linie vorhanden und es ist unvorstellbar, dass jemand im Krankenhaus ist und dass er nicht täglich besucht wird. D.h., wenn die Häuser in X. sind und die Kinder in Y. oder sonst wo im Spital sind dann geht die ganze Mannschaft jeden Tag hin, um sie zu besuchen. Sie verstehen einfach nicht, dass da schon jemand vor Ort Essen und Trinken hat, sondern man geht hin, sitzt da und verbringt die Zeit zusammen. Für sie sind das traumatische Erlebnisse. Erstens, weil sie dem Arzt nicht vertrauen was auch daher kommt, dass, wenn man in Afghanistan im Krankenhaus ist, man nicht weiß, ob man da wieder rauskommt und vor allem wie, und was da alles passieren kann. Sie haben extreme Angst vor Operationen. Allein zum Zahnarzt zu gehen ist immer ein Theater." (EXP JJ)

„R: Also, was sinnvoll wäre und was es in den Krankenhäusern ja zum Teil gibt, wäre ein Dolmetscher. Einen kommunaleren Dolmetscher, der entweder dabei ist oder der über Video, Computer, über den Bildschirm auch erreichbar ist." (EXP MR8)

„R: Nein, das gibt es jetzt. Also nicht bei praktischen Ärzten und beim praktischen Arzt, da versteh' ich das schon, aber die Krankenhäuser, das AKH, von dem AKH wissen wir, also da gibt es eine eigene, also er/wir wissen 
das jetzt auch, da gibt es eine eigene Person, die ist dafür zuständig. Nur im AKH wissen das nicht alle, also das ist dann einfach ärgerlich, weil offiziell ist dann alles super, aber praktisch funktioniert es noch immer nicht. ... Also die Kommunikation [ist] immer zu Lasten der Asylwerber/innen. Ich denke mal, es gibt Länder, wo diese (...), in denen die Mehrsprachigkeit irgendwie auch mehr verankert ist." (EXP MR10)

„R: Ok, wenn jemand erst seit zwei Wochen in Österreich ist, wie soll er sich beim Arzt wirklich verständigen und dem Arzt erklären welche Probleme er hat? Natürlich schafft er das nicht. Deswegen wäre es besser, wenn man einen Dolmetscher bereitstellen würde oder ... Es gab wirklich viele Leute, die das gebraucht haben und die monatlich einen Check-up machen mussten. Viele schwangere Frauen mussten monatlich untersucht werden und diese mussten halt alleine zum Arzt gehen, wenn sie einen Dolmetscher gefunden haben. Sie mussten sich immer jemanden finden, der Ihnen helfen konnte. ... Organisationen sollten schauen, dass sie den Menschen in solchen Angelegenheiten helfen und nicht, dass diese Menschen sich selbst Hilfe suchen müssen. Natürlich ist es eine Mehrarbeit, aber man muss diesen Menschen helfen. Ich finde, es sollte geholfen werden bei Sprachproblemen..." (A_w_19_ZW_2)

\section{SOZIALE INTEGRATION}


gesellschaftspolitischer Konsens darüber, dass die längerfristige Abschottung von Geflüchteten von der Mehrheitsgesellschaft und die Etablierung etwaiger Parallelgesellschaften als nachteilig zu bewerten ist. Dies entspricht auch den Intentionen aller befragten Geflüchteten, die sich explizit viele Kontakte zur österreichischen Mehrheitsbevölkerung wünschen. Soziale Netzwerke und Kontakte zur österreichischen Gesellschaft wirken sich in allen Integrationsfeldern unterstützend und fördernd aus, etwa beim Deutschlernen, bei der Wohnungs- und Arbeitssuche, in der Orientierung an möglichen Vorbildern und der Bewältigung von Ausbildungswegen. Für die langfristige Integration in die Gesellschaft leisten Buddy-Systeme im Tandem mit Ehrenamtlichen einen unschätzbaren Beitrag. Zu diesem Zweck sind jegliche Maßnahmen, welche Kontakte mit Angehörigen der Aufnahmegesellschaft forcieren sowie solche, welche generell die Offenheit der lokalen Bevölkerung für eine Kontaktaufnahme fördern, sehr zu begrüßen.

Ein Basiswissen über europäische und lokale Grundwerte und deren historische Entstehungs- sowie Bedingungskontexte wird zwar in den Wertekursen vermittelt, darüber hinaus können Werte und lokale Gepflogenheiten in sozialen Alltagsinteraktionen aber viel eindrücklicher vermittelt werden als selbst durch das beste Informationsmaterial.

Im Feld der sozialen Integration kommt Ehrenamtlichen eine besonders wichtige Rolle zu, denn sie können verbal und durch ihr Verhalten vermitteln, welche gesellschaftlichen Konventionen gelten und welches Sozialverhalten im Regelfall einzuhalten ist und welche Reaktionen erwartet werden. 
Handlungs- und maßnahmenbezogene Empfehlungen zur Förderung der sozialen Integration

- Der medial viel zitierte "Wertediskurs" bedarf der direkten Interaktionen zwischen den Geflüchteten und der einheimischen Bevölkerung. Demzufolge befürworten wir auf Grundlage der Daten aus unseren Expert/inn/eninterviews dessen permanente Förderung, wobei sich BuddyProgramme als besonders effizient herausgestellt haben. Mittels Face-to-face-Kommunikation kann auch die Akzeptanz der Geflüchteten vor allem in der Bevölkerung ländlicher Räume gefördert werden. Gegenseitiges Kennenlernen in sozialen Interaktionen sowie die Internalisierung von Werten wie der Gender Equality, der Religionsfreiheit oder der individuellen Freiheit der Lebensgestaltung benötigen zur konkreten Umsetzung längere Zeit.

- Ehrenamtlichen kommt in der Arbeit mit Geflüchteten eine tragende Rolle zu. Dies hat sich vor allem in der Phase der Massenzuwanderung der "refugee crisis" in aller Deutlichkeit gezeigt. Zum Zwecke der Aufrechterhaltung der Motivation erwarten sich viele Ehrenamtliche auch einen Respons für ihr Engagement, beispielweise in Form von offizieller Anerkennung, Ehrungen etc. Mögliche Überforderungen der muttersprachlichen Mitarbeiter/innen (Ehrenamtlichen) sollten so gering wie möglich gehalten bzw. vermieden werden. Diese Kategorie von Mitarbeiter/inne/ $\mathrm{n}$ ist nicht automatisch Expert/inn/en gleichzusetzen. 
Viele verfügen über keine fachspezifische Ausbildung, ihre Überforderung ist oftmals geradezu vorprogrammiert. Als Angehörige der migrantischen Communities befinden sie sich darüber hinaus mitunter in einem Zwiespalt, wenn sie unangenehme institutionelle und bürokratische Entscheidungen mitzutragen gezwungen sind.

- Mitarbeiter/innen von NGOs fordern eine generell höhere Wertschätzung der sozialen Arbeit gegenüber. Diese sollte sich auch in einer Anpassung der Gehälter an die steigenden Herausforderungen widerspiegeln.

- Es wird auch mehr aktive und finanzielle Hilfe für Beratungseinrichtungen und Institutionen der Flüchtlingshilfe empfohlen.

- Eine bessere und systematischere Supervision der „practicioners“ leistet einen wesentlichen Beitrag zur Optimierung von deren Output.

\section{Zitate aus den Interviews:}

„R: Also ich glaube primär ein Netzwerk, also vor allem auch ein Netzwerk von Leuten, die schon länger in Österreich wohnen bzw. Deutsch können. ... Also es ist auf jeden Fall wichtig, deswegen haben wir auch diese Initiative, dass man einfach Kontakt hat zur Mehrheitsgesellschaft. Dass man nicht in so Massen-WGs untergebracht ist, wo man keinen Kontakt hat zu den Österreichern. Und dass man auch ein Zuhause hat und ein bisschen Ruhe hat, sein eigenes Leben so zu gestalten und nicht nur auf Überleben aus ist. Da sind halt viele Leute, die in 
diesen Massen-WGs sind, die kommen einfach nicht dazu. Oder es sagen uns viele, dass sie mit Österreichern oder Österreicherinnen zusammenleben wollen, dass sie irgendwie Zugang suchen zu Leuten. Uns schreiben auch immer wieder Leute, dass sie sich ehrenamtlich betätigen wollen, weil sie sich halt irgendwie einen Austausch erhoffen. Deswegen habe ich jetzt auch zuerst Netzwerk gesagt. Weil ich irgendwie das Gefühl habe, dass das dann weiterhilft. Also wenn du ein Netzwerk hast, dann kann dir davon auch jemand sagen: ,Hey, ich weiß einen Job und hätte da an dich gedacht.' oder 'Es gibt die und die Möglichkeiten sich fortzubilden.' Das ist einfach das Einfachste, wenn du Leute kennst, die sich wiederum in dem Land auskennen oder in der Stadt auskennen, dass du da halt Zugang bekommst. Und man braucht halt auf jeden Fall eine gewisse Offenheit, dass man das auch will und dass man diesen Kontakt auch sucht. Es gibt schon auch Leute, die dann irgendwie nichts zu tun haben wollen und dann mit anderen Geflüchteten oder mit anderen Personen aus ihrem Herkunftsland irgendwie abhängen. Und dann gibt es andere Leute, die sich sehr interessieren dafür. Auch für Unterschiede in den Lebensweisen und der Lebenswelt und dann auch so Fragen stellen, wie z.B.: ,Wie ist das jetzt genau? Wie spricht man eine Frau an?'. Da gibt es auf jeden Fall viele unterschiedliche Zugangsweisen oder Offenheit. Und man lernt ja dann auch die Sprache viel einfacher, wenn man Leute kennt, die die Sprache sprechen. Und das ist halt schon etwas, wenn jemand schon gut Deutsch kann, hat er einfach nochmal mehr Möglichkeiten sich auch selbstständig $z u$ bewegen und auch Kontakte zu knüpfen und 
Möglichkeiten zu finden, was er machen kann. " (EXP MR3)

„R: ... und ich finde, es wird auch von den Medien nicht gezeigt, dass man immer noch etwas tun kann. Also es ist irgendwie so, es schlafen keine Leute mehr auf der Straße oder am Westbahnhof, denen geht es jetzt eh gut und fertig. Aber es ist eben auch notwendig, dass die Leute in einen Austausch kommen, dass sie Kontakte knüpfen können usw. Das habe ich auch mit dem Wohlstand gemeint. Man sieht es eben nicht so und wenn dann auch noch die Medien wegschauen oder nicht thematisieren, dass es eigentlich noch genug zu tun gibt, kommt das halt auch nicht so an (Anm.: in der österreichischen Bevölkerung)." (EXP MR3) 


\section{Empfehlungen basierend auf der Teilstudie mit Respondent/inn/en aus Syrien und dem Irak in Wien}

Sabine Bauer-Amin

\section{WoHNUNG}

\section{a. ÜBERGANGSWOHNUNGEN (OHNE PROVISION/KAUTION)}

Für viele Geflüchtete stellt der Übergang von der Grundversorgungseinrichtung zum privaten Wohnungsmarkt eine große Herausforderung dar, die von vielen Schwierigkeiten begleitet ist. Nicht nur ist der Wohnungsmarkt für (vor allem) günstigere Wohnungen beschränkt, zudem ist es schwierig, ohne festes Einkommen (und dessen Nachweis) in den engeren Kreis potentieller Mieter/innen auf dem privaten Wohnungsmarkt zu kommen.

Zusätzlich werden in der Regel hohe Summen an Kaution und Provision für Wohnungen verlangt. Es wurde oft berichtet, dass auch Privatpersonen Zahlungen für das Vermitteln von Wohnungen verlangen, die häufig die Provisionen für professionelle Vermittlungsagenturen übertreffen.

Um diesen Schwierigkeiten entgegenzusteuern und eine weitere Ausnutzung der Vulnerabilität Geflüchteter auf dem Wohnungsmarkt zu verhindern, haben sich Übergangs- und Startwohnungen verschiedener Hilfsorganisationen (z.B. 
Diakonie) als Best-practice-Beispiele herausgebildet. Hierbei werden Wohnungen direkt von der Organisation angemietet. Diese bürgt zudem im Falle von etwaigen Zahlungsrückständen der Mieter/innen.

\section{Zitate aus den Interviews:}

"R: I know a lot of people, who took houses from organisations but I did not. And they got it without paying the deposit. With Caritas and so. This is very good." (S_f_33_SBA_11)

\section{ARBEIT}

\section{a. LeHRALter ANHEBeN}

In vielen Interviews wurde das Faktum angesprochen, dass Personen, die ein bestimmtes Alter überschritten haben, keine Möglichkeiten offenstehen, eine Lehrausbildung zu beginnen. Angesichts der Tatsache, dass viele der Betroffenen noch mehr als 20 Jahre im Arbeitsleben stehen werden, würde sich empfehlen, bei entsprechender Vorbildung bzw. nachgewiesenem Interesse auch Personen älterer Geburtsjahrgänge den Zugang zu Ausbildungen zu erleichtern und dies zu fördern bzw. weitere spezifische Angebote zu entwickeln.

\section{Zitat aus den Interviews:}

" $R$ : And my age has gone beyond the point where I could make an Ausbildung. [At a] maximum of 25 they get it. I crossed that. I exceeded 25 with 10 years. So the topic is hard. From my side for example, it is not important what I want to work, I just want to work." (S(P)_m_35_SBA_3) 


\section{b. ZWISChen-/ SONDERSTUfen für Personen mit LANGER BERUfSERFAHRUNG ABER OHNE LEHRABSCHLUSS}

Für Personen, die bereits über eine lange Berufserfahrung in einem Berufsfeld verfügen, jedoch (noch) nicht ausreichende Sprachkenntnisse aufweisen oder deren Fachwissen nur bedingt mit der beruflichen Alltagspraxis korreliert und deren langjährige Erfahrung daher nicht anerkannt werden kann, empfiehlt sich die Einführung einer qualifikatorischen Zwischenstufe zwischen " angelernt" und „mit Fachausbildung“.

\section{Zitat aus den Interviews:}

"R: I started working at age 10. 10 Jahre.

[...]

$R$ : You could say 40 years, actually.

I: That is a huge experience. Very, very long.

R: Until recently, I have done that. And also now, I want to work here. But wherever I apply here, they tell me that I need to learn German. I tell them "take me to work and I will learn." But they don't. But I would learn during work. I understand that this is Wand, this is Tisch, bisschen, Rolle. This and that. Leiter, Klebebank, Nylon, Karton, malen, Farbe. Whatever they need. Schwarz, gelb, grün." (S_M53_SBA_16)

\section{BILDUNG}

a. ANPASSUNG DER INTEgRATIONSKURSE AN DIE TEILNEHMER/INNEN; EVALUATION 
Die Integrations- und Orientierungskurse wurden von den Teilnehmer/inne/n sehr unterschiedlich bewertet. Ein Teil der Befragten nahm sie als eher überflüssig, nicht hilfreich und nicht den Bedürfnissen entsprechend wahr. Es wäre daher empfehlenswert, die Kurse den Bedürfnissen und Interessen der Teilnehmer/innen anzupassen sowie eine externe Evaluation der Inhalte und der Methoden durchzuführen.

\section{Zitate aus den Interviews:}

"I: And the value classes? Was that good? Did it help you?

$R$ : What do you mean?

I: The ÖIF class?

R: No. Zero.

I: Not at all.

R: Because there was a teacher and he thought he needed to tell us how to speak with a woman. [shakes head].

I: So things you learn as a child.

$R$ : I mean, yes, as a child. We spent the time for nothing there. Senseless. We are not from a forest.

I: Hm. Did he also show you other things like, for example how the e-card works?

R: That I knew already before. So it was really worth nothing to go there. Waste of money for them. I think it takes about one month. And because of this I lost about three months in which I could do a German class, because I missed the beginning. The classes always start together." (S_m_25_SBA_1)

\section{b. WERTEKURSE MÖGLICHST BALD NACH DER ANKUNFT}


Die Werte- und Orientierungskurse wurden am ehesten von Personen mit kurzer Aufenthaltsdauer als nützlich empfunden. Idealerweise sollten sie daher möglichst bald stattfinden. Ab einer Aufenthaltsdauer von einem Jahr wurden sie öfter als nicht mehr hilfreich wahrgenommen.

\section{Zitatbeispiel:}

"RY: We have been here since a year now and we know all of these things. For those, who are new, in their first or second month, this information is really helpful. This is really informative for them but not for us. But maybe we will learn something new, too."

\section{c. Anerkennung von Postmigrationsstress/PTSD/Traumata}

In vielen der Interviews hat sich gezeigt, dass Befragte großem Postmigrationsstress ausgesetzt sind. Dieser entsteht durch limitiertes Wissen über die neue Umgebung, fehlende Sprachkenntnisse, das Einfinden in neue Rollen, Strukturen und Identifikationen sowie die Schwierigkeiten, die mit dem (Wieder)aufbau sozialer Netze verbunden ist. Hinzu kommt, dass viele Geflüchtete vor und auf ihrer Flucht mit Gewalt, Tod, Haft und anderen existentiellen Schwierigkeiten konfrontiert waren, die oftmals nicht ausreichend aufgearbeitet werden konnten. In Österreich kommt neben der Sorge um die Familie, die im Heimatland zurückgeblieben ist, die finanziell prekäre Situation, der Statusverlust, Xenophobieerfahrungen sowie rechtliche Ungewissheit hinzu. Diese Stressfaktoren können oft nicht ausreichend bewältigt werden und führen zu Konzentrationsstörungen, Isolation oder psychosomatischen Störungen. 
Um nachhaltige Initiativen für Geflüchtete gestalten zu können, müssen diese Prozesse und Stressoren sowie mögliche Traumata/PTSD anerkannt und mitgedacht werden.

\section{Zitatbeispiel 1 aus den Interviews:}

" $R$ : My family comes from Ghouta. Have you heard about Ghouta? It is all under bombing, attacks, killings. You have heard about the problems there? When somebody has a background like this and a family under such conditions? Do you think I would have the peace of mind to study when I have to keep up with the news about my family? That is a huge problem. It is problematic for someone like me, in my age specifically. I do not want to give you problems or disturb your head, but it is really hard. Let us continue." (S_m_53_SBA_16)

\section{Zitatbeispiel 2 aus den Interviews:}

" $R$ : We Syrians, three quarters have psychological pressures [or stress]. They have psychological conditions. For sure everyone who has left Syria has something. They have a lot of psychological problems, problems, problems. I am also psychologically stressed myself. Wallahi, I have psychological pressures. It is true, I am here with my children, but the rest of my family are still under bombing. They were bombed yesterday and they were picked up from under the rubble. I can show ou the picture when they were picked up from under the rubble [shows the pictures] This is one thing. And also my son, my son, I don't know if he passed away or if he is good. Therefore, I have psychological stress. Do you know how? I am not able to focus. There is something distracting me. Something is occupying my mind 
here. I have things working in my brain at the same time. I cannot focus. I forget very fast. No, not like this. I cannot forget my family! No! I cannot forget my first-born. In our Arab society, the first-born son, the mother cannot let him go. You can never forget your son. You might have lost your father, your brother, your mother. It is a hard thing, see how? (S_f_48_SBA_13)

\section{d. Sprachiurse strukturieren nach Alter UND VORBILDUNG UND DURCHMISCHEN HINSICHTLICH DER SPRACHGRUPPEN}

Als nicht hilfreich hat sich die Einteilung von Kursen (v.a. Sprachkursen) nach dem Kriterium der Herkunftssprachen erwiesen. Dadurch konnten keine Kontakte außerhalb der Gruppe geschaffen und der Spracherwerb nicht gefördert werden. Von vielen Befragten wurde stattdessen vorgeschlagen, die Kurse hinsichtlich der Herkunftssprachen zu mischen, jedoch hinsichtlich des Alters und des Bildungsgrades der Teilnehmer/innen zu separieren.

\section{Zitatbeispiel 1 aus den Interviews:}

"G: The students are not always all at the same level. Some are really good, some know a bit and others don't understand anything.

$R$ : And everyone speaks Arabic. That is also a problem. We are supposed to speak German throughout the German classes. But if all the people are from Syria or Iraq, of course they only speak Arabic with each other.

$G$ : In Arabic it is very easy to connect to the others. You are from Syria and you are from Iraq, why should I speak German with you? So I speak Arabic. 
$R$ : Yes, if the group is mixed and there is for example one from Afghanistan, one from Poland one from Serbia, we speak German with each other.

G: Yes, this way you have to, because you do not know each other's languages.

$R$ : And that is very important. We always have to speak German so that the teacher understand which level we are at.

I: So, you mean, it would be better if the classes were better mixed?

R: Right." (I_m_27_SBA15)

\section{Zitatbeispiel 2 aus den Interviews:}

" $R$ : My girl, there is a lot of routine here. I, for example, I am 53 years old. But look, the ones above 40, if they cannot work, they will not learn anything. The people enrolled with me in the course, are all old. They just keep looking at their watches. They are not learning anything. They just keep looking at the time and how slow it goes. They just want to get over with the course and go back home. They do not want to learn." (S_53_m_SBA_16)

\section{Zitatbeispiel $\mathbf{3}$ aus den Interviews:}

"And there are different age groups in the same class. There are elderly people with very young people. Almost minors with retirees. This process is very bad to acquire the language. There are other systems. In the language schools in Sweden people stay for eight hours, stay with the teacher, eat with him and only speak in the local language. In Austria it does not work very well. It is about the institute and the teachers. [In English] It is about the criteria. If they have high criteria. In Austria, they have very low criteria for the teachers. [In German] AMS chooses with very little money the cheapest." $\left(S(K) \_m \_30 \_S B A \_21\right.$ 


\section{e. Möglichieiten SChaffen, Österreichische QUALITÄTSMEDIEN KOSTENFREI ZU KONSUMIEREN}

Um den Umgang mit der deutschen Sprache zu fördern, könnten zum einen kurze Lernvideos oder Apps vermehrt eingesetzt werden. Zum anderen wäre es hilfreich, den Sprachlernenden den Zugang zu deutschsprachigen Medien zu erleichtern durch z.B. geförderte Abonnements von Zeitungen/Zeitschriften

oder Unterstützungen/Informationen zu TV und/oder Radio (z.B. zu Onlineangeboten).

\section{Zitatbeispiel aus den Interviews:}

" $R$ : Wallah, routine, routine, routine. If there is a class, I go to class, if there is none, I stay in the house. We eat, we clean the house. There is nothing, not even a TV to watch. I: Oh, so you also cannot watch Austrian TV to hear the language?

R: No, that also needs a bit of money.

I: Yes. So what do you do? Do you read? Do you spend time on the internet?

R: I am on my mobile phone." (S_f_48_SBA_13)

\section{SOZIALE INTEgRATION}

\section{a. FLÜChtLINGE IN VEREINEN INTEGRIEREN}

In vielen der Interviews wurde betont, dass Geflüchtete gerne außerhalb der sozialen Kategorie der "Geflüchteten“ Kontakte knüpfen wollten. Daher mieden viele von ihnen die Hilfsangebote oder Initiativen, die "Geflüchtete" als 
Zielgruppe hatten. Eine Alternative wäre daher die Aufnahme in Vereine, wie etwa Feuerwehren, Fußballvereine, Chöre etc., in welchen Geflüchtete als Vereinsmitglieder außerhalb ihrer Geflüchtetenrolle als gleichwertige Mitglieder wahrgenommen werden könnten.

\section{Zitatbeispiel 1 aus den Interviews:}

"I: And that is really important. So what would be your advice to others who arrive newly in Austria to find contacts?

R: Through hobbies. There are so many clubs in Vienna. People can go there. Whenever someone likes something like music, dancing, sports, you can go there and meet people. Step by step. Yes." (S_m_25_SBA_1)

\section{Zitatbeispiel 2 aus den Interviews:}

"Being at same eye height. To have relationships at the same eye heights. If I am seen the same way as everyone else. I feel good then. And when I am understood in what I say. Especially, in German. Sometimes, one makes these little language mistakes but they have a huge influence on the meaning. I want that people understand me well. I would say these two things." ( $S(K) \_m_{-} 27$ SBA_23)

\section{b. Schaffung Von MöglichKeIten des KontaktS MIT GLEICHALTRIGEN}

In vielen der Interviews wurde angemerkt, dass der meiste Kontakt außerhalb der eigenen Sprachgruppen zu Ehrenamtlichen und Freiwilligen verschiedener Organisationen bestand. In vielen Fällen waren dies 
entweder Student/inn/en oder Pensionist/inn/en, für die der Kontakt primär nicht auf persönlicher Freundschaft basierte, sondern auf ehrenamtlichem Engagement.

Um jedoch mit gleichaltrigen Österreicher/inne/ $\mathrm{n}$ in Kontakt zu treten, fehlte es oft sowohl an finanziellen Mitteln als auch an Informationen, welche Möglichkeiten der Freizeitgestaltung es gibt. Hinzu kam, dass auch strukturelle Hindernisse vorhanden waren, wie etwa die Einlassselektion von Diskotheken.

Best-practice-Beispiele hierfür wären Freiwilligenangebote, in welchen viele Geflüchtete selbst aktiv sein könnten und darüber hinaus auch Kontakte zu anderen Gleichaltrigen herstellen könnten. Alternativen wären die bereits genannten Vereinsaktivitäten.

\section{Zitatbeispiel aus den Interviews:}

"R: Yes. And I want to mention something else. We cannot mingle with people in our age. We can for example not go to a club. They do not let us in because of our face. They think we are thieves, we will cause fights or beat up people. G: This also happens to women. My friend, she is 27 and she wanted to go to a club but they did not let her in.

$R$ : Does she perhaps wear a headscarf?

G: No, no. No headscarf. The security asked for her passport. He saw it was grey and then he did not let her in. I: Why that?

$G$ : Because she is a refugee. That is enough.

$R$ : Many people say, if you want to find friends, you go to the club and get to know people. But we cannot go there.

$G$ : In a club one gets to know women.

R: Women? Yes, women but also men. Both go there. In general, you get to know people there.

I: So where can one go then? 
G: Swimming pool.

R: No, no, one stays at home. There is no place for us. The people in Austria go-.

G: Gym maybe.

R: Gym? No, not to make friends. In the gym, everyone is a foreigner too. You cannot meet Austrians there. That is not useful. I can go there to meet new Arab friends, maybe 5 ." (I_m_27_SBA_15)

\section{FöRdERUNG VON NACHBARSCHAFTS- BZW. HAUsinitiativen}

Auffällig oft wurde der Wunsch genannt, Nachbarschaftsaktivitäten zu fördern. Für viele der Befragten basierte Integration zu einem erheblichen Teil darauf, tragende Beziehungen zu Personen im selben Haus/Viertel aufzubauen. In manchen Fällen haben sich Nachbarschaftsinitiativen bereits ergeben, was sehr zu begrüßen ist.

" $R$ : I have really good neighbours. We live like on a compound. And within it, there is a garden. Every Sunday, we make a Grill in there, barbecue. This way, many neighbours come by. And they sit with us. Once, one

${ }^{5}$ Der Respondent meint, dass er in den Fitnessstudios, in die er geht (oder gehen kann), keine Österreicher/innen trifft. Dafür lernt er dort neue arabischsprachige Freunde kennen. 
neighbour that I do not really know, once came and told me, "You brought life to this place." In this place, no one was making barbecues or so before. She said "You really made something out of this place." So then we invited her for the next time. There are always people coming." (S_f57_SBA28)

Vor allem Nachbarschaftsbeziehungen wurden von Geflüchteten als besonders erstrebenswert erwähnt.

\section{Zitatbeispiel aus den Interviews:}

"R: Even, in our building [homecountry] there were 14 floors. There were more than 120 flats. And there were maybe 4 houses like ours next to each other. We knew them all. Everyone knew everyone. Even if there was someone new, we knew their door before we even saw them. And I miss this. My husband smokes. But he did not smoke in the house. He went outside and smoked with the neighbours. Now, I still send him out in the corridor to smoke but even there he does not meet anyone. Just once.

Here they do not like to see each other. They do not like to eat with each other. They tell us we have to be in contact and integrate. But how? There has to be integration, there has to be integration. We Arabs, we love to integrate, by the way. You have lived in Egypt, so you know that we Arabs all love to do these things all together and always love to talk to everyone and be social. But here, they do not love this integration! They are the ones." ( $S f_{-} 48$ SBA_13)

\section{d. Aktive Trauerarbeit mit Geflüchteten}


Viele der befragten Personen haben in den vergangenen Jahren nicht nur Haus und Besitz, sondern auch Angehörige verloren oder zurückgelassen. Bisher war hierzu wenig bis keine Trauerarbeit möglich. Diese könnte individuell oder kollektiv gestaltet werden und der Verlust sollte öffentlich anerkannt werden.

\section{Zitatbeispiel aus den Interviews}

R: "Me personally, I have lost three of my relatives, a brother, a nephew and another nephew from another sister.

I: May God bless their souls.

R: And I also lost my sister's husband in this devilish game. So four people. One of my sister's husbands lost his house in Aleppo, and went to Idlib. He lost his house in Idlib and went to a Damascene suburb. He recently lost this on in one of Damascus' suburbs. He lost three houses. So he went to rent in Latakia. He lives there now. There are tragedies. There are people who lost their children. Complete families are wiped out. The terrorism that Syria has witnessed, has not been witnessed by any other country in the world. Ever. It is as if whoever is doing this is not human, does not have blood and flesh like us." (S_m_72_SBA_10)

"There is not yet a Syrian mosque and no Kurdish mosque. They do not have the money, they are a poor community. We are one of the poorest communities in Austria. The Turks are richer and the Egyptians are richer and also they are old communities. The Kurdish community is more politically oriented. We have the parties. Every party has a centre. But now we realized that we need a Kurdish progressive mosque. So that people could go there when someone passes away. This is also a problem. We are thinking about it now because 
it is not enough to have only things about culture. There are also other problems." (S(K)_30_m_SBA_21)

\section{WoHLBEFINDEN}

\section{a. Spezielle Programme für Ältere Gefüchtete}

Aus den Interviews ergab sich auch, dass vor allem für ältere Geflüchtete wenige Angebote vorhanden sind. Da sie weder in die Zielgruppe der Maßnahmen zur Arbeitsmarktintegration noch in Initiativen bzw. Angeboten für vulnerable Gruppen, waren sie zu einem großen Teil von Integrationsangeboten ausgeschlossen. Sprachkurse waren auch oft nicht auf die Bedürfnisse und speziellen Herausforderungen älterer Befragter ausgerichtet. Aufgrund der sich verändernden demographischen Zusammensetzung der Geflüchteten in rezenten Jahren würden sich spezielle Angebote für ältere Geflüchtete empfehlen.

\section{Zitatbeispiel 1aus den Interviews}

"Therefore, I suggest this, for elderly people, from all these countries where they came from: all these elderly who came from Syria, Iraq or Afghanistan and so on. One should call them once per week from a special centre with some advice on how to integrate or how to live if they choose to stay. How to address people, how to integrate in society, what you would say in a restaurant, how to act there plus a little bit of entertainment, like good movies and such. So they would get over this spare time. These people over 60, 65 or 70, like me. At least one time per week in a special centre. A person should come and speak in Arabic to give them some advice on how to integrate with the Austrian people. It is about 
food, the Austrian society, traditions, what they eat, their customs, what they prefer here in Austria. Would they prefer to eat in the house or the restaurant? All these cultural things. Also to bring some movies, to show it to these people. Movies of one or two hours. Take this movie for example to take about this; this movie is about this, this movie is about that. This migrant or refugee must feel that there is a little bit of attention given to him. This side of the picture is completely ignored, here in Austria, or Germany or anywhere.

I have not seen a person, even here in humanitarian organizations, who would do that. I haven't seen any of them, not a single one. Although I know they exist. But I haven't seen even one. Where are these organisations that take care of elderlies? I have not seen anyone." (S_m_ 72_SBA_15)

\section{Zitatbeispiel 2 aus den Interviews}

"I would want to have a club or an association where older refugees could meet with Austrians the same age. Maybe this would help to study the language faster. The organisations should help to establish contacts. For the grown-ups." (S_f_48_SBA_13)

\section{b. DRUCK AUS DER TRANISTORISCHEN PHASE NEHMEN}

Der bereits angesprochene Postmigrationsstress ist vor allem in der transitorischen Phase hoch. Gerade in dieser Phase wäre es daher ratsam, keinen Integrationsdruck 
aufzubauen und stattdessen unterstützend zu wirken. Dies erleichtert den Übergang, hilft Ressourcen zur Bewältigung aufzubauen und dient daher längerfristig einer nachhaltigen Integration.

Zitatbeispiel:

"You should not encounter these teenagers in a pejoratively way or look down on them from day one. You should meet them at the same eye height and explain the country to them from there. And show them the country's support and the language. But with professionals. But when you give them many big fields and tell them "you are a refugee and you have to do this and this and this", they cannot cope with that. They will break. [...] Look, if you say this is integration, these are the criteria, these are the values and you have to adapt, then this is one way. Another way is to say, "hey, you came to Austria and you are about to stay a while so try to understand this country and get to know it." And not even use the word adaptation. So, it should not be built on duty. of course, there are some duties so that the system works, basic things. But one should explain them in a way that they understand it as a win-win situation. Not just that to do things in order that the Austrians are fine with them but also that they have a better life in the country. So that they can orient themselves better and understand the country better. Integration yes, but not with a pointer finger and written rules and so. So not "if you do something wrong you get punished!" But "Do you want to have good chances here? Do you want to be part of society? Do you want to feel well in Austria?" Then you can explain that it is necessary to put effort in it, to go and talk to people and to mingle with them, listen to them and be able to also handle critic." $T(K)$ 57_m_SBA_17) 


\section{SChulungen der Betreuer/inNen, UM ÜBERFORDERUNG UND SEKUNDÄRER TRAUMATISIERUNG VORZUBEUGEN}

In Interviews wurden auch Erfahrungen mit Betreuuer/inne/n/Freiwilligen angesprochen, die zeigen, dass Personen mit Situationen überfordert waren. Maßnahmen zur professionellen Schulung von Ehrenamtlichen im Umgang mit bsp. Traumatisierungen, wie dies bereits in einigen Organisationen Usus ist, sind daher sehr begrüßenswert.

\section{Zitatbeispiel aus den Interviews}

"What resulted from the fact that many were not prepared?

R: That many employees were wiped out after six months, almost traumatized themselves. They themselves did not know how to continue. And often they projected their anger on the refugees themselves. And many could not cope with it any more and left [the institution] again." $\left(T(K) \_57 \_m \_S B A \_17\right)$

\section{REPRÄSENTATION}

a. VerantWORTUNGSBEWUSSTER UMGANG DER Medien Mit deR Fluchtthematik 
Ein häufig angesprochenes Thema in den Interviews waren österreichische Medien, wobei vor allem Gratisprintmedien gemeint waren. Geflüchtete erkannten in diesen einen Medienbias, der sich gegen Geflüchtete äußerte, essentialisierend und oft stereotypisierend ausgerichtet war. Daher wurde häufig die Forderung nach einem verantwortungsbewussteren Umgang mit der sensiblen Flüchtlingsthematik und persönlichen Daten verbalisiert.

\section{Zitatbeispiel 1 aus den Interviews}

"R: Yes, definitely. Actually, for me everything went fine. I only had to wait for two months for my asylum recognition. Too fast actually. And everything went well except finding an apartment. That was too hard. Especially, because back then I was not working. And they have always asked for my pay slips. And you know because of Syrian.... Many media here produce propaganda or so. Some have negative prejudices.

I: Hm. Why do you think it is this way with the prejudices?

$R$ : Because of media.

I: Because of media?

$R$ : Yes. Or because of the things that are happening in the world.

I: Do you mean the attacks?

R: Exactly. Attacks or sexual harassment. That as well." (S_m_25_SBA1)

\section{Zitatbeispiel 2 aus den Interviews}

"Unfortunately, now more than 90 percent belief that. "Aha, refugee, and he is, aha, he came and he only wants money, taxes." That is written in the comments that I read 
every day. Zeit im Bild, ORF. I look at the comments. Madness, madness. Now they talk, earlier already, since three years about this shitty IS group. Before that, I have not seen that someone writes against Islam or writes against refugees. Now, all commentaries, look at them, 90 percent are against Islam. They rant against Islam, or Muslims, or religion. They rant against refugees, against Syrians. Sometimes I read comments for Assad and against the refugees. "Do more [indicates beheadings], they need that." Please. Look at the media and what they are doing. That is all because of media. If media was honest and upright, if I was an Austrian and look at media and reality then I cannot write that on Facebook. For sure, I cannot. But if I [switches to Arabic] spread hatred or make people hate, how is it in German? [Switches to German] Hatred! Hatred! And the other as well, hatred. If I was Austrian and would read that, then I think- I hate all refugees. It does not matter if it is a real refugee or a good refugee or so. But I would hate them." (S_m_34_SBA_5)

\section{Zitatbeispiel 3 aus den Interviews:}

"Media should find a solution. Because they destroyed us, honestly. When I open Facebook sometimes, I do not want to mention names, you should see the comments when they say refugees have done this or that. Just look at the comments. I want to tell them, "What do you think of us? We are just people." I have learned the language. I just want to convey a good image to the people. That is it so to say. But sadly, I feel disappointed. I sit and think. One is really in pain over what one reads and finds. It is a lot of difficulties about integration. Sometimes I think about my personal opinion. People here, somebody should educate them. This is really important." (S_m_27_SBA_2) 


\section{b. Monitoring von HASSREDEN}

Oft wurde in den Interviews auf die Hetze gegen Geflüchtete hingewiesen. Es war den Befragten klar, dass diese benutzt wurde. um gezielt falsche Informationen zu verbreiten. Hinzu kommt, dass die Diffamierung Geflüchteter oft einhergeht mit Islamophobie. Dies kann in bezug auf die Ausprägung der Religiosität Geflüchteter einen Gegentrend auslösen, wie das nachfolgende Beispiel zeigt.

\section{Zitatbeispiel 1 aus den Interviews:}

"R: Perhaps they would stop hate-speech about refugees, Islam or anything that is different.

$R$ : There are hate-speeches that exist and they spread wrong

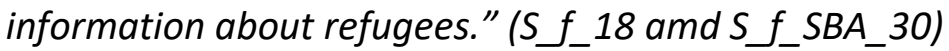

\section{Zitatbeispiel $\mathbf{2}$ aus den Interviews:}

"I know some, who have not been religious at all in Syria. They drank alcohol, they also had girlfriends and they did all kinds of things. But here, they identify with Islam. They have a beard now and pray five times per day. And this radicalisation has happened here in Europe. This radicalisation, well, it is not a radicalisation, but they become more religious. I think this is the fault of Europeans. But this is also, so to say, it is complicated. They all, all the refuges, all the Syrians are confronted with Islam here. And they are judged because they are Muslims. They are denunciated for being Muslims. This scheme did not exist in Syria. We were not classified according to Islam or as Muslims. This classification is something that we do not 
have. And if we are classified and perceived according to our relation to Islam then we have a stronger bond to Islam. Then we identify with Islam. And we become more religious. [...] It is influenced by the media, by the political debate, by the streets, in the classes and not by the mosques. The mosques do not have a big influence, because we do not go there often. They influence the Arabs who are here since a long time. We are still new. If you are told, you are like this all the time, you become like this." (S(K)_m_27_SBA:23)

\section{AnerkenNung Von Und UnterstützUng für EIGENE INITIATIVEN}

In vielen Fällen haben sich bereits eigene Initiativen Geflüchteter entwickelt, die helfen, sich in Österreich zurecht zu finden. Es existiert eine Internetgruppe, die mit ihren mehr als 56.000 Mitgliedern inzwischen weit mehr Personen umfasst, als neuangekommene Syrer/innen, für welche sie ursprünglich ins Leben gerufen worden war. In diesen Gruppen werden Informationen ausgetauscht, Gegenstände verkauft, Probleme und Herausforderungen in Österreich diskutiert und gegenseitige Unterstützung (z.B. Übersetzungen, Informationen zu Ansprechpartner/inne/n) ausgetauscht. Die Administrator/inn/en fungieren dabei als Lots/inn/en, um Falschinformationen zu verhindern. Dies ist oft mit einem hohen Zeitaufwand verbunden. Die Bedeutung dieser Gruppen sowie der Einsatz bestimmter Administrator/inn/en und besonders aktiver und hilfsbereiter Gruppenmitglieder ist bisher nicht sichtbar gemacht worden. Denkbar wäre es auch, Administrator/inn/en mittels spezieller Schulungen zu unterstützen. 
Zudem gibt es auch Offline-Initiativen, in welchen Geflüchtete einander gegenseitig beim Erlernen der Sprache oder der Bewältigung von Alltagsproblemen helfen. Auch hier wäre es denkbar, diese Anstrengungen mehr anzuerkennen und eventuell mit Informationen $\mathrm{zu}$ kostenlos nutzbaren Räumlichkeiten oder Förderungen seitens der Gebietskörperschaften zu unterstützen.

\section{Zitatbeispiel 1 aus den Interviews:}

" $R$ : I am an admin in a group [40:00]. Also in an Arab group. 45.000 members.

I: Wow, that is a huge group.

$R$ : And I, since 2014, I gathered a lot of experience. But my head is for sure destroyed. For sure, insane. A person calls me from Innsbruck, Syrian and asks, "Sorry [R], I have problems with this. How can I do it?" Please.

I: So they all have your number?

R: No, in messenger. Or, when I see it is a good person, I also give out my number, but not to everyone. Only some. And I need to answer. Please, I cannot answer everyone. There are five admins. But some are-, there are two that really help. But helping takes time. From me, from others. It takes a lot of time. My friend told me, "[R], if you have a lot of experience, then work for Caritas or Diakonie. Please." It always takes my time, on Facebook, to help. For example, they need something. There are Syrians, they are like maniacs. Sure, maniacs. They for example ask in this group for the addresses of interface. Please, you can search for this address with google! Just type interface and that is it. It drives me crazy, these maniacs.

I: $\mathrm{Hm}$, it is a really large group. How many questions do you get per day? 
R: I cannot answer that. Sometimes... We are admins. As soon as someone makes a post, I have to click on okay. [...] I have to give an okay for each post as an admin. If the admins do not accept the post, the post is not published.

I: A closed group?

$R$ : Yes, a closed group. And there are many posts. Every day there are 500. But we do not accept all. We only accept help or real questions. For example, there is a man who asks us to allow his post to sell cigarettes, black. Or Shisha or something. Many things. Black, black market. Illegal.

I: Oh, yes, that can be dangerous. And is this group for everyone or for Syrians?

R: No, no. For Syrians. There are also Iraqis. Also Egyptians come. [smiles]

I: Yes, everywhere. [smiles]"

(S_m_34_SBA_5)

\section{Zitatbeispiel 2 aus den Interviews:}

"R: Yes. And we have founded a club.

I: Yes? What is the name of your club?

R: Nadi al-Jalil (Jalil is a depooulated city in Palestine during the war 1947/48; The Galilee forces, Quwat al-Jalil, are a pro-Palestinian militia in Syria). Galileo Verein in German. And there are 45 to 50 members. At the moment we are trying to expand to not just include Palestinians from Homs, but also other Arabs mainly from Syria. But yes, also Austrians. This is what we are trying at the moment. It is not, so far we were not so successful.

I: Good luck with it. And what are you doing in this club?

R: We speak with each other. "What are the problems?" "What did you do?" "What do you want to do?" And we exchange our experiences. The people who speak German, good German, are not so many in this club. Maybe 5 to 6. 
The others are still learning. And the German language is number one of what one has to know or to study. First of all, the German language.

I: So to say it is a club to help each other and to mutually support each other?

$R$ : Exactly, exactly. Some people in this club already found work, even without German. They have connections. And they work. [...] The main idea is to help each other. For example if someone needs something. What help is it? For example someone has a problem in the flat. For example one needs to move from one house to another. There is one that has a problem with a hospital, health issues. I don't know. And we all try to help each other. For example, someone who speaks the language helps someone who does not speak the language. Systems like that, social gatherings, political parties to get together anyone who knows someone. Maybe somebody knows Austrian people and would introduce us to them. This way there would be an advisor for every family who needs an advisor. There are many elderly Austrians who need assistance. Maybe some of them can be also helped by the people [of our club] who are working. We are still at the beginning of the journey in the country. And learning the language, not exclusively for kids."

(S(P)_M35_SBA_3)

\section{d. Reconcilliation während des Aufenthaltes IN ÖSTERREICH}

Vor allem in der rezenten Fluchtmigration haben viele Betroffene Bürgerkriegs- oder bürgerkriegsähnliche Konstellationen erlebt. Die Bürgerkriegsfronten, z.B. in 
Syrien, wurden auch in den Interviews thematisiert. Unabhängig von einem dauerhaften zukünftigen Aufenthalt in Österreich oder einer Rückkehr nach Syrien würde sich der derzeitige Aufenthalt in Österreich für ReconcilliationsMaßnahmen anbieten. Damit kann Spannungen und Konflikten zwischen ehemaligen Bürgerkriegsparteien in Österreich, aber auch darüber hinaus vorgegriffen werden.

\section{Zitatbeispiel aus den Interviews:}

"Politics of course, because Assad is still staying. Of course. Maybe if Assad is away and murdered or so, I will go to the streets and dance. Maybe I will dance naked. Please, only Assad. That creates big problems, big stress for me. Also here in Austria, if I see someone who supports Assad, I get stressed immediately. Crazy. He killed my family. Not just my family, all these Syrians are murdered by him. And then I see a refugee in Austria who supports Assad! Please! Please go to Assad then. Assad is there. Stay there with Assad. We are here as refugees because of the big problems that Assad created for us. Why are you coming with us to Germany or Austria? That is bad for us." (S_m_34_SBA_5)

Diese Maßnahmen sollten von neutralen Organisationen oder Mediationsstellen organisiert werden und möglichst viele Interessenvertreter/innen miteinbeziehen. Hier können auch Schlüsselpersonen aus Communities, sowie sozialen Medien, z.B. Adminisrator/innen, die ohnehin oft in Schlichterrollen in Online-foren geraten, ausgebildet werden.

\section{SONSTIGE}

a. Digitalisierung der Bürokratie UND bessere VERNETZUNG 
Vor allem die Bürokratie in Österreich wurde seitens der Interviewten häufig angesprochen. Dabei wurde bemerkt, dass es in Österreich oft am Austausch verschiedener Behörden untereinander mangelt, was auch an noch nicht entsprechend ausgebauter digitaler Vernetzung liegen kann. Durch eine bessere Vernetzung und eine damit einhergehende Reduktion von Papierdokumenten könnte nicht nur Zeit gespart werden, sondern es wäre auch möglich, umweltfreundlichere Lösungen zu entwickeln.

In diesem Zusammenhang wäre es auch empfehlenswert, einheitliche Systeme der Namensschreibung bzw. des Umgangs mit Namensketten für Österreich zu finden, um Verwechslungen und damit verbundenen Verzögerungen und überflüssigem Arbeitsaufwand vorzubeugen. Durch die Umschreibung aus der arabischen Schrift in die lateinische ergeben sich verschiedene Umschriftsmöglichkeiten. Im Arabischen gibt es beispielweise keine Unterscheidung der Vokale "e" und "i“ oder "O" und " $\mathrm{u}^{\prime \prime}$, jedoch aber drei verschiedene Buchstaben, die in der lateinischen Schrift oft als "h" geschrieben werden.

Zudem werden in vielen arabischsprachigen Ländern Namen nicht in Vor- und Zuname eingeteilt. Vielmehr haben Personen zwar einen Vornamen, jedoch besteht der weitere Namen aus den Vornamen der männlichen Vorfahren, wodurch sich Namensketten und somit verschiedene Möglichkeiten als Nachnamen ergeben. Bisher gibt es in Österreich weder ein konsequentes System zum Umgang mit Namensketten noch zur Umschreibung 
arabischsprachiger Namen. Dadurch verfügen viele Personen über Dokumente mit unterschiedlichen Namen.

\section{Zitatbeispiel aus den Interviews:}

"R: I had the image that Austria was much developed and would get even better and better. Because Austria is an advanced country. And I expect that these problems that I talked about, the paper issues and so, will diminish in the future. People would have it easier in the future." S_m_72_SBA_15

\section{b. Terminvereinbarungen über Social Media}

Für viele Geflüchtete (und Nicht-Geflüchtete) stellen soziale Medien ein alltägliches Kommunikationsmittel dar. Als Alternative zu telefonischen Vereinbarungen bzw. Terminvereinbarungen per E-Mail, die jeweils ein gewisses Know-how bezüglich Sprache und Formulierungen benötigen, könnten Möglichkeiten zur Terminvereinbarung über soziale Medien eine niederschwellige Alternative bieten.

\section{Zitatbeispiel aus den Interviews:}

"R: Naturally. Or if I need something, that I can write in What's App and we make an appointment." (S_m_34_SBA_5) 


\section{c. Diversitätsmanagement ÜBerdenKen}

In den Befragungen zeigt sich oft, dass Personen, die muttersprachlich Arabisch (da Bezug auf das SBA-Sample) sprachen, als Expert/inn/en für Syrien bzw. den Irak galten. Sprachkompetenz allein ist jedoch kein Garant für einen kultursensiblen und diversitätsbewussten Umgang. Gerade Hierarchien, Marginalisierungen, aber auch Dynamiken der unterschiedlichen Migrationsgenerationen werden hierbei ausgeblendet. Daher wäre eine Professionalisierung des Diversitätsmanagements empfehlenswert.

\section{Zitatbeispiel aus den Interviews:}

„R: The instructor was nice but he was stupid. He told us all the time about his political opinion and this was terrible. He supported the FPÖ. I mean-. He himself is Syrian. Okay, he is here since a long time and has the Austrian citizenship, but I mean still, you are still a foreigner, please, what is this! And he told us many of his opinions and they were simply wrong." (S(K)_27_M-SBA_23) 


\section{Wellbeing and Psychological Distress among Refugees of the Syrian and Iraqi Conflicts}

\section{Leonardo Schiocchet}

First, a few quotations from our LODA interviews will be presented to illustrate some of LODA's most important conclusions regarding wellbeing. Then, general remarks will be presented about psychological care in the Arab World and how it relates to the social situation analyzed by LODA. Finally, a few policy recommendations in the realm of wellbeing and psychological care will be presented.

\section{ForCed MigRANTS' EXPRESSIONS OF PSYCHOLOGICAL DistresS}

This section presents the most widely used expressions of disenfranchisement among our interviewees. Disenfranchisement, in turn, must be understood as one of the most general and pressing issues to be remediated though social inclusion policies in cases of forced migration and displacement.

The Arabic term ghurba ${ }^{6}$ is very widespread through in the Middle East, having given origin also to Turkish, Kurdish, and Farsi counterparts:

\footnotetext{
${ }^{6} \mathrm{Al}$-gharib/ al-ghurba (plural) - the foreign/foreigner, this term can further be compared to the German term "Heimweh".
} 
" $R$ : Just the situation of the country [Syria] bothers me. The war that happened there, the people who are still there.

I: It bothers you a lot or not?

R: Indeed. It creates psychological distress [izem nafsyiah]. I try to forget it, but we are followed daily. One's family, friends, his country, his house, I mean, you know how people live in such country, those who did not leave the country ... my work, all of this was cut off from me. This is my mood.

I: Is it right to call that ghurba [longing for home; feeling estranged; feeling out of place; feeling disenfranchised] or not?

R: Indeed, ghurba in all senses of the word. I mean, wherever people were happy there will be ghurba, ghurba for the nation, ghurba for the family, work, friends ... this.

I: Your house, work, everything you had in Syria.

R: Exactly. I mean, you came to Europe, you left your country, you have to make a new life, and how do you do that when you are 50 years old like me, you, your family, friends, you have to make all this again. (S_W_56_LS18)

Here it expresses acute psychological distress caused by forced displacement, and was widely expressed in the LODA interviews. This is a key term because while its roots also relate to "strange" and "stranger", in this form the term evokes "exile", implying suffering stemming from being uprooted from one's perceived place of national belonging, given that by definition a refugee is displaced from a given nation-state. Thus, when the term is expressed in the context of the LODA interviews, it implied disenfranchisement; that is, not only being uprooted, but 
also suffering from not fitting in the new social context in which the refugee is inscribed.

Along with expressions of "ghurba", and reporting that they have felt "psychologically tired" is one of the most common ways my interlocutors expressed depression and trauma:

I: Now, I would like to know a bit more about health. How do you feel in general?

$R$ : There is nothing.

I: Psychologically, I mean.

R: I'm a bit tired [shway t'aban]. Some days there are a bit of work pressure [dghut shughul], so I feel a bit tired psychologically [t'aban shway nafsyaty]. Also because of my family, and the situation in Syria. These kinds of things. (S_M_32_LS19).

"Work pressure" here is one variant of terms like "pressured" or "strangled", or "chocked", which were also widely used by my interviewees to express that a trauma was actively preventing them from living life in its plenitude. "Feeling psychologically tired" or "psychological pressure" has generally meant in the interviews not being able to get out of the house, being unable to interact with others, and in general having lost motivation to live a healthy life - what the WHO defines as wellbeing.

Along with "ghurba", feeling "psychologically tired", "pressured", "strangled" or "chocked", expressions of 
powerlessness also abounded in my interlocutors' expressions of illbeing. Chiefly among them are the Arabic terms qudra and qwa:

\section{I: Can you describe a typical day for you in Austria?}

R: At the moment, my days are a lot the same. And we cannot leap [forward] or improve. Our days, it is a lot about studying the language or looking for job. We are trying our best for that.

Interviewer: And looking for a job takes a lot of time. Thinking about it.

Interviewee: It was hard for me to study the language. And maybe this affected me negatively and cannot start with capacity [qudra].

I: For example, what do you do in the morning or the afternoon? Do you go the course?

$R$ : In the morning, in the first year my kids arrived, I used to take them to school and then go to the course. Sometimes, I attend a course for a group of volunteers. I try to move forward with [my] language [skills]. Honestly, this is what is taking most of my effort. I try to see where there are difficulties and the gates are closed. There is a problem here. In Syria, for example, I enter an office and ask him that I want to sit there to look and learn, here it is hard. First it is hard, and second, accepting the idea itself is hard since you are not part of this society. (S_M_53_LS22).

While they respectively mean primarily capacity and power, they can be used interchangeably as they were in the 
LODA interviews. In practice, expressions of lack or loss of "power" and "capacity" meant that subjects were prevented, by a trauma or psychological distress, to act. Not having the capacity to learn a language, to understand the complex forced migrant legal condition, to go to work, or to interact with people were common expressions of psychological illbeing among my interlocutors.

Most glaring in all three quotations bellow is how acute psychological distress produced by incertitude - one of the most common effects of forced displacement - is reduced by the subject's acceptance as a refugee in a given host country, in this case, Austria.

I: What changed once you got your asylum here in Austria?

R: Many things, I could have a passport, apply for a job, I mean, enough, my residence is here for 5 years and hopefully I will renew it. There are some fears, maybe it doesn't get renewed and we have to return. But now we have security, my mind is at ease [nafsy raha]. Psychologically [nafsiaty] enough, my passport is Austrian, that one they gave, so it changed many things I want. Additionally, also the e-card, I didn't have it with me (S_W_26LS23).

I: What is changed in your life since you got your asylum papers? For example, that you were working, I don't know.

Interviewee: No. I was not working or studying. We were living in the [refugee] camp. A teacher used to come as a volunteer to give us classes. But you were not 
psychologically stable [mustaqar nafsyan] because you were not recognized for asylum. When I got my papers, I felt psychologically stable [staqaret nafsyian] and started to have a help from the 'Sozial' [Austrian Social Welfare System]. Of course, back then my family was in Turkey and they needed to pay the rent and there was no one to support them economically, so I would send [money] to them. So, there was some kind of stability so I would save my family in Turkey and help them (S_M_39_LS7).

I: What changed when you had your asylum claim accepted?

R: All of us in the camp, I mean, we didn't sleep. Even an identity we didn't have the luxury to possess. Do you know? Not rights, or something else, never. These papers you get, you feel that you have value, I mean, you can go, you can travel if you want, you are stimulated [thafaz]. With these papers you can attend courses, you make it [the course] with people, without these papers you can't attend these courses. I mean, it changed a lot of things in our lives, of course (S_M_32_LS3).

Having one's "mind" at ease" or literally "comfortable", or being "psychologically stable", is the opposite of feeling "psychologically tired" and "not having power/capacity" (or motivation) to steer their own lives in the direction they themselves see fit.

Being recognized as a refugee is most often perceived by my interlocutors as the first necessary milestone in

\footnotetext{
7 The Arabic term used here is also a synonym of "spirit", "life", "being", "soul".
} 
regaining control of ones' self. It "stimulates", or motivates the asylum seeker to start over her/his life. Thus, it should be noted that expecting as much motivation to engage in the host country (and participation in social inclusion policies) from asylum seekers as one does from those already recognized as entitled to asylum is unreasonable. Thus, successful social inclusion policies must already target asylum seekers, before deterioration of psychological health becomes harder to handle.

"Suffering from language", or "having difficulty with language", is one of the most widely used expressions of disenfranchisement among my interlocutors:

I: Refugees. What are your biggest challenges in your life here in Austria?

R: You mean, the most difficult thing?

I: Yes.

$R$ : At the moment, language is a big difficulty. And some days at work there are difficulties. But it is going well with work now since you have experience. Language is the most [important]. I will do the Ausbildung now as I have difficulty [ș'awba] with language. I feel people who are being tortured [t'adhyb - in the sense of suffering] the most here are from language (S_M_32_LS19).

The language barrier is seen as acting upon two interrelated levels: a) on a grand scale, it prevents their hosts from getting to know who my interlocutors really are 
(more so than the other way around), which would prevent their representation though stereotypes and prejudice in the media and Austrian social circles. b) Furthermore, the language barrier also halts interpersonal communication, hindering the forced migrants' participation in even the most basic social activities, which in turn prevents them from asserting their social space and integrating to the local social tissue. According to my interlocutors, part of what is preventing many from language learning is the psychological obstructions (i.e. trauma and depression) they must overcome.

The quotation below expresses how psychological distress preventing, for example, language learning, can be eased through social interaction.

I: And were these expectations [in relation to housing] fulfilled?

R: When we first came to Austria, the weather here is suitable. In Norway it is too cold. But here the weather is close to that of Syria. And something else: we know that the Austrians ... when we were living in the camp [Traiskirchen] we were psychologically tired [nafsiat t'abana] and sad a lot [hazinin jidan], but then when we met the Austrian people, to get the residency, our expectations changed in a positive way. Then we started our courses, started to learn German, very fast, after 5 or 6 days we started to understand a bit ... it became preferable, in general. (S_M_32_LS3).

It is important to notice that the interviewee did not understand interacting with Austrians only as a linguistic 
matter, but also one that made her happier, motivated her, "put her mind at ease". Therefore, if one needs to learn German to integrate, one needs to integrate to learn German. The relationship between these two terms is not simply one of cause and consequence, but it is dialectic. Social inclusion and language policies must take place concomitantly and complementarily.

According to our interlocutors, along with the lack of social life and the language barrier, exclusion from the job market is seen one of the most dramatic forms of disenfranchisement in Austria, deepening subjects' psychological distress, as it is expressed in the following quotations:

I: What is positive or negative about searching for work here? And what are your biggest challenges?

R: I cannot tell you what is positive or negative until I go through a challenge. But right now, the biggest challenge is that I cannot live those challenges. I feel that when I pass next to a workshop and see it growing up from sand, to a hole and then a building, is like passing next to a restaurant and smelling your favorite food and you want it badly. You feel there is something pushing you, but you cannot open the door and enter. And this is very difficult. If there is a program like the one that the consultancy or the Ausbildung did. A special one. But I see after the Ausbildung finished no one cared anymore. (S_M_53_LS22).

I: What would you need to feel more comfortable here in the country? And how can Austrian organizations and the Austrian government do, so you feel better here? 
R: I think each one should have a goal in life. You walk towards your goal, and achieve it. This makes me and the society happy, because then you would be effective in it. And you would be positive, and not negative. Right now, I am negative in society. I am not doing anything for it, and this doesn't satisfy me.

I: What are the main steps for you to settle down in Austria?

$R$ : The main step is to work, and then work, and that is what they are telling us. But there is something not being solved (S_M_53_LS22).

Most of our interviewees expressed deep connection between their professions and their individual identity. So, recuperating their professional space is seen as not only a means to guarantee income and the possibility of fulfilling gender and social roles (as providing for the family, in the case of most men or single mothers), but also to regain control of one's life through mitigating identity loss and/or reconfiguration in exile. Therefore, along with policies targeting social inclusion and language learning, policies aiming at professional inclusion are seen by LODA's interviewees as top priority even from the point of view of health and wellbeing.

\section{Psychological Care in the Arab World}

Language and work are perceived by my Arab interviewees in general as the two most effective ways of dealing with depression, and thus inclusion. But not always people are able to learn, especially the elderly. So, most refugees expect that the Austrian authorities and people should take that into consideration and either fix the 
problem by, for example, creating classes just for the elderly, or just letting it go and forfeiting the need for them to learn the language. The perceived way towards inclusion then is through their children, who can be effective members of the Austrian society if only they are granted opportunity. They can still contribute to society by contributing to the effectiveness of their own household, as the elderly can not only for example help with the children (elderly women) but also help to guide the young (now the heads of the households).

Furthermore, in regions of the Arab world ravaged by war and conflict, where psychological distress is rampant, the psychologist is indeed not such an uncommon sight. However, this professional is generally not giving consultation in a psychology clinic as much as it would be the case in Europe, but she is typically hired by an organization such as an NGO or a grassroots relief effort. The professional of the psyche's work is then typically framed not as clinical, but as social work. Having the cause of the distress recognized as stemming not from the individual's own psyche, but from extrinsic social violence, and being the treatment framed as collective and not individual, greatly mitigates the taboos associated with mental health care in most Arab contexts. It redirects the burdens of being singled out as one "crazy" individual, to one more victim of social injustice and strife.

In as much as many of the interviewees presented here do talk significantly about their own psychological distress and sometimes even mention that they are being medically treated, there is much more under the surface, and only slightly more than half of our sample of 60 in-depth interviews among people displaced from the Syrian and Iraqi conflicts actually directly expressed suffering or having 
suffered from any form of psychological distress, including but not limited to depression and/or physical trauma.

As our LODA interviews demonstrate, most Arab refugees would seek medical treatment of mental illnesses in the most extreme cases, but not psychological council by a professional. The LODA interviews overwhelmingly point towards the general understanding that psychological distress should be treated by talking to family or close friends, when not only internally by the individual her or himself. Also, the family structure is often evoked even when the family unit itself is not, as in the case of an Arab woman who suggested bringing elderly Arabs from the Arab world to "guide" younger ones in Austria. This points to the general conclusion that, for the Arab refugees interviewed, mental health is a private issue belonging to the realm of the (individual) self or at least within the family unit whenever possible and within the intimacy of the closest social ties when the family structure is unavailable. Only extreme cases are expected to be treated by a professional and through medication, but even then, some interviewees concluded that mental illnesses cannot be fully treated by medication and ultimately have to be resolved by introspection and selfcontrol. In other words, psychological distress - from psychological fatigue to depression and trauma - is generally seen as having to be solved from within the self, even if its cause is generally accepted as extraneous. However, the interviewees of the LODA sample, as most other Arab refugees among whom one of our LODA researchers, Leonardo Schiocchet, has conducted fieldwork since 2006, seem generally open to taking advice from social workers, or psychologists working as social workers. 


\section{Policy Recommendations on Wellbeing and Psychological}

\section{CARE}

Based on LODA research results and in view of the above, we recommend practical interventions for what the Austrian government and Austrian NGOs in general understand as refugee inclusion (or integration) policies.

1) Social inclusion policies, especially those incentivizing keeping forced migrants socially engaged, should target already asylum seekers, before deterioration of psychological health becomes more deeply embodied in the forced migrant's psyche.

2) In Syria and Iraq, as in the Near East at large, personal identity can be very much tied to profession, especially in the countryside but not only there. For example, the pharmacist of the neighborhood is a well-known figure, and when she/he is impaired from working, this may cause deep psychological distress. Feelings of being unproductive can be overwhelming for Syrians and Iraqis. Therefore, remunerated occupation cannot be treated as only or mainly an issue of financial security, and policy in this regard must favor solutions that keep forced migrants actively engaged as productive members of society.

3) Forced migrants' psychological distress must be framed as a collective issue and treated under the frame of social work. Even when individual psychological consultation and/or therapy must take place, taboos associated with psychological care in 
the Arab world can be avoided by calling the psychologist a social worker.

As a principle, it is more effective to empower the refugees to solve issues on their own as a collectivity as much as possible. Thus, integrating the refugees as a kind of "social workers" and/or creating the framework through which they can organize themselves around their own grassroots civil society institutions to tackle their problems should reduce psychological distress and foster social inclusion within the Austrian context (Comparable solutions could be found in UNRWA refugee camp projects).

4) Fostering social clubs, grassroots civil organizations and social activities can have a very positive effect towards social inclusion. Syrians, and especially Syrian women, are accustomed to organizing themselves into civil society groups (called in Arabic Jama'yat - civic rotation credit and communitarian help association), so it should not be difficult to find leadership and know-how among them. In the Austrian context, initiatives targeting children are suggested as priorities with high potential for success. However, the realms of language learning and job market were by far those expressed by Arab refugees as hiving had the greatest impact in their psychological health. Therefore, earning a living and being able to speak German must be considered the highest policy priorities. 


\section{Integration von Geflüchteten in der Gemeinde St. Andrä-Wördern (NÖ)}

Erhebung in einer strukturstarken, zentrumsnahen Region Österreichs (Teilstudie, empirische Forschung: April bis November 2018)

\section{Andreas Schwarzbauer}

Die Marktgemeinde St. Andrä-Wördern liegt im Bezirk Tulln in Niederösterreich, zirka 20 Kilometer nordwestlich von Wien. Insgesamt leben in den sieben Katastralgemeinden rund 7.500 Personen (inklusive Nebenwohnsitze ca. 10.000). ${ }^{8}$ St. Andrä-Wördern liegt an der S-Bahn-Linie 40 zwischen Wien und Tulln, die Bundeshauptstadt ist daher mit öffentlichen Verkehrsmitteln gut zu erreichen. Im Gegensatz zu vielen anderen Gemeinden in Österreich wächst die Bevölkerung in St. Andrä-Wördern, was sich auch in der Wohnsituation vor Ort niederschlägt und in Interviews immer wieder erwähnt wurde. Im Gemeindegebiet gibt es eine Volksschule und eine Neue Mittelschule, sowie eine Privatschule nach Montessori und eine Musikschule. Außerdem sind auf die verschiedenen Ortschaften insgesamt sechs Kindergärten verteilt. Schulpflichtige Kinder und Jugendliche besuchen somit zum Großteil Bildungseinrichtungen im Ort und sind hier in das

\footnotetext{
${ }^{8}$ Gemeindehomepage St. Andrä-Wördern 1: https://www.staw.at/Unsere_Gemeinde/Unser_Ort/Zahlen_Fakten, abgerufen am 03.12.2018
} 
österreichische Schulsystem integriert. Seit den Gemeinderatswahlen 2015 gibt es auf kommunaler Ebene eine Koalition aus ÖVP (22,18\%), den Grünen (16,38\%) und der Bürgerliste St. Andrä-Wördern (11,43\%). Während in der vorherigen Amtsperiode der Bürgermeister von der SPÖ gestellt wurde, wird die Gemeinde seit drei Jahren von einem ÖVP-Mitglied geleitet. 9,77\% der Wähler/innen hatten bei dieser Wahl die FPÖ gewählt. ${ }^{9}$ Seitens der Gemeinde besteht seit 2015 die Möglichkeit, dass Asylwerber/innen im Rahmen des gesetzlich vorgesehenen Umfangs gemeinnützige Arbeit im Bereich des Bauhofes verrichten können und dafür mit bis zu $116 €$ monatlich entlohnt werden. Außerdem werden mit Ausnahme des Jahres 2018 seit fünf Jahren in Zusammenarbeit mit der Caritas Integrationsgespräche zu verschiedenen in diesem Bereich relevanten Themen durchgeführt.

In der Gemeinde St. Andrä-Wördern gibt es bereits seit mehr als 15 Jahren den Verein "grenzenlos", der sich als örtliche „Willkommensorganisation" versteht und versucht, alteingesessenen und neuzugezogenen Personen Möglichkeiten zur Begegnung und zum Austausch zu eröffnen. In den letzten Jahren waren auch immer stärker geflüchtete Menschen miteingeschlossen, die zusammen mit den anderen die Veranstaltungen wie Koch- oder Tanzabende und das Sommerfest besuchten. Im Rahmen des Vereins wird außerdem der so genannte Solidarfonds

\footnotetext{
${ }^{9}$ Gemeindehomepage St. Andrä-Wördern 2: https://www.staw.at/Politik_Verwaltung/Wahlergebnisse_als_pdf abgerufen am 17.08.2018
} 
gesponsert, woraus Geflüchtete finanziell unterstützt werden können, um zum Beispiel Fahrkarten zu Deutschkursen oder Prüfungsgebühren zu bezahlen. Im Jahr 2015 wurden außerdem zwei weitere zivilgesellschaftliche Initiativen gegründet: „Das Dorf hilft" und „St. AndräWördern hilft". Mitglieder dieser beiden Gruppierungen kümmerten sich vor allem zu Beginn um das Auftreiben von Sachspenden („Das Dorf hilft") oder die Begleitung von Geflüchteten zu verschiedenen Terminen, zum Arzt oder zu diversen Behörden („St. Andrä-Wördern hilft“). Inzwischen hat sich die Situation etwas verändert: Nachdem der Bedarf an Sachspenden zurückgegangen ist, gibt es die Initiative „Das Dorf hilft" in dieser Form nicht mehr. Gemeinsam mit Mitgliedern von "grenzenlos" und engagierten Einzelpersonen wird von "St. Andrä-Wördern hilft" in den Räumlichkeiten der örtlichen Bücherei dreimal in der Woche ein Deutschkurs für Geflüchtete angeboten. Die Kurse werden gut angenommen, auf den Ablauf und die Inhalte werde ich später noch genauer eingehen. Außerdem gibt es in der Gemeinde St. Andrä-Wördern zwei organisierte Unterkünfte für Asylwerber/innen. Das interkulturelle Wohnheim der österreichischen JungArbeiterBewegung (ÖJAB) wurde bis 2018 viele Jahre lang als Quartier für erwachsene Asylwerber/innen mit einer Kapazität von bis zu 65 Personen geführt, bis es im Sommer vorübergehend geschlossen und renoviert wurde. Im Herbst 2018 wurde das Quartier als Unterkunft für unbegleitete minderjährige Flüchtlinge (UMF) wiedereröffnet. Derzeit leben 47 noch nicht volljährige Burschen dort (Stand Nov. 2018). Außerdem betreibt der Verein „menschen.leben“ eine Unterkunft in der Dammstraße.

Im Großen und Ganzen ziehen die Akteur/inn/e/n in der Gemeinde St. Andrä-Wördern an einem Strang und sind an 
einem gelingenden Zusammenleben und an der Integration von Geflüchteten interessiert. Es gibt einen Austausch zwischen Ehrenamtlichen, Quartierbetreibern und der Gemeinde, wobei alle relevanten Bereiche in mindestens ausreichendem Ausmaß abgedeckt werden. Auch aufgrund der langen Tradition zivilgesellschaftlichen Engagements und des Bewusstseins für Diversität in der Gemeinde gelang es, die Herausforderungen der Flüchtlingsbewegungen 2015/16 gut zu bewältigen. Der Großteil des tatsächlichen Integrationsaufwandes wird dabei von engagierten Einzelpersonen oder aus dem Umfeld der drei Initiativen geleistet, die sich mit dem Thema auseinandersetzen. Von der Gemeinde gibt es dafür infrastrukturelle Unterstützung sowie die Möglichkeit für Asylwerber/innen, im Rahmen der gesetzlichen Vorgaben gemeinnützige Arbeit zu leisten. Im Folgenden werde ich bewährte Maßnahmen für gelungene Integration nach Themengebieten geordnet vorstellen.

\section{EHRENAMTLICHES ENGAGEMENT}

- Für lokale Integration wichtig ist das Schaffen von Begegnungen auf Augenhöhe, bei denen die Geflüchteten nicht auf ihren Flüchtlingsstatus beschränkt werden (bei Kochabenden, Tanzveranstaltungen, Festen etc.)

- Vor allem zu Beginn braucht es umfassende Unterstützung für Geflüchtete (bei Arztbesuchen und Behördenwegen, Deutschkursen, Finden von weiteren Bildungsangeboten, Verweisen auf externe Expert/inn/en, zum Beispiel für Rechtsberatung, Jobsuche etc.). Diese Unterstützung findet in St. Andrä-Wördern im Rahmen der Angebote von "grenzenlos" und der Initiativen, aber auch durch eine Art informelles Buddy-System statt. 
- Um gesellschaftliche Teilhabe von Geflüchteten in einem annehmbaren Ausmaß sicherzustellen, braucht es auch finanzielle Unterstützung für die Menschen (zum Beispiel Fahrtkosten zu Kursen, Deutschprüfungen). Dies wird durch den Solidarfonds von "grenzenlos" ermöglicht.

- Ehrenamtliche fungieren in diesem Kontext als Gatekeeper zu Partizipationsmöglichkeiten und als wichtige Ressource im sozialen Netz (Kontakte, Informationen darüber „wie läuft was in Österreich“).

\section{SPRACHKURS IN DER GEMEINDE}

- Es bewährten sich kontinuierlich tätige Lehrer/innen, die die Schüler/innen und deren Lernfortschritte ebenso kennen wie die Lerninhalte der letzten Einheiten.

- Förderlich sind auch kleine Gruppen, die nach dem jeweiligen Niveau der Beherrschung des Deutschen zusammengefasst werden.

- Der Deutschkurs in St. Andrä-Wördern ist mehr als nur bloße Sprachvermittlung: Es werden Geflüchtete in verschiedenen Bereichen unterstützt. Sie können mit Briefen kommen, die sie nicht verstehen, bzw. nachfragen, was sie nun tun müssen. Es besteht eine Vernetzung auch mit anderen Geflüchteten im Ort, Beratungen finden statt und Personen werden an andere Stellen und Kurse weitervermittelt.

- Der niederschwellige Zugang hat zwar Vorteile, bringt aber auch Herausforderungen für die Lehrer/innen mit sich. 
- Die Bedeutung von Alltagskontakten, in denen Deutsch angewendet werden kann, manifestiert sich vor allem darin, dass man Sprachen generell nicht nur theoretisch in einem Kurs, sondern vor allem in der praktischen Anwendung lernt.

\section{GeMEINDE}

- Von Gemeindeseite bewährt hat sich eine eigene Ansprechperson in Form eines Gemeinderates, der im Rahmen des Sozial- und Kulturausschusses für die Integration von Geflüchteten zuständig ist.

- Von der Möglichkeit, dass Asylwerber/innen beim Bauhof der Gemeinde gemeinnützige Tätigkeiten verrichten können, profitieren sowohl die Geflüchteten als auch die Gemeinde. Zusätzlich steigt damit die Akzeptanz von Geflüchteten in der Gemeindebevölkerung.

- Wichtig ist außerdem die Unterstützung des ehrenamtlichen Engagements durch das Bereitstellen von Räumlichkeiten, Kopiermöglichkeiten, aber auch die Finanzierung der Nachmittagsbetreuung für Kinder von geflüchteten Familien in VS und NMS durch die Gemeinde.

- Was die öffentliche Stimmung in der Gemeindebevölkerung Geflüchteten gegenüber anbelangt, so wirkt sich auch die aktive Thematisierung bei den Integrationsgesprächen, in Berichten in der Gemeindezeitung und die Unterstützung der Ehrenamtlichen positiv aus. 


\section{SCHULE:}

- Im Schulbereich stellt vor allem die Übersetzungsthematik eine Herausforderung dar, weil dies ein sensibler Bereich ist und nicht irgendjemand, der mit dem Schulbetrieb oder der Familie nichts zu tun hat, dies übernehmen kann. Hier wäre es wichtig, für die Zukunft Lösungen zu schaffen, um direkt auf den unmittelbaren Bedarf an Übersetzungen im Schulkontext eingehen zu können.

- Kinder können nur maximal zwei Jahre als außerordentliche Schüler/innen geführt werden. Während vor allem aus der VS diesbezüglich von beachtlichen Erfolgen berichtet werden konnte, sind die Unterschiede im Bereich der NMS nur schwer aufzuholen. Das mag zum einen mit dem fortgeschrittenen Alter der Kinder zusammenhängen, aber andererseits auch mit der Verteilung der vorhandenen Ressourcen zur Sprachförderung, die in St. Andrä-Wördern in der VS höher sind. Von beiden Seiten wurde mir jedoch berichtet, dass es für die Kinder und Jugendlichen gut ist, möglichst bald in das österreichische Schulsystem integriert zu werden und möglichst lange dort eingebunden zu sein.

\section{BASISBILDUNG UND PFLICHTSCHULABSCHLUSSKURSE}

Im Rahmen der Basisbildung und des Pflichtschulabschlusses haben sich sowohl homogene als auch heterogene Gruppen als Konzept bewährt. Es kommt auf die jeweilige Umsetzung an, eine allgemeingültige Empfehlung kann nicht gegeben werden. Im Bereich der Basisbildung ist ein bedarfsorientiertes Angebot (auch Dinge wie Verkehrsregeln, Erlagscheine ausfüllen oder Ähnliches im Deutschunterricht behandeln, Informationen zum 
Versicherungssystem) von erheblicher Bedeutung. Zentral ist der Ansatz, den Geflüchteten Dinge beizubringen, die innen helfen, den Alltag in Österreich auf Deutsch zu bewältigen. Bei den Pflichtschulabschlusskursen hat sich herausgestellt, dass die Kursteilnehmer/innen davon profitieren, wenn auch Absolvent/inn/en früherer Kurse in den Unterricht eingebunden werden und Erfahrungsberichte sowie diverse Tipps und Tricks mit den aktuellen Schüler/inne/ $\mathrm{n}$ teilen können. 


\section{Integration von Geflüchteten in der Gemeinde und im Bezirk Oberwart (B)}

Erhebung in einer strukturschwachen, peripheren Region Österreichs

(Teilstudie empirische Forschung: April bis

November 2018)

\section{Hannah Lichtenwagner}

Die Forschung fand von Mai bis einschließlich November 2018 in der südburgenländischen Stadtgemeinde Oberwart statt. Auf der Ortstafel steht auch noch der ungarische Name „Felsőőr". Für die Burgenland-Kroaten heißt die Stadt Borta und auf Romanes Erba. Drei der insgesamt sechs autochthonen, in Österreich anerkannten Volksgruppen sind in Oberwart vertreten. Dass das Burgenland erst seit ca. 100 Jahren ein Teil Österreichs ist und hier viele ethnische Gruppen zusammenkommen, wird in dieser Stadt sichtbar.

Vor 2015 gab es im Bezirk Oberwart schon drei Grundversorgungsquartiere (Redlschlag, Bernstein und Rotenturm a. d. Pinka). Zusätzlich gibt es auch in Stinatz ein großes Quartier, welches zwar zum Bezirk Güssing gehört, aber auch im Einzugsgebiet von Oberwart liegt. Im Rahmen der Grenzöffnung 2015 bot Oberwart 150 Personen in den Messehallen eine Notunterkunft. Später wurden schrittweise mehr Grundversorgungsstellen eingerichtet. In dieser Zeit wurden auch einige Vereine im Bezirk gegründet, wie die "Flüchtlingsinitivative Südburgenland" oder "Begegnung in Pinkafeld“, in denen sich die Zivilgesellschaft 
organisierte und so die Ankunft von Geflüchteten erleichtern wollte. Im Zentrum von Oberwart besteht das sogenannte „Diakonie-Forum“, welches 2015 eröffnet wurde. Das Forum ist nicht nur das Büro der mobilen Betreuer/innen der Diakonie, sondern auch ein Treffpunkt und Aufenthaltsort für Asylwerber/innen und gleichzeitig ein Raum, wo die Begegnung zwischen Asylwerber/inne/n und Österreicher/inne/n forciert wird. Unweit des Forums findet man im Stadtzentrum eine Evangelische Kirche. Diese Kirche und der Gemeindesaal sind wichtige Orte für viele Geflüchtete, um Unterstützung zu finden oder Feste zu feiern. Die Remunerationsarbeit, die zum Großteil Grünlandarbeit auf den drei christlichen Friedhöfen der Stadt umfasst, wird von einem Team der Evangelischen Pfarre seit zwei Jahren organisiert.

Interviews fanden mit Vertreter/inne/n folgender Einrichtungen und Sparten statt: Arbeitgeber/innen, Regionalbetreuung, Beratung, Politik, Vereine, kirchliche Organisationen und mit Geflüchteten selbst. Im Folgenden werden die Interviewpartner/innen kurz skizziert: ein Unternehmer der Metallverarbeitung, der drei Lehrlinge mit Fluchthintergrund ausbildet - dies ist aufgrund der gesamtburgenländischen Zahl an geflüchteten Lehrlingen, nämlich acht, besonders hervorzuheben. Als Vertreter/innen des Sektors Regionalbetreuung wurde eine mobile Beraterin der Diakonie und eine Regionalbetreuerin der Caritas Burgenland befragt sowie eine Vertreterin der Evangelischen Kirche. Weiters wurden Mitarbeiter/innen von Beratungsstellungen, wie der Frauenberatung, der Bildungsberatung Burgenland, des AMS, der Arbeiterkammer und des ABZ Pinkafeld interviewt. Vertreter/innen der Politik waren der Bürgermeister der Stadtgemeinde Oberwart und die Integrationsbeauftragte 
des Landes Burgenland. Auch wurden zivilgesellschaftliche Initiativen und Vereine aufgesucht, die 2015 gegründet wurden, wie die „Flüchtlingsinitiative Südburgenland" und der Verein "SOL“, der mit EU-Mitteln finanziert wird. Weiters wurden acht Interviews mit Geflüchteten aus dem Irak und Syrien durchgeführt.

\section{RAUM FÜR INTEGRATION}

Aus Interviews mit einer Vertreterin der Evangelischen Kirche und mit einem/einer Vertreter/in des Vereins SOL geht hervor, dass ein großer Teil der Arbeitsmarktintegration auch durch informelle Kontakte stattfindet. Persönliche Beziehungen zu ansässigen Personen können oft zur entscheidenden Unterstützung bei der Jobsuche werden. Der Aufbau sozialer Netzwerke innerhalb der Oberwarter Gesellschaft ist daher für geflüchtete Personen eine entscheidende Triebkraft, um für einen Job ausgewählt zu werden. Auch indirekt kann durch eine Verbesserung der Deutschkenntnisse zu einer schnelleren Arbeitsmarktintegration beigetragen werden. Dafür ist es wichtig, dass Begegnungsräume ohne Konsumzwang geschaffen werden, wo Österreicher/innen und Geflüchtete zusammentreffen und Kontakte geknüpft werden können.

Derzeit besteht in Oberwart das Diakonie-Forum, das nachmittags immer wieder für Freizeitaktivitäten und Workshops für Geflüchtete und Österreicher/innen offensteht. Außerdem gibt es die Evangelische Kirche, in deren Gemeinde sich viele Geflüchtete ein Netzwerk aufbauen. Auch aus dem Interview mit einem/einer Vertreter_in des Vereins „Flüchtlingsinitiative Südburgenland" geht hervor, dass Aufenthaltsräume für Geflüchtete, wo diese ihre Zeit gemeinsam verbringen 
können, wichtig sind. So wurden von diesem Verein eine Teestube und ein Nähtreff organisiert. Beim Verein "SOL“ werden die ersten Prozesse des Kennenlernens und gemeinsamen Arbeitens begleitet, da Missverständnisse aufgrund der Sprachbarriere oder kultureller Unterschiede den Integrationsprozess hemmen können.

\section{WOHNEN}

Das leistbare Wohnen ist eine wichtige Grundlage für die Arbeitsmarktintegration. Kautionen, die hinterlegt werden müssen, und Einkommensnachweise stellen bei der Suche nach einer Mietwohnung oft schwer überwindbare Hürden dar. Die OSG - Oberwarter gemeinnützige Bau-, Wohn- und Siedlungsgenossenschaft reg. Gen.mbH kommt hier auf Wohnungssuchende zu und erlaubt, dass beispielsweise eine Kaution in Raten gezahlt wird. So finden Geflüchtete leistbare Wohnungen in der Stadt und einer räumlichen Segregation wird entgegengewirkt. Bei privaten Vermieter/inne/ $\mathrm{n}$ ist das oft schwierig. Die meisten Wohnungen der zum Zeitpunkt der Felderhebung in Oberwart ansässigen, anerkannten Geflüchteten wurden mit der Hilfe der Evangelischen Kirche vermittelt.

\section{MOBILITÄT}

Ein Auto ist für die Zurücklegung des Arbeitswegs insbesondere in einer peripheren ländlichen Region mit unzureichendem Ausbau des öffentlichen Verkehrswesens notwendig. Gerade in Mangelberufen wie Koch/Kellner sind die Arbeitszeiten oft nicht mit den Fahrzeiten der öffentlichen Verkehrsmittel vereinbar. Metallverarbeitende Betriebe befinden sich meist im Gewerbegebiet und dieses ist wiederum nur schwierig mit öffentlichen Verkehrsmitteln zu erreichen. Doch für den Erwerb eines Autos sind Ersparnisse notwendig, die geflüchtete 
Menschen meist nicht haben. Dies stellt eine Hürde für das Eingehen eines Arbeitsverhältnisses in einer ruralen Region dar.

\section{REMUNERATIONSARBEIT}

Die auf den christlichen Friedhöfen in Oberwart geleistete Remunerationsarbeit ist ein Thema, das öffentlich kontroversiell diskutiert wird. Remunerationsarbeit kann rassistischen Aussagen entgegenwirken, weil Geflüchtete hier unter Beweis stellen, dass sie sich nicht in der "sozialen Hängematte" ausruhen wollen, kann aber genauso auch Xenophobie auslösen. Die gemeinnützige Arbeit wird nicht von allen Ortsbewohner/inne/ $\mathrm{n}$ positiv bewertet. So erzeugt das Auftreten von "fremden“ Männern auch Unbehagen bei manchen Friedhofsbesucher/inne/n.

Wichtig erscheint an dieser Stelle, dass ausreichend Maßnahmen ergriffen werden, um die Information über und die Kommunikation mit den Nutznießer/inne/n dieser Arbeiten zu fördern.

\section{FraUen}

Geflüchtete Frauen können von vielen Organisationen nur schwer erreicht werden. Sie sind oftmals allein zuständig für die Pflege und Erziehung der Kinder und haben weniger freie Zeit zur Verfügung als die Männer. Geflüchtete Frauen sehen sich oft wegen fehlender Kinderbetreuungsmöglichkeiten am Besuch von Deutschkursen gehindert und knüpfen auch weniger soziale Kontakte zu Österreicher/inne/n. Somit können sie die erworbenen Sprachkenntnisse auch weniger häufig anwenden. Zudem richtet sich das Angebot der meisten Oberwarter Vereine, in die vom SOL Geflüchtete integriert werden, entweder direkt oder indirekt überwiegend an 
Männer. So gibt es z.B. einen Männergesangsverein oder Sportclubs, die per se keine Angebote für Frauen haben.

\section{ARBEIT WÄHREND DES AsYLVERFAHRENS/AUSBILDUNG}

Der Dienstleistungsscheck wurde 2017 noch kaum genützt. Während in Oberösterreich über 400 Asylwerber/innen auf der Basis des Dienstleistungsschecks gearbeitet haben, waren es im Burgenland nur 14. Das Burgenland bildet hier das absolute Schlusslicht in Österreich. Es kann nur vermutet werden, dass die Öffentlichkeit nicht ausreichend über diese Möglichkeit der Anstellung und der Handhabung informiert worden ist. So gab es im Bezirk Oberwart auch schon eine Anzeige wegen Schwarzarbeit einer geflüchteten Person, welche durch den Dienstleistungsscheck hätte verhindert werden können. Hier ist eine Nachjustierung notwendig.

Im Bereich der Landwirtschaft wird das Angebot der Saisonarbeit von den Landwirt/inn/en genützt: Sie stellen Asylwerber/innen als Erntehelfer/innen und Hilfskräfte ein. Im Bundesländervergleich liegen die Zahlen im Durchschnitt. Hingegen ist die Zahl der Lehrlinge bundesweit die geringste. Dies korreliert allerdings mit dem strukturellen Mangel an offenen Lehrstellen. 


\title{
Integration von Asylwerber/inne/n und Asylberechtigten in den Arbeitsmarkt. Politik und Maßnahmen in Oberösterreich
}

\author{
Erhebung in einer strukturstarken, zentralen \\ Industrieregion Österreichs \\ (Teilstudie empirische Forschung: Mai 2017, \\ Sommer bis Herbst 2018).
}

\section{Sonja Nechansky}

In Linz fanden größtenteils Expert/inn/eninterviews statt. Neben zwei Interviews mit Landesrat Anschober, der im Land Oberösterreich für die Integrationsthemen zuständig ist, wurden weitere Interviews mit Vertreter/inne/n des AMS, der WKO OÖ und der beiden NGOs, Volkshilfe und Caritas, durchgeführt. Des Weiteren erfolgten Interviews mit Asylberechtigten. In Wels wurden neben Interviews mit dem Vizebürgermeister und dem AMS Wels vor allem Interviews mit Asylberechtigten realisiert. In Kremsmünster fanden zwei Interviews statt, eines mit einem Asylwerber in Lehre und das zweite mit der stellvertretenden Leiterin des Ausbildungszentrums, in welchem der Asylwerber sein erstes Lehrjahr verbrachte. Ein Interview mit der Human Ressource-Managerin desselben Unternehmens fand im Vorfeld in Wels statt. Ein Interview erfolgte in Grieskirchen mit dem Bezirkshauptmann von Grieskirchen und Eferding. Er vertritt alle oberösterreichischen Bezirkshauptleute in der Landessteuerungsgruppe. 


\section{SYSTEME DER INTEGRATION - RAUM FÜR INTEGRATION}

Das Netzwerk zur Verbesserung der Integration ist in Oberösterreich außergewöhnlich dicht und weist mehrere Systeme auf, die so in keinem anderen Bundesland zu finden sind. Im Jahr 2015 wurden Steuerungsgruppen gegründet, um in den einzelnen Bezirken möglichst schnell und effizient den Bedarf an Flüchtlingsunterkünften zu decken. Aufgrund des geänderten Bedarfes wurden diese Steuerungsgruppen 2016 auf Landes- wie auch auf Bezirksebene als Integrations-Steuerungsgruppen eingerichtet. Sie wirken als Kommunikations- und Koordinationsdrehscheibe, durch die der Informations- und Kommunikationsfluss zu den Gemeinden, Interessensvertretern, regionalen NGOs und anderen wichtigen Schnittstellenpartnern sichergestellt wird. Durch die Unterteilung in Landes- und Bezirkssteuerungsgruppe ist eine Kommunikation von oben nach unten und umgekehrt somit gesichert. Mitglieder dieser Steuerungsgruppen sind Vertreter/innen der Hilfsorganisationen, des Arbeitsmarktservices, der Wirtschaftskammer, des Berufsförderinstituts, des Landesschulrates sowie Mitarbeiter/innen der jeweiligen Bezirkshauptmannschaften bzw. der Landesregierung vertreten durch Landesrat Anschober. Vorrangig behandeln diese Steuerungsgruppen die Themenbereiche Bildung und Arbeit.

Als ein weiteres wichtiges Bindeglied $z u$ den Gemeinden fungiert dabei das Regionale Kompetenzzentrum für Integration und Diversität (ReKI). ReKIs unterstützen Gemeinden bei der aktiven Gestaltung des Zusammenlebens von Einheimischen und Personen mit Migrationshintergrund. Sie dienen als erste Anlaufstelle für 
Gemeinden bei allen Fragen bezüglich der Integration von Zugewanderten. Dabei begleiten und moderieren die ReKls Entwicklungsprozesse vor Ort. Des Weiteren beraten und informieren sie bezüglich jeglicher Neuerungen im Integrationsbereich und fördern somit die Vernetzung aller Akteur/inn/e/n in der Region und unterstützen dabei den Austausch unter den Beteiligten.

Trotz der unterschiedlichen Netzwerke und Institutionen ist es für Geflüchtete manchmal nicht klar, welche Kompetenzen und Informationsmöglichkeiten die einzelnen Netzwerkpartner anbieten. Neben dem Aufbau von Vertrauen in die jeweiligen Institutionen betonten Asylberechtigte wie wichtig der Kontakt zu Österreicher/inne/n sei. Es ist außerordentlich herausfordernd, sich ein soziales Umfeld aufzubauen. Vor allem die Situation in Wels wurde als ausgesprochen schwierig beschrieben, da die einzelnen Herkunftsgruppen alle in sich geschlossen sind. Hier fehlt es an Begegnungsräumen zwischen Geflüchteten und Österreicher/inne/n, die notwendig sind, um gezielt den Kontakt zueinander herzustellen. Gerade für die Arbeitssuche sind Eigeninitiative und der Kontakt zur Zivilgesellschaft besonders wichtig und zeitigt auch die meisten Erfolge.

\section{MOBILITÄT}

In Oberösterreich sind die Asylquartiere relativ klein und dezentral eingerichtet, was eine gute Integration in den Gemeinden ermöglicht. Voraussetzung dafür stellt eine leistbare Mobilität dar, um Kurse zu Sprach- und anderem Qualifikationserwerb auch erreichen zu können. Daher fordert das Land Oberösterreich von der Bundesregierung 
denselben Zugang zu gestützten Öffi-Tickets wie für Oberösterreicher/innen ein (durch Lehrlings- oder Jugendtickets). Gerade für jene Geflüchteten, die in periphereren Gegenden wohnen, ist dies ein großes Hindernis, während jene, die im urbanen Raum leben, das Thema Mobilität überhaupt nicht ansprechen.

\section{WOHNUNG/ WOHNRAUM}

Der Zuzug in urbane Ballungsräume ermöglicht einen leichteren Zugang zum Arbeitsmarkt, Informationen über Wohnmöglichkeiten sind allerdings schwer zugänglich. Der derzeitige Wohnungsmarkt in Österreich ist durch ein hohes Preisniveau charakterisiert und es ist allgemein nicht einfach, eine Wohnung zu finden. Durch die Kürzung der Mindestsicherung, die erst im November 2018 vom EuGH gekippt wurde, hat sich die Lage in Oberösterreich weiter verschärft. Während im Sommer und Herbst 2015 die Aufnahmekapazität für Asylwerber/innen gänzlich erschöpft gewesen war, haben sich die Umstände in Bezug auf die Grundversorgungsquartiere inzwischen wieder „normalisiert“. Die Situation anerkannter Flüchtlinge ist indes prekär (Zschiedrich 2016: 4). Nach dem Erhalt des positiven Asylbescheids verlieren sie den Grundversorgungsanspruch und müssen innerhalb einer viermonatigen Übergangsfrist in eine eigene Wohnung ziehen. Subsidiär Schutzberechtigte dürfen noch ein Jahr im Grundversorgungsquartier bleiben (Land Oberösterreich 2017: o.S.).

Viele anerkannte Flüchtlinge ziehen in die urbanen Ballungsräume, weil sie sich dort einen leichteren Zugang zum Arbeitsmarkt erhoffen. Aufgrund der hohen Nachfrage nach Mietwohnungen in den Städten sind die Preise 
gestiegen und Wohnungen werden teuer untervermietet, oft auch in Form von Wohngemeinschaften, wo für ein Bett 250 Euro bezahlt werden muss. Der Zugang zum geförderten Wohnungsmarkt ist streng nach (fünfjähriger) Aufenthaltsdauer in dem jeweiligen Bundesland reglementiert. Da Asylberechtigte, sobald sie den positiven Bescheid erhalten haben, in keinen Statistiken mehr aufscheinen, ist es schwer zu sagen, wie viele von ihnen in Oberösterreich geblieben sind und wie viele Asylberechtigte von kleineren Gemeinden in die urbanen Räume, wie Linz und Wels, umgezogen sind.

\section{BEDARFSORIENTIERTE MindeSTSICHERUNG}

Der Betrag der Mindestsicherung wurde im Juli 2016 für Asylberechtigte mit einer befristeten Aufenthaltsberechtigung und für subsidiär Schutzberechtigte gekürzt. Sie ist in eine Basisleistung und in einen Steigerungsbeitrag gegliedert, wobei der Steigerungsbeitrag (155€ monatlich) nur dann ausgezahlt wird, wenn die vereinbarten Maßnahmen zur Integration im Rahmen der „Integrationserklärung“ eingehalten werden. Ansonsten kommt es zu stufenweisen Kürzungen bzw. einer gänzlichen Einstellung dieser Zahlung. Die gesamte Leistungshöhe beträgt für alleinstehende, erwachsene Personen außerhalb von organisierten Quartieren $560 €$ pro Monat (AK 2017: 3 f.). 
Im März

Landesverwaltungsgericht eine Beschwerde eines subsidiär Schutzberechtigten und gelangte zu der Entscheidung, dass die reduzierte Mindestsicherung für befristete Asylberechtigte nicht gegen EU-Recht verstößt (OÖ Nachrichten 2018). Im November 2018 kippte der Europäische Gerichtshof die oberösterreichische Mindestsicherung mit der Begründung, dass das EU-Recht einer nationalen Regelung entgegensteht, die vorsieht, Flüchtlingen mit befristetem Aufenthaltsrecht geringere Sozialleistungen zu geben als österreichischen Staatsangehörigen (Die Presse 2018). Inwieweit sich diese neuerliche Änderung auf die Wohn- und somit auch auf die Arbeitsmarktintegration auswirkt, konnte im Zuge der Forschung noch nicht festgestellt werden, da die Entscheidung zu aktuell ist.

\section{LEHRE FÜR AsYLWERBER UNTER 25}

Ab 2012 war es per Erlass Asylwerber/inne/n unter 25 Jahren gestattet, während des Asylverfahrens eine Lehrausbildung in einem Mangelberuf zu absolvieren. Voraussetzung war eine Beschäftigungsbewilligung des AMS. Dieser Erlass wurde im September 2018 aufgehoben, obwohl bundesweit über 1000 junge Geflüchtete in Lehrberufen beschäftigt waren. Der größte Anteil davon war in Oberösterreich mit 359 Personen, dementsprechend groß war die Mobilisierung in Oberösterreich um die Lehre attraktiver und sicherer für Asylwerber/innen zu machen. Ziel der Aktion "Ausbildung statt Abschiebung" von Landesrat Anschober war, dass Asylwerber in Lehre für die Dauer ihrer Ausbildungszeit nicht abgeschoben werden, ähnlich wie im Falle des 3+2 Modells in Deutschland. 


\section{Integration von Geflüchteten in den Bildungssektor}

Erhebung in einer strukturschwachen Region in Niederösterreich

(Teilstudie empirische Forschung: Mai - Juli, Oktober - November2018)

\section{Sandra Punz}

Die Erhebung wurde in drei Gemeinden im Mostviertel (Rabenstein, Kirchberg an der Pielach, Frankenfels) durchgeführt, welche mit dem Auto ca. eine halbe Stunde von St. Pölten bzw. eine Stunde von Wien entfernt sind. Sie liegen nicht direkt an der Westbahnstrecke, sind jedoch durch eine Bahnlinie (Mariazellerbahn) mit St. Pölten verbunden. Die drei Gemeinden haben zwischen 2000 und 3200 (Kirchberg an der Pielach) Einwohner und sind einander bezüglich der politischen Rahmenbedingungen sehr ähnlich. Bei der letzten Gemeinderatswahl 2015 erreichte die ÖVP-nahe Liste jeweils zwischen 68 und 70 Prozent, die SPÖ kam auf 18 bis 24 Prozent und die FPÖ auf 6 bis 15 Prozent der Wählerstimmen. Insgesamt wurden 19 Interviews geführt, davon wurden drei als Gruppeninterviews mit jeweils zweimal zwei und einmal drei Personen geführt (Anzahl der interviewten Personen: 23) (siehe Anhang 1 ).

Für die Region lässt sich die Problemstellung bezüglich der Geflüchteten folgendermaßen umreißen: Aufgrund langer Wartezeiten im Asylverfahren wohnen die Asylwerber/innen teilweise länger als 1,5 Jahre in den 
Grundversorgungsquartieren der Gemeinden, die als Selbstversorgerunterkünfte geführt werden. Die Quartiergeber erhalten vom Land monatlich einen um den Tagessatz für Verpflegung (ca. 6 Euro/Person) herabgesetzten Betrag für die Unterbringung (= ca. 15 Euro/Person), dafür sind die Asylwerber/innen selbst für die eigene Verpflegung verantwortlich. Zusätzlich stehen ihnen in der Grundversorgung weitere Leistungen, wie etwa Krankenversicherung sowie Bekleidungshilfe, zur Verfügung. Darüber hinaus haben sie ein Recht auf Information und Beratung. Während dieser Zeit haben sie keinen freien Zugang zum Arbeitsmarkt und Menschen außerhalb des Pflichtschulalters können Bildungsangebote kaum nutzen.

Der niederösterreichische Landesrat für Integration und Veranstaltungswesen, Gottfried Waldhäusl, welcher seit März 2018 dieses Amt bekleidet, sprach sich gegen eine „Integration ab dem ersten Tag“ aus; davon ausgenommen wären Kinder im schulpflichtigen Alter. Das Bezirksblatt Tulln veröffentlichte am 25. Juni 2018 folgendes Statement des Landesrates: „Erst wenn der Asylbescheid positiv ist, dann werden Maßnahmen gesetzt: ,Mit mir wird es keine Integration von Anfang an geben, das ist gegen den Hausverstand', so der Landesrat. In der Vergangenheit sei es oftmals so gewesen, dass geflüchtete Personen bereits in der Lehre, bei der Feuerwehr, ... tätig waren. Dann kam der negative Asylbescheid und vor der Abschiebung haben alle gepoltert, dass die Person bleiben sollte. ,Damit macht man aber eine Tür auf, die gar nicht geöffnet werden sollte. “" (URL 1) 
Vor diesem Hintergrund stellt sich die Frage, in welcher Form Integration (lokal aber auch in den Bildungssektor) möglich ist und wie verschiedene Akteur/inn/e/n mit der Situation umgehen. Im Laufe der Erhebung habe ich folgende Forschungsfrage(n) formuliert: Wie bekommen geflüchtete Menschen mit afghanischer, irakischer oder syrischer Staatsbürgerschaft, die nach 2015 in die Gemeinden A, B und C gekommen sind, Zugang zu Bildung?

- Welche Strukturen der Flüchtlingsbetreuung sind in der Region vorhanden?

- Welche Bildungsangebote gibt es in der Region für nicht anerkannte Flüchtlinge? Welche weiteren Zugangsvoraussetzungen müssen erfüllt werden?

- Welche Herausforderungen werden von Personen, die im Bereich der Erwachsenenbildung für Geflüchtete arbeiten, für eine erfolgreiche Integration identifiziert? Welche Hürden nehmen die Geflüchteten selbst wahr? Welche Erwartungshaltungen gibt es auf beiden Seiten?

- Welche Rolle spielt das ehrenamtliche Engagement für die lokale Integration sowie im Speziellen, wenn es um den Bildungszugang sowie den Erfolg beim Deutschlernen geht?

\section{Darstellung des Datenmaterials \& Policy-Empfehlungen}

Die Integration von geflüchteten Kindern in die Pflichtschule ist gesetzlich festgeschrieben. Demnach müssen Kinder altersgerecht mit einem Spielraum von +/- 
einem Jahr eingestuft werden. Das bedeutet, dass beispielsweise ein/e 14-jährige/r Geflüchtete/r mindestens in die 3. Klasse NMS eingestuft werden muss, selbst wenn keinerlei Deutschkenntnisse vorhanden sind und teilweise auch im Herkunftsland keine Schule besucht hat. Auch wenn diese Vorgaben aus pädagogischen und sozialpsychologischen Gesichtspunkten völlig nachvollziehbar sind, stehen die Direktor/inn/en und Pädagog/inn/en vor der Herausforderung, dieses Kind einerseits entsprechend sprachlich aber auch in allen anderen Fächern zu fördern sowie in die bereits bestehende Klassengemeinschaft zu integrieren. Darüber hinaus sieht das österreichische Schulgesetz vor, außerordentliche Schüler/innen nur zwei Jahre unter diesem Status zu führen - danach soll bzw. muss das Kind in allen Fächern benotet werden können. Dies ist hauptsächlich mit zusätzlichen Ressourcen zu bewerkstelligen, welche aufgrund der doch eher geringen Zahl von geflüchteten Kinder im Untersuchungsfeld in der Regel nicht von staatlicher Seite zur Verfügung gestellt werden. Wie diese Herausforderung bewältigt wird, ist je nach Schulstandort unterschiedlich.

In meiner Erhebung zeigte sich, dass die Schulen einen jeweils unterschiedlichen Modus gefunden hatten, wobei alle auf ehrenamtliches Engagement angewiesen sind entweder von "schulfremden" Personen, pensionierten Lehrkräften oder intern, indem Lehrer/innen in ihren Freistunden die Kinder aus dem Unterricht herausnahmen, um sie individuell zu fördern. Eine NMS konnte auch eine Förderlehrkraft lukrieren. 
Trotz aller Hürden haben alle befragten Direktor/inn/en, die Förderlehrerin und pensionierten Lehrkräfte bestätigt, dass die tatsächliche Integration doch schnell vonstattengeht. Die Integration von erwachsenen Asylwerber/inne/ $\mathrm{n}$ in den Bildungssektor gestaltet sich komplett anders und ist um einiges schwieriger, weil es keine Pflichtausbildung gibt. Erst mit dem positiven Bescheid zur Gewährung von Asyl oder subsidiärem Schutz unterschreiben Asylberechtigte oder subsidiär Schutzberechtigte eine Integrationserklärung, welche den Staat Österreich dazu verpflichtet, Deutschkurse sowie Werte- und Orientierungskurse anzubieten, wobei die Abwicklung dieser Maßnahmen durch den ÖIF erfolgt, die teils mit langen Wartezeiten oder Unterbrechungen zwischen den Kursen verbunden sind.

Ein weiteres Beispiel ist der Pflichtschulabschlusskurs am WIFI. Hierbei sind die Zugangsbeschränkungen etwas strikter. Der/die Bewerber/in muss einen fachlichen Einstufungstest absolvieren und Deutschkenntnisse auf Niveau A2 laut GERS vorweisen können. Sind diese Kriterien erfüllt, stellt sich auch hier die Frage der Mobilität. Vordergründig scheitert es allerdings meist an den zu geringen Sprachkenntnissen. Ansonsten ist dieses Angebot eine gute Möglichkeit, einen Pflichtschulabschluss zu erlangen, wenn das Pflichtschulalter schon überschritten wurde, um darauf aufbauend eine Lehre machen zu können.

Die überwiegende Mehrheit der erwachsenen Geflüchteten in den drei Gemeinden hat derzeit keinen Zugang zu öffentlich geförderten Bildungsangeboten bzw. Deutschkursen. Selbst das ehrenamtliche 
Deutschkursangebot, das es ab 2015 in den Gemeinden gab, wurde aufgrund der geringen Teilnehmerzahl eingestellt bzw. gab es ab dem ersten Halbjahr 2018 ein Deutschkursangebot von der Firma MENTOR in Kooperation mit der Diakonie, welches vom Bundesland finanziert wurde. Dieses dezentrale Kurskonzept sah vor, dass Asylwerber/innen mit hoher Anerkennungswahrscheinlichkeit, die in der Region wohnhaft waren, die Stufen A0-A2 laut GERS absolvieren können. Dazu wurden DaF/DaZ-ausgebildete Lehrpersonen angestellt bzw. Zugtickets organisiert, um an den Sprachkursen teilnehmen zu können.

- Professionelle Asylbewerber/innen
Deutschkurse

mit

für Anerkennungswahrscheinlichkeit im ländlichen Raum flächendeckend sicherstellen: Hierbei kann ein dezentrales Kurskonzept ressourcensparend sein, indem die Lehrperson in die Gemeinde kommt. Die Wartezeit während des Verfahrens kann somit sinnvoll genutzt werde. Nach Erhalt des positiven Asylbescheides können die Menschen schneller in den Arbeitsmarkt integriert werden.

- Ausbildung/Schulung des Lehrpersonals: Wichtig ist hierbei eine DaF/DaZ-Ausbildung, regelmäßige Supervision und spezifische Schulungen für den Umgang mit (traumatisierten) Menschen durch Flucht und Krieg in Gruppensettings (z. B.: Wie gehe ich damit um, wenn eine Person während des Unterrichts oder in der Pause von tragischen Ereignissen/Erlebnissen auf der Flucht oder im Herkunftsland berichtet?). 
Lehrer/innen erzählten, dass sie mit der daraus entstehenden Gruppendynamik teilweise überfordert waren und die Menschen wieder ,in den Unterricht zu holen" bzw. selbst mit diesen Berichten umzugehen.

Empathie für Geflüchtete stellte sich als ein wichtiger Punkt für die Eignung als Trainer/in für Menschen mit Fluchtbiografie heraus.

- Aufbau und Ablauf der Deutschkurse: Wichtig sind Kleingruppen von max. 10-15 Personen des gleichen Levels und dass der Kurs mehrmals wöchentlich bzw. täglich stattfinden kann. Eine externe Prüfung, bei der ein Zertifikat (z. B. ÖSD) erworben werden kann, scheint sich positiv auf die Motivation zum Lernen auszuwirken.

Das Arbeiten mit Büchern, die auf die aktuelle Lebenssituation von Geflüchteten zugeschnitten sind, wurde als wichtig erachtet. Generell wird ein praxisbezogener Unterricht sehr begrüßt. Wichtig ist es auch, einen geeigneten Raum zur Verfügung zu haben. Eine DaF-Lehrerin, die in die Gemeinde kam, um täglich $A 0$ und $A 1$ zu unterrichten, berichtete, dass z. B. die Tafel improvisiert werden musste.

- Ehrenamtliches Engagement in professionelle Strukturen einbinden: Ehrenamtliches Engagement im Bereich der Vermittlung von Deutsch stellte sich seit 2015 als eine wichtige Ressource heraus. Ein innovativer Ansatz kann sein, ehrenamtliches Engagement in professionelle Strukturen einzubinden. Generell wurden ihre Aktivitäten auch von den Ehrenamtlichen selbst als "Notlösung" 
bezeichnet, weil es an Angeboten für Geflüchtete gemangelt hat. Es besteht die Überzeugung, dass die sprachliche Ausbildung hauptsächlich seitens gut ausgebildeter Lehrpersonen (DaF/DaZ) getragen werden muss. Der unverbindliche Charakter von ehrenamtlichen Deutschkursen führt auch dazu, dass die Geflüchteten den Unterricht nicht so ernst nehmen. Die unterschiedliche Qualität der einzelnen, durch verschiedene Ehrenamtliche präsentierten Unterrichtseinheiten garantiert oft wenig Kontinuität und Fortschritt. Für Ehrenamtliche ist es auch schwer, alle Levels in einem Kurs zu unterrichten und sie beklagen, dass sich dies aus verschiedenen Gründen leider auch nicht anders organisieren lässt. Selbst oder gerade um das Level A0/A1 zu unterrichten, braucht es Ausbildung, denn anfangs müssen Kenntnisse der Sprachstruktur vermittelt werden. Die Alphabetisierung von Menschen, die nicht in ihrer Erstsprache alphabetisiert sind, ist besonders schwierig.

- Unterstützung für Schulen anbieten, auch wenn nur wenige geflüchtete Kinder den Schulstandort besuchen. Direktor/inn/en fühlten sich tendenziell alleine gelassen und haben auf Eigeninitiative (externe) Ressourcen geschöpft und geeignetes Material für die DaF/DaZ-Ausbildung organisiert. Dabei wurde auch der Wunsch nach einem Abbau der starren Bürokratie geäußert (z. B. eine flexiblere Jahresplanung aufgrund der hohen Fluktuation von asylbewerbenden Kindern während des Schuljahres) und mehr Transparenz der vorhandenen 
Förderangebote verlangt. Auch im Bereich der präventiven schulpsychologischen Betreuung und der Bereitstellung zusätzlicher Pädagog/inn/en bei mehrtägigen Exkursionen (z. B. Skikurs) wurden Bedarfe geäußert. Die Ressourcen für Deutschförderklassen und -kurse wurden sehr begrüßt. 


\section{Diasporische Gemeinschaften und ihr zivilgesellschaftliches Engagement}

Policy recommendations auf Basis von Interviews mit Geflüchteten aus dem Irak

\section{Maria Six-Hohenbalken}

Bis dato wurde in der Transnationalismus- und Diasporaforschung die Rolle der einzelnen Communities selten im Hinblick auf das generelle zivilgesellschaftliche Engagement in den Aufnahmeländern diskutiert. Eine kritische Auseinandersetzung, die sowohl die Integrationsleistungen der diasporischen Gemeinschaften beleuchtet, wie auch die Nachteile einer solchen (oft informell entstandenen) Integrationsdynamik diskutiert, fehlt in vielen Forschungsarbeiten.

In Österreich entbehrt ein solches Vorhaben einer Grundlagenforschung, um detailliertere Einschätzungen treffen zu können. Die nachfolgenden Überlegungen basieren auf den im LODA- Projekt erhobenen Daten und wurden hier selektiv auf Basis der Interviews mit Geflüchteten aus dem Irak ausgewertet. In einigen Bereichen wird auch auf Daten, die in einem von ROR_n durchgeführten Pilotprojekt (2015-2016) gewonnen wurden, genommen.

Der Fokus auf den Irak ist auch deshalb schlüssig, weil es seit den 1970er-Jahren vermehrt Flucht- und Migrationsbewegungen aus dem Irak nach Österreich gegeben hat und seitdem einige diasporische Gemeinschaften entstanden sind. Diese sind nach wie vor durch ethnische und/oder religiöse Zugehörigkeit oder die 
Herkunftsgesellschaft geprägt. Arabischsprechende, sunnitische irakische Staatsbürger/innen haben je nach politischer Orientierung bzw. Nähe zum Saddam- Regime eigene Netzwerke gegründet - es gab kaum Interaktionen zwischen Regimekritiker/inn/en und -befürworter/inne/n. Schiitische, arabischsprachige Iraker/innen, Kurd/inn/en und Yezid/inn/en aus dem Irak sowie Angehörige von christlichen Gemeinschaften gründeten in den letzten vier Jahrzehnten eigene diasporische Netzwerke, die unterschiedliche Formen von Institutionalisierungen aufweisen (Kulturzentrum, Religiöse Zentren). Die zweite Generation steht bereits im Berufsleben, eine dritte Generation ist im Aufwachsen. Gerade in den Erstkontakten (auf Bahnhöfen, Asylzentren) waren Personen aus diesen Communities überaus wichtige Ersthelfer/innen, Übersetzer/innen und "Gatekeeper". Manch eine/r der ersten und zweiten Generation verfügt über eine einschlägige Ausbildung oder übt eine Tätigkeit im sozialen und Integrationsbereich aus; viele wissen aus eigener (Flucht)erfahrung über die Bedeutung der diasporischen Netzwerke.

Die Einbindung diasporischer Communities in die Integrationsarbeit weist sowohl auf theoretischer wie auch praktischer Ebene eine Reihe von Falltüren auf: Eine Einund Zuordnung der Geflüchteten zu bestehenden diasporischen Gemeinschaften müsste die Heterogenität ausreichend berücksichtigen, die Belastungen einer Integrationsarbeit können diasporische Gemeinschaften auf Dauer nicht tragen. Sicherlich bieten ethnische oder diasporische Netzwerke praktische Informationen, spezifisches Wissen und Zugänge zu Ressourcen, beispielsweise auf dem Wohnungs- und Arbeitsmarkt. Es muss aber die emotionale und psychosoziale Ebene mit 
bedacht werden: Solche Netzwerke können in der Bewältigung der Fluchterfahrungen eine wichtige Rolle spielen, andererseits sind solche Aufgaben für die bereits lange in Österreich Sesshaften eine extreme Belastung und können eigene nicht bewältigte Erfahrungen evozieren. Die über Jahrzehnte entstandenen Gräben zwischen den verschiedenen Communities sowie auch Repressionen, politische Gewalt oder einfach finanzielle Abhängigkeiten spielen eine wichtige Rolle (siehe Clifford 1994; Crisp 1998).

Zu Beginn der Fluchtbewegungen (2015-2016) haben sich arabisch-, kurdisch- oder persischsprachige Freiwillige vermehrt an den weitverzweigten zivilgesellschaftlichen Hilfsprojekten beteiligt bzw. in einzelnen NGOs beschäftigt. Auch haben diasporische Gemeinschaften eine Reihe von Hilfsmaßnahmen ihrerseits gestartet; die LODA-bezogenen Erhebungen lassen erkennen, dass diese Entwicklungen in den letzten zwei Jahren rückläufig sind. Nach wie vor sind Mitglieder diasporischer Gemeinschaften als Professionist/inn/en in verschiedenen integrationsbezogenen und sozialen Berufen tätig - deren Expertisen müssten in den integrationspolitischen Maßnahmen gesondert diskutiert werden (s.u.). Weiterhin sind diasporische Communities wichtige Informationsquellen und Gatekeeper für Menschen auf der Flucht, wie auch Akteure in der Entwicklung von spezifischen Integrationsmaßnahmen (ein besonderes Beispiel hierin ist die demographisch große und für die Religionsgemeinschaft überaus bedeutsame yezidische Diaspora in Deutschland; siehe Blume 2016).

Geflüchtete aus dem Irak haben (im Vergleich zu jenen aus Syrien oder Afghanistan) in Österreich meist nur ein subsidiäres Bleiberecht erhalten und eher seltener einen unbefristeten Asylstatus. Aufgrund der graduellen 
Verbesserung der Situation im Heimatland - abhängig von der Herkunftsregion und ethnischen oder religiösen Zugehörigkeit - war eine Rückkehr in den letzten beiden Jahren wiederum teilweise möglich (Stand August 2019). Mehr als die Hälfte der irakischen Respondent/inn/en aus der Pilotstudie 2015/16 sind wieder zurückgekehrt.

Geflüchtete aus dem Irak, deren Asylansuchen positiv beschieden wurden bzw. deren Status gesichert ist, suchen oft ganz bewusst soziale Kontakte mit Österreicher/inne/n, um beispielsweise die deutsche Sprache mehr zu praktizieren.

An den nachfolgenden Interviewauszügen wird deutlich, dass das Interesse, sich in die bestehenden diasporischen Gemeinschaften einzubringen, sehr gering ist.

" $R$ : In relation to your place of residence how important is it to for you to have people from the same religion as yours in the same country as yours? And that's there are for example mosques or cultural associations or your community? (...) For me these things are not important. You are human and I am also human. This is important for people who are very conservative so they are afraid of others who are different or people who are afraid in general. We call them "qalqa", people who put a shield on." (I_m_22_LS1)

Die Intention wie auch die Erwartungshaltung eines 24jährigen Informanten lautet folgendermaßen:

"I: Who are your most important social contacts? As for example countrymen, Austrians, migrants from others countries

etc.

R: my friends from Iraq they are my close friends, honestly until now I don't have any Austrian friend, I've been here since 3 years and I don't have any Austrian friends 
R: But you want to have one? Interviewee: yeah sure because this will help me to improve my language, to get to know the country, and to integrate well. But it's really difficult thing, I tried a lot through friend or colleague in Ausbildung or at the University, because I see that they are conservative and isolated, and it's not in my nature to go to someone and ask him to be my friends, when someone comes to me he's welcome I directly consider him as a friends, but I avoid the person who (don't feel comfortable with me or who averse from me ..) because I hate the arrogance, we're all human being and we have to be humble, also it shouldn't be classes in the society so that thing bother me actually, because at the end we're one society I mean I have other friends from another nationalities (Romania, Turkey) but my close friends are Iraqi." (I_m_24_SBA)

Aus der nachfolgenden Passage geht auch hervor, dass manche versuchen, mit der Vergangenheit abzuschließen. Jene, die sich anfangs interessiert an einer Interaktion mit den verschiedenen Gemeinschaften in Österreich zeigten, ist die Komplexität der politischen Beziehungen im Nahen Osten oft zu anstrengend, um eine vertrauensvolle Bekanntschaft oder Freundschaft entstehen zu lassen.

"R: But they [Iraqis] have their own way of thinking. I don't integrate with them here. (...) For example I was assistant director in Iraq, and that was a good position. But when I came here I started from the zero point. (...). I used to live in the same flat with a very famous refugee, he is famous around the world. He was a designer, and have got a lot fashion's prizes, and been a guest in many famous channel. And he was living with me, he and I started from the beginning together, from the zero point. He was also a director in the Ministry of Culture. But he said that he want 
to start a new life here, Iraq is a past for him now. "(I_m_29, SB 24)

Im Vergleich zu den Geflüchteten aus Syrien muss für die Iraker/innen auch noch hervorgehoben werden, dass sie aus einem Land kommen, das in den letzten vier Jahrzehnten eine Reihe von Kriegen (Irak-Iran-Krieg, Kuwait etc.) bzw. bürgerkriegsähnlichen Krisen (Kurdistan) und Ausnahmezuständen erlebt hat. Junge Menschen sind daher eher daran interessiert, mit dieser gewaltvollen Geschichte und Sozialisation abzuschließen und ganz neu wieder anzufangen sowie ihr Leben in Österreich aufzubauen. Andererseits scheint es schwierig zu sein, aus den Netzwerken, die Geflüchtete unter sich aufgebaut haben, herauszukommen und Kontakte mit Österreicher/inne/n zu schließen, so wie das zwei junge Männer (R,F) aus dem Irak im Folgenden erklären:

"R: I want to finish B1 and then go to Linz

I: Why Linz?

R: I might have a chance there. It might be better for me. There are companies that might hire me, and their AMS is much more accurate than here.

I: Do you know Linz well? Do you have contacts there?

$R$ : Yes, I have contacts there.

I: Do you like the city there?

R: I like Linz. It is a nice place. I can't say that they are less racist but they are much calmer, according to what I saw. 
I: And you said that at the moment you only have contact with people from Iraq, right?

R: No, not just Iraqis, mainly Arabs.

RR: Mostly they are Arabs. Syrians, Iraqis

I: From where do you know them?

R: I mainly got to know them through the courses and through common friends." (I_m_30, I_m_27_SBA)

Offensichtlich ist, dass die Kontakte, die zwischen den Arabischsprechenden geschlossen werden, für die beiden Befragten nicht jene sozialen Kontakte sind, die sie sich wünschen oder erwarten:

"RR: (...) But this does not help with anything. This is not beneficial for me. We go there from time to time [Arab restaurants]. Whenever we crave something Iraqi or Syrian. And then we also talk to the people.

R: But it does not give me anything of value. It did not make my life any better."

Im Bereich der systemischen Integration (vor allem Wohnen und Arbeiten) und angesichts der Knappheit von leistbarem Wohnraum in Wien dürften sich bald nach 2015 illegale Strukturen herausgebildet haben, deren Protagonisten von der Not der Geflüchteten finanziell profitierten:

"R: For example, there is a lot of Polish people a lot of people from migrant background using this kind of crisis situation to their advantage. So those putting the most burden on the refugees are the migrants. They try to do business to try to 
make your brother or sister or cousin or someone who comes to pay as much as possible because they don't know the language. They made lots of money (...) And they were in the same situation that we are now. But when they go to little bit better then when you come you have to pay a security deposit and you have to sign a contract and for the furniture and you also have to pay a commission. So, I know for example that a lady is renting a place then or a room a house a flat and I can rent it to you and then I show them pictures and then I say you have to pay me $\$ 3,000$ for no reason whatsoever. And it just got higher and higher it reached 5,000. For example, my friend yesterday she got an apartment of one bedroom and the commission she had to pay was 5000 euros. And she had to consider that. She's a single woman, alone with her daughter 5 years old. And she had so much shit in her life you cannot imagine. So then when I hear from somebody that situation that they have to pay $\$ 5,000$ I say what? How does it work this women for example she will have to borrow money? She is actually doing that she's taking money from everyone we know she takes 400 euros from one 400 from the other and so on. Family friends or anyone who can help and she will go through this so she can live in a place." (I_m_22_LS1)

Obwohl die Befragten viel weniger soziale Kontakte mit Österreicher/inne/n aufbauen konnten als sie sich wünschten, setzten sie in diese Kontakte mehr Vertrauen als in jene, die mit Personen aus dem eigenen Herkunftsland geschlossen wurden oder mit Personen mit anderem Migrationshintergrund. Ein Beispiel dafür ist die Entstehung eines Systems, das die Geflüchteten aus ihren Herkunftsländern kennen, wie das des "Wasta“. Ein Wasta ist ein Vermittler, ein „Ermöglicher", ein „Türenöffner", d.h. eine Person, über die man Zugang zu Institutionen oder 
Entscheidungsträgern erlangt. Die Person „Wasta“ verlangt dafür eine monetäre Entschädigung.

"I: Some people use the refugees. Or some refugees and migrants themselves use other refugees because they're profitable. This is really disturbing me. And it doesn't make sense. Interviewer: Is this similar to the "wasta" [person of power that informally or illegally intervenes as mediator/connection] in the Arab world?

R: Yes I was really surprised to see that even in Europe you can make use of a wasta. It is really important. Here, an Austrian wasta is better. In Austria, there is also wasta. That was a revelation for us. When you asked me earlier if I expected anything about Austria or Europe I didn't say it then but what they really expected is that there is no wasta here. This was in my imagination but the reality was very different. And I thought that the government would be more organized. Maybe they are organized in other matters but in what relates to the refugees it's just chaos. Really chaos. I was shocked. It took a while for me to understand this. I'm telling you because it really happens in front of my eyes." (I_m_22_LS1)

"I: (...) How relevant support was your network of co-ethnic, co-religious or co-national persons concerning accommodation?

R: You mean living with people from same religion or Arabs? I: No, Arabs or Muslims or I don't know. Maybe it is not important for you.

$R$ : It is not important for me, not all persons or opinions are the same. It is difficult to find a person that has same as your own ideas." (I_m_30 LS8) 
Es kann festgestellt werden, dass es nach den ersten Orientierungsphasen bzw. in Phasen, in welchen sich die Geflüchteten in einem (rechtlich) unsichere Bereich befanden, kaum Alternativen dazu gibt, als sich auf die diasporischen Netzwerke der Herkunftsgesellschaft zu beziehen. Einige Respondent/inn/en meinten, dass sie gerade von diesen Graubereichen in der strukturellen Integration (Vermittlung von Wohnungen, „Wasta“-System) besonders schockiert waren und sich viel mehr Kontakte zur Aufnahmegesellschaft und eine Professionalisierung der jeweiligen Integrationsfelder wünschten.

Im Vergleich zur Situation in den 1980er-Jahren, als besonders aus dem Irak viele Menschen nach Europa geflüchtet waren und auch Österreich zahlreiche geflüchtete Kurd/inn/en aufgenommen hatte, sind die Vorzeichen heute sehr unterschiedlich zu jenen von vor 30 Jahren. Das Sprachkursangebot ist viel umfangreicher und die Geflüchteten können sich - verglichen mit den 1980erund 1990er-Jahren - schon relativ bald auf Deutsch ausdrücken. Damals wurden auch die diasporischen Organisationen verstärkt mit Integrationsaufgaben betraut (Sozialbetreuung, Wohnraumbeschaffung etc.). Ob dies eine adäquate Maßnahme war, sei dahingestellt, Faktum ist aber, dass aus diesen Beauftragungen eine Reihe von Professionist/inn/en hervorgegangen sind, die in der Folge Ausbildungen im Sozialbereich absolvierten und bis heute in der professionellen Betreuung tätig sind. Auch haben sich die diasporischen Organisationen durch diese Beauftragungen verändert und konnten so beständigere Vereins- bzw. Organisationsstrukturen aufbauen.

Abseits der zahlreichen jungen Personen, mit denen Interviews geführt wurden, kann man auch eine Schicht von Geflüchteten ausmachen, die den Integrationsvorgaben 
(Sprach- und Wertekurse, Ausbildungsmöglichkeiten) nicht in der beabsichtigten Form nachkommen können. Darunter fallen beispielsweise Personen, die aufgrund ihres fortgeschrittenen Alters es nie schaffen werden, auf dem Arbeitsmarkt Fuß zu fassen, des Weiteren auch Personen mit eingeschränkter Gesundheit oder all jene, die ohne familiären Hintergrund in Össterreich gelandet sind und besondere Bedürfnisse aufweisen. Für all jene sind die diasporischen Organisationen ein zentraler Angelpunkt, um ein Sozialleben aufzubauen, um Austausch zu pflegen und auch eine gewisse emotionale Ansprache zu haben. Gerade diese Bereiche können nur sehr schwer von staatlichen Integrationsinstitutionen abgedeckt werden bzw. gibt es bis dato auch wenig Einblick in und Verständnis für deren spezifische Situationen.

\section{Policy Recommendations}

.) Würdigung und Sichtbarmachung des zivilgesellschaftlichen Engagements, das von diasporischen Gemeinschaften geleistet wird

.) Gerade diasporische Gruppen oder Institutionen leisten wesentliche Beiträge in der Unterstützung von besonders vulnerablen Gruppen, die nicht in die staatlichen Integrationsschienen aufgenommen werden können. Solche Bereiche müssen ausgemacht und auch besonders unterstützt werden, u.a. in Form materieller Leistungen.

.) Die staatlichen Unterstützungen diasporischer Gemeinschaften in ihren Integrationsmaßnahmen müssen mit besonderer Umsicht (und speziellem Wissen) erfolgen, da kompetitive Beziehungen zwischen einzelnen diasporischen Organisationen resultieren können. 
.) Wenn es gruppenzentrierte Unterstützungen geben soll, dann braucht es ein Mindestmaß an Standards, die dabei eingehalten werden müssen.

.) Die Förderung von Gatekeepers in Form eines bewussten Diversitätsmanagements beispielsweise im Ausbildungsbereich (Sozialarbeit, Gemeinwesenarbeit) ermöglicht auf lange Sicht eine dialogische Kooperation und die Einbindung jener Integrationskompetenzen, die Mitglieder diasporischer Gemeinschaften mitbringen.

.) Es muss ein Bewusstsein dafür geschaffen werden, dass die Unterstützungen von/durch diasporische Gemeinschaften auch ein hohes Maß an Vulnerabilität für die diversen Akteure impliziert. Diese bzw. deren Eltern haben vielleicht selbst Fluchtbiographien aufzuweisen und sind gerade auch deshalb besonders exponiert und bedürfen besonderer Unterstützung (Supervision etc.).

.) Diasporische Organisationen oder Institutionen können ihrerseits auch die Kontakte mit der Residenzgesellschaft ermöglichen oder erleichtern. Die Einbindung und Förderung dieser Institutionen soll daher auf jeden Fall dialogzentriert und integrationsfokussierend sein. 


\section{LITERATUR}

Aigner, Anita (2016). Wohnraum für Flüchtlinge II. Über (Un)Zugänglichkeiten, gute und böse Subwohnungsmärkte, asyl aktuell 3, 9-17.

Aigner, Anita (2018). Housing entry pathways of refugees in Vienna, a city of social housing, Housing Studies (DOI: 10.1080/02673037.2018.1485882).

AK (Arbeiterkammer) (2017) Bedarfsorientierte Mindestsicherung in Österreich. Antworten auf die wichtigsten Fragen. Arbeiterkammer Oö. URL: https://media.arbeiterkammer.at/ooe/publikationen/a rbeitundrecht/F 2017 Mindestsicherung OOE.pdf [Zugriff: 27.11.2018].

Alam, Hila-Nawa (2008). Afghanische MigrantInnen in Österreich: empirische Untersuchung zu Integration, Repatriierung und der Partizipation am Wiederaufbau des Heimatlandes Wien, Univ., Dipl.-Arb. (UB Wien: http://ubdata.univie.ac.at/AC0661482; accessed 23-122018).

AMS (12-01-2016). Asylberechtigte auf Jobsuche: Kompetenzcheck-Ergebnisse und Maßnahmen zur Integration in den Arbeitsmarkt [http://ams.streaming.at/20160112; accessed 08-022019].

Arbeitplus (Soziale Unternehmen Österreich) (02-102017). Flucht und integration in den Arbeitsmarkt. Arbeitplus-Themenpapier.

[https://arbeitplus.at/wordpress/wp- 
content/uploads/2017/07/ThemenpapierFlucht-201710.pdf ; accessed 20-12-2018].

Bauer, Isabella (10-07-2017). Unterbringung von Flüchtlingen in deutschen Kommunen:

Konfliktmediation und lokale Beteiligung, State-ofResearch Papier 10, IMIS/bicc [https://flucht-forschungtransfer.de/wp-content/uploads/2017/05/FFT SoR-

10 Bauer Unterbringung Konflikte-Mediation 25-07-

2017.pdf; accessed 08-10-2019].

Biffl, Gudrun, et al. (2012). Anerkennung ausländischer Qualifikationen und informeller Kompetenzen in Österreich. In: Schriftenreihe Migration und Globalisierung. Krems: Donau-Universität Krems. Department für Migration und Globalisierung.

Blume, Michael (2016). Das Baden-Württemberger "Sonderkontingent"für besonders Schutzbedürftige aus dem Nordirak. Mit Ausblicken zur Zukunft des Êzîdentums. In: Brizić, K.; Grond, A.; Oztovics, C.; Schmidinger, T.; Six-Hohenbalken, M.: Şingal 2014: Der Angriff des "Islamischen Staates", der Genozid an den Êzîlî und die Folgen. Wiener Jahrbuch für Kurdische Studien. Wien: Ceasarpress, 61-72.

Bruckner, Regina (13-10-2018). 40 Prozent der Wiener Unternehmer haben Migrationshintergrund - Der STANDARD, $\mathrm{S}$. 28.

http://Derstandard.at/2000089238859/40-Prozentder-Wiener-Unternehmer-habenMigrationshintergrund; accessed 12-12-2018]. 
Buber-Ennser, Isabella, Judith Kohlenberger, Bernhard Rengs, Zakarya Al Zalak, Anne Goujon, Erich Striessnig, Michaela Potančoková, Richard Gisser, Maria Rita Testa, Wolfgang Lutz (September 23, 2016), Human Capital, Values, and Attitudes of Persons Seeking Refuge in Austria in 2015, PLOS ONE [https://doi.org/10.1371/journal.pone.0163481;

https://journals.plos.org/plosone/article?id=10.1371/jo urnal.pone.0163481; accessed 29-12-2018].

Cheung, Sin Yi and Phillimore, Jenny 2013: Social networks, social capital and refugee integration, Research Report for Nuffield Foundation April 2013. University of Birmingham.

(http://www.nuffieldfoundation.org/sites/default/files/ files/Nuffield\%20Refugee\%20Exec\%20Summary\%20fin al.pdf; accessed 23-12-2018).

Chiswick, Barry R. and Paul W. Miller (2015), International Migration and the Economics of Language, in: B.R. Chiswick und P.W. Miller (eds.), Handbook of the Economics of International Migration, Vol. 1, NorthHolland, Amsterdam, 211-269.

Clifford, James (1994): Diasporas, in: Cultural Anthropology 9 (3), 302-338.

Crisp, Jeff (1998). Policy changes of the new diasporas: migrant networks and their impact on asylum flos and regimes, WPTC 99-05.

Deutscher Landkreistag, Berlin (Hg.) (o.J.). Integration von Flüchtlingen in ländlichen Räumen. Strategische Leitlinien

und

Best

Practices 
[http://www.landkreistag.de/images/stories/themen/F luechtlinge/161130\%20(DLT)\%20Integration\%20von\%2 OFI\%C3\%BCchtlingen\%20in\%20l\%C3\%A4ndlichen\%20R \%C3\%A4umen.pdf; accessed 10-12-2018].

Dialogforum der Donau-Universität Krems (Hg.) (Sept. 17-20 2018). Dialog zwischen Wissenschaft, Politik und Praxis. Good Practice in Österreich [http://www.dialogforum-integration.at/goodpractice/; accessed 17-12-2018].

Die Presse (2018): EuGH kippt Kürzung der Mindestsicherung für Asylberechtigte in Oberösterreich. Online. 21.11.2018. URL: https://diepresse.com/home/innenpolitik/5533648/Eu GH-kippt-Kuerzung-der-Mindestsicherung-fuerAsylberechtigte-in-OOe [Zugriff: 27.01.2018].

Dustmann, Christian and Albrecht Glitz (2011), Migration and Education, in: E.A. Hanushek, St. Machin und L. Wößmann (eds.), Handbook of the Economics of Education, Vol. 4, North-Holland, Amsterdam, 327-439.

Esser, Hartmut (2001). Integration und ethnische Schichtung. Arbeitspapier 40. Mannheim (http://www.mzes.unimannheim.de/publications/wp/wp-40.pdf; accessed 29-12-2018).

Egger, Eva (Okt. 2013). Integration von EinwohnerInnen mit Migrationshintergrund auf kommunaler Ebene: Maßnahmen für ein Zusammenleben in Vielfalt am Beispiel der Gemeinde Albersdorf-Prebuch, Masterarbeit an der Karl-Franzens-Universität Graz 
[https://online.uni-

graz.at/kfu online/wbAbs.showThesis?pThesis Nr=4537 2\&pOrgNr=\&pPers Nr=53983; accessed 17-12-2018].

EUROCITIES (ed.) (2016). EUROCITIES, Social Affairs. Refugee Reception and Integration in Cities (https://ec.europa.eu/futurium/sites/futurium/files/eu rocities-refugees-report.pdf; accessed 23-12-2018).

Fetz, Kristin (2011). Strukturelle Diskriminierung und Unterdrückung von anerkannten Flüchtlingen am österreichischen Arbeitsmarkt und das Handlungsfeld Sozialer Arbeit auf Basis Struktureller Sozialer Arbeit, BA, "Soziale Arbeit", Management Center Innsbruck, June 2011 (http://goo.gl/X6ccl8; accessed 23-12-2018).

Fóti, Klara and Andrea Fromm (2016). Approaches to the labour market integration of refugees and asylum seekers. Eurofound Research Report [ https://www.netzwerk-

iq.de/fileadmin/Redaktion/Downloads/Fachstelle Ein wanderung/Fachgespr\%C3\%A4che/Studie Eurofound LMI of Refugees and Asylum Seekers.pdf; accessed 23-12-2018].

Frey, V. (2011). Recht auf Wohnen. Zugang zu MigrantInnen und ethnischen Minderheiten zu öffentlichemWohnraum in Österreich [Legal study, part of the PROGESS project 'Equality in Housing'] (Wien) (http://www.bawo.at/fileadmin/user upload/public/D okumente/Publikationen/Berichte Studien/Europa/Eq uality in Housing/rechtliche Studie Equality In Hous ing.pdf; accessed 04-12-2018). 
Gatterbauer, Marlies (2019). Die lange Dauer der Flucht - Das Ankommen erleichtern durch die Begleitung des Arbeitsmarktservice bis zur Arbeitsmarktintegration, in: J. Kohlbacher \& M. six-Hohenbalken (Hg.). Die lange Dauer der Flucht - Analysen aus Wissenschaft und Praxis. ISR-Forschungsbericht 49. Wien: Verlag der ÖAW, 131-142.

Hammerschmied, Anastasia (2017): Asylberechtigte in Wien: Tausend Euro für ein Zimmer. Der Standard. Online. 14.11.2017.

URL: https://derstandard.at/2000067112603/Asylberechtigt e-in-Wien-Tausend-Euro-fuer-ein-Zimmer [Zugriff: 27.11.2018].

Hosner, Roland (ICMPD), Irina Vana (ZSI) und Golschan Khun Jush (ICMPD), Projektleitung: Veronika Bilger (Nov. 2017). Integrationsmaßnahmen und Arbeitsmarkterfolg von Flüchtlingen und subsidiär Schutzberechtigen in Österreich Forschungsbericht des FIMAS-Projekts. Wien: ICMPD/ZSI.

[https://www.asyl.at/adincludes/dld.php?datei=112.10 .ma,fimas bericht final.pdf ; accessed 25-12-2018].

International Organization for Migration (IOM) (Hg.) (2122-2017). Abschlusskonferenz zum IOM Projekt "Integration in den Gemeinden“, Konferenzbericht, 21.11.2017, 09:30-17:00 Uhr, Fleming's Conference Hotel, Neubaugürtel 26-28, 1070 Wien [www.iomvienna.at/sites/default/files/Admin4all Konf erenzbericht.pdf; accessed 03-12-2018]. 
Käfer, Patricia (22-08-2016). Videodolmetschen wenn der Arzt kommt, Die Presse, [https://diepresse.com/home/alpbach/5072495/Video dolmetschen-wenn-der-Arzt-kommt ; accessed 12-122018].

Karakayali, Serhat (unter Mitarbeit von Mareike Heller) (Juni 2018). Ehrenamtliches Engagement für Geflüchtete in Deutschland. State-of-Research-papier 09, IMIS \& bicc [https://flucht-forschungtransfer.de/wp-content/uploads/.../SoR-09-

Karakayali.pdf ; accessed 23-12-2018].

Kohlbacher, Josef, Rasuly-Paleczek, Gabriele, Hackl, Andreas und Bauer, Sabine (2017). Wertehaltungen und Erwartungen von Flüchtlingen in Österreich ( https://www.bmeia.gv.at/fileadmin/user upload/Zentr ale/Integration/; accessed 23-12-2018).

KommunalAkademie der Konrad-Adenauer-Stiftung e.V. (Hg.) (5. November 2015). Ausgewählte „Best-practice“Beispiele lokalen Engagements für Flüchtlinge. Integration und Abbau von Barrieren: Eine Momentaufnahme ersten Krisenmanagements. KonradAdenauer-Stiftung e.V. [https://ec.europa.eu/migrantintegration/?action=media.download\&uuid...0F00; accessed 03-12-2018].

Kurier (15-03-2018). Flüchtlinge. AMS sieht Integration unerwartet erfolgreich [https://kurier.at/politik/ausland/fluechtlinge-amssieht-integration-unerwartet-erfolgreich/313.953.563; accessed 20-12-2018]. 
Land Oberösterreich (2017): Grundversorgung von Fremden. (Asylwerber/innen). URL: https://www.landoberoesterreich.gv.at/26937.htm [Zugriff: 27.11.2017].

Lockwood, David (1964). Social integration and system integration. In: Zollschan, George K. und Hirsch, Walter (Hg.): Explorations in Social Change. London: 244-257.

Noschiel, Harald (18.05.2017). Freiwilligenarbeit als Baustein für Integration in Österreich. Eine Studie am Beispiel der „Initiative Langenzersdorf“, Masterarbeit, Zur Erlangung des akademischen Grades Master of Arts in Business, der Fachhochschule FH Campus Wien, Masterstudiengang: Public Management M17. [http://pub.fh-

campuswien.ac.at/obvfcwhsacc/download/pdf/206367 6?originalFilename=true ; accessed 04-12-2018].

OÖ Nachrichten (2018): Gekürzte Mindestsicherung verstößt nicht gegen EU-Recht. Online. 30.03.2018. URL: https://www.nachrichten.at/nachrichten/politik/landes politik/Gekuerzte-Mindestsicherung-verstoesst-nichtgegen-EU-Recht;art383,2526532 [Zugriff: 27.01.2018].

Ryan, Louise (2011). Migrants' social networks and weak ties: Accessing resources and constructing relationships post-migration, The Sociological Review, 59 (4), 707724.

Scholten, Peter, Fleur Baggereman, Linda Dellouche, Venja Kampen, Julia Wolf and Rick Ypma (August 2017). Policy Innovation in Refugee Integration? A comparative analysis of innovative policy strategies toward refugee 
integration in Europe. Faculteit der Sociale Wetenschappen

[https://www.rijksoverheid.nl/.../Policy+innovation+in+ refugee+integration.pd ; accessed 05-12-2018f].

[think difference:] Gesellschaftsanalyse. Innovation. Integration (Studienleitung: Dipl. Soz. wiss. Kenan Güngör) (Sept. 2006). Empirische Studie zu den integrationsrelevanten Handlungsfeldern, Aktivitäten und Perspektiven in den Gemeinden Oberösterreichs. Befragung der Bürgermeisterinnen und Bürgermeister inkl. Ergebnisdokumentation der Regionalveranstaltungsreihe in den Oö. Bezirken. BaselWien [http://www.think-difference.com/wpcontent/uploads/Gemeindestudie ENDF.pdf; accessed 18-12-2018].

Titelbach, Gerlinde, Thomas Davoine, Helmut Hofer, Philip Schuster, Mario Steiner (Juli/August 2013). Potentiale durch die Integration von Migrant/innen in Arbeitsmarkt und Bildung. Eine wirtschaftssoziologische Analyse struktureller Integration ÖIF-Forschungsbericht (https://www.integrationsfonds.at/publikationen/forsc hungsberichte/potentiale-durch-die-integration-vonmigrantinnen-in-arbeitsmarkt-und-bildung/; accessed 25-12-2018).

Wiedner, Jonas, Zerrin Salikutluk und Johannes Giesecke (März 2018). Arbeitsmarktintegration von Geflüchteten: Potenziale, Perspektiven und Herausforderungen, State-of-Research Papier 07, IMIS \& bicc

[https://flucht-forschung-transfer.de/wpcontent/uploads/2018/03/SoR-07- 
Arbeitsmarktintegration-von-Gefl\%C3\%BCchteten.pdf; accessed 25-12-2018].

WKO (21-03-2017). Arbeitsmarktintegration von Flüchtlingen

[https://www.wko.at/site/fachkraeftepotenzial/arbeits marktintegration.html; accessed 20-12-2018].

Wößmann, Ludger (2016), Bildung als Schlüssel zur Integration: Nur eine realistische Flüchtlingspolitik wird Erfolg haben, ifo Schnelldienst, 69 (1), 21-24.

Zschiedrich, Hilmar (2016): Wohnung verzweifelt gesucht. IN: Asyl Aktuell 3/2016. 2-8. URL: https://www.asyl.at/de/information/asylaktuell/2016/ [Zugriff: 27.01.2018].

\section{Quellenangaben}

Gemeindehomepage St. Andrä-Wördern 1:

https://www.staw.at/Unsere_Gemeinde/Unser_Ort/Zahle n_Fakten, abgerufen am 03.12.2018

Gemeindehomepage St. Andrä-Wördern 2:

https://www.staw.at/Politik Verwaltung/Wahlergebnisse als pdf (abgerufen am 03.12.2018)

URL 1:

https://www.meinbezirk.at/tulln/c-politik/waldhaeuslintegration-von-beginn-an-istgegenhausverstand_a2709251 (abgerufen am 23.11.2018) 


\section{ABKÜRZUNGSVERZEICHNIS DER INTERVIEWS}

I: NTERVIEWER/IN

R: RESPONDENT/IN

Die Quellenangaben für die Interviewzitate sind am Ende der Zitate mit folgenden Abkürzungen versehen:

\section{A für Afghanistan}

\section{I für Irak}

\section{S für Syrien}

\section{EXP Expert/inn/en}

m- männlich, w- weiblich

Danach wurde das Alter des/r Informanten/in zum Zeitpunkt des Interviews angegeben, die nachfolgenden Abkürzungen sind die Initialien der Interviewer/innen. 
Refugee Outreach

\& Research Network

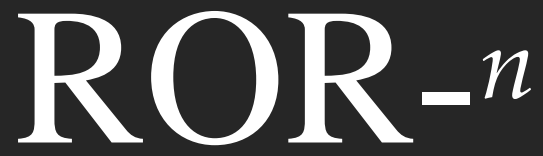

EIN WESENTLICHES ZIEL DES PROJEKTS LODA (LOSLASSEN-

DURCHSTEHEN - ANKOMMEN) LAG NEBEN DER AKQUISITION NEUER

WISSENSCHAFTLICHER DATEN ÜBER DIE NACH ÖSTERREICH

GEFLÜCHTETEN IN DER HANDLUNGSRELEVANTEN UMSETZUNG DER

RESULTATE UND IN WEITERER FOLGE IN DER FORMULIERUNG

KONKRETER HANDLUNGSEMPFEHLUNGEN.

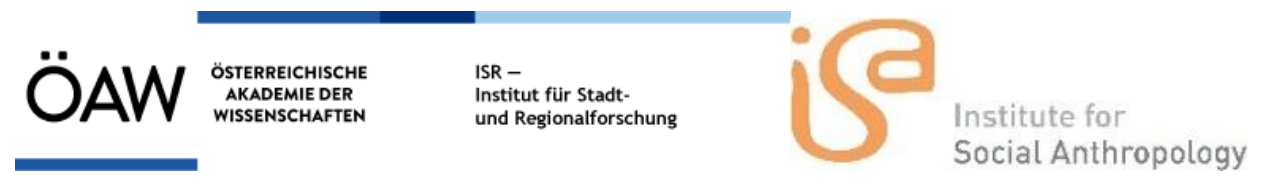

Institut für

KULTUR- UND SOZIALANTHROPOLOGII

Department of

SOCIAL AND CULTURAL ANTHROPOLOC

universität

wien 